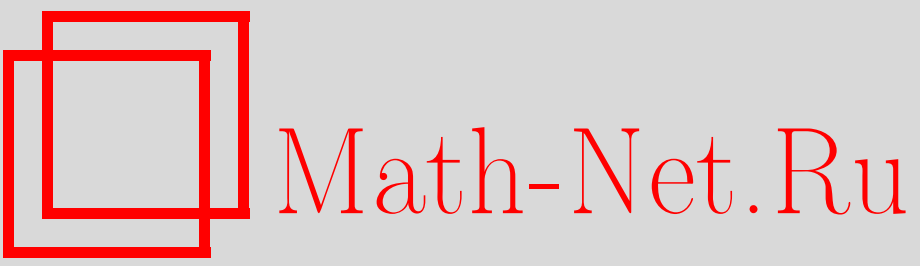

П. Е. Пушкарь, Ю. В. Чеканов, Комбинаторика фронтов лежандровых зацеплений и 4-гипотезы Арнольда, УМН, 2005, том 60, выпуск 1, 99-154

DOI: https://doi.org/10.4213/rm1390

Использование Общероссийского математического портала Math-Net.Ru подразумевает, что вы прочитали и согласны с пользовательским соглашением

http://www.mathnet.ru/rus/agreement

Параметры загрузки:

IP: 54.147 .182 .235

26 апреля 2023 г., 14:43:01 
УДК $514.7+515.16$

\title{
КОМБИНАТОРИКА ФРОНТОВ ЛЕЖАНДРОВЫХ ЗАЦЕПЛЕНИЙ И 4-ГИПОТЕЗЫ АРНОЛЬДА
}

\author{
П. Е. ПУШКАРЬ, Ю. В. ЧЕКАНОВ
}

\begin{abstract}
На выпуклой гладкой кривой на плоскости найдется по меньшей мере четьре точки, в которых ее кривизна достигает экстремума; кроме того, в случае кривой общего положения у нее найдется эквидистанта, имеющая по меньшей мере четыре точки возврата. В. И. Арнольд сформулировал на языке контактной топологии гипотезы о коориентированных фронтах на плоскости, обобщающие эти классические утверждения - гипотезу о четырех вершинах и гипотезу о четырех точках возврата. Настоящая статья посвящена доказательству этих гипотез и некоторых смежных утверждений. Основным ингредиентом доказательства, наряду с несложньм обобщением теории Штурма-Гурвица, является построенная в работе теория псевдоинволюций, доставляющая описание комбинаторной структуры фронтов на цилиндре. Обсуждается также связь теории псевдоинволюций с перестройками морсовских комплексов в однопараметрических семействах.

Библиографиия: 25 названий.
\end{abstract}

\section{СОДЕРЖАНИЕ}

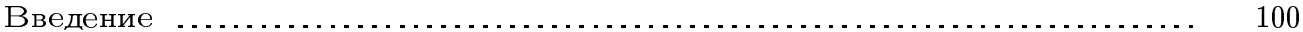

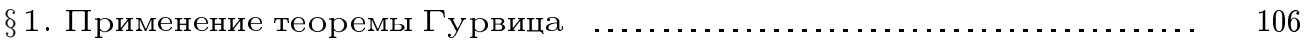

$\S 2$. Псевдоинволюции и теорема о продолжении $\ldots \ldots \ldots \ldots \ldots \ldots \ldots \ldots \ldots \ldots . . \ldots \ldots$

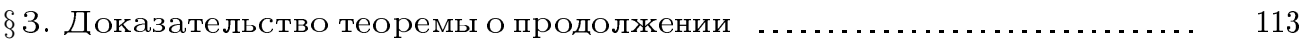

$\S 4$. Неоднозначность непрерывного продолжения и монодромия псевдо-

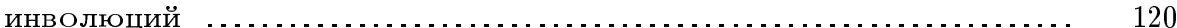

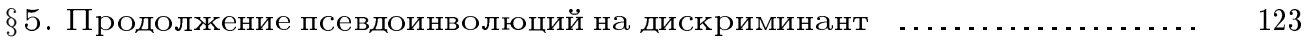

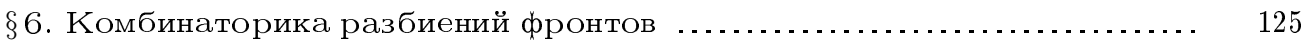

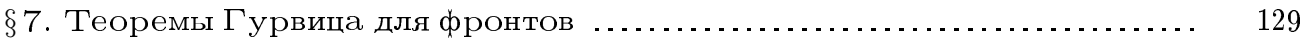

§. Доказательства обобщений теоремы Гурвица .................... 131

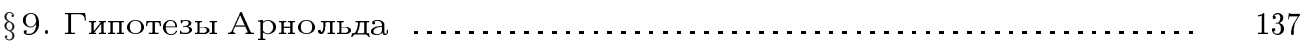

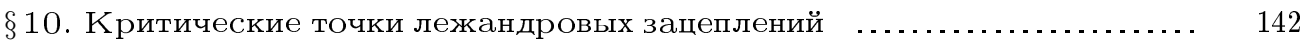

$\S 11$. Инварианты лежандровых зацеплений .......................... 145

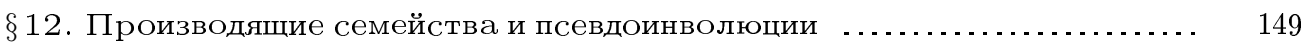

Список литературы . . . . . . . . . . . . . . . . . . 153

Работа выполнена при частичной поддержке Российского фонда фундаментальных исследований (грант № 02-01-00655). 


\section{Введение}

0.1. Контактные многообразия, лежандровы подмногообразия, фронты. Контактная форма на многообразии $V$ размерности $2 n+1$ - это такая 1 -форма $\alpha$, что форма $\alpha \wedge(d \alpha)^{n}$ нигде не обрашается в нуль (все встречающиеся в статье объекты предполагаются $C^{\infty}$-гладкими, если не оговорено противное). Контактнылм многообразием называется многообразие $V^{2 n+1}$, снабженное полем $\xi$ касательных гиперплоскостей $\xi_{x} \subset T_{x} V$, локально задаваемых как множества нулей контактной 1-формы: $\xi=\operatorname{ker} \alpha$. Если поле $\xi$ коориентируемо, то такая форма определена глобально. Подмногообразие $L$ размерности $n$ контактного многообразия $\left(V^{2 n+1}, \xi\right)$ называется лежандровым, если $T_{x} L \subset \xi_{x}$ для всех $x \in L$; при $\xi=\operatorname{ker} \alpha$ это условие эквивалентно условию $\left.\alpha\right|_{L} \equiv 0$.

Пусть $M$ - гладкое многообразие, тогда 1-струи функций на $M$ образуют многообразие $J^{1}(M)=T^{*} M \times \mathbb{R}$. Контактная структура на $J^{1}(M)$ задается естественной 1-формой $\alpha=d u-p d q$, где $u$ - координата на $\mathbb{R},(p, q)$ - канонические координаты Лиувилля на $T^{*} M$. Другой важньй пример контактного многообразия представляет собой сферизащия $S T^{*} N$ кокасательного расслоения гладкого многообразия $N$ - многообразие коориентированных касательных к $N$ гиперплоскостей (контактных элементов). Естественная коориентированная контактная структура на $S T^{*} N$ задается следуюшей конструкщией: коориентированная гиперплоскость в касательном пространстве к точке $X \in S T^{*} N$ есть прообраз под действием дифференциала проекции $\rho: S T^{*} N \rightarrow N$ коориентированной гиперплоскости $X \subset T N$.

Пусть $(V, \xi)$ - контактное многообразие. Гладкое расслоение $\tau:(V, \xi) \rightarrow B$ называется лежандровым, если его слои - лежандровы подмногообразия. Фронтом лежандрова подмногообразия $L \subset V$ назьвается его проекция $\tau(L) \subset B$. Всякая точка $x \in L$ определяет гиперплоскость $\tau_{*} \xi_{x} \subset T_{\tau(x)} B$, которая гладко зависит от точки $x$ и совпадает с касательной гиперплоскостью $T_{\tau(x)}(\tau(L))$ в гладких точках фронта, т.е. в точках, в которые лежандрово многообразие проецируется диффеоморфно. Будем называть эту гиперплоскость касательной гиперплоскостью к фронту $\tau(L)$. Естественные проекции $\rho: S T^{*} N \rightarrow N, \sigma: J^{1}(M) \rightarrow J^{0}(M)=M \times \mathbb{R}$ являются лежандровыми расслоениями. Гиперплоскости, касательные к фронтам проекции $\sigma$, невертикальны (не содержат направления $u$ ); гиперплоскости, касательные к фронтам проекции $\rho$, обладают естественной коориентацией, индуцированной коориентацией контактной плоскости. Всякое лежандрово многообразие общего положения в $J^{1}(M)$ (и, более того, всякое лежандрово многообразие, встречающееся в общем конечнопараметрическом семействе) восстанавливается по своему фронту; это же верно для лежандровых подмногообразий в $S T^{*} N$ и их коориентированных фронтов в $N$.

Предположим, что размерность контактного многообразия равна трем. Тогда фронт общего лежандрова зацепления (компактного лежандрова подмногообразия) есть кривая, особенности которой - точки трансверсального самопересечения и полукубические точки возврата (клювы). Клювы соответствуют точкам касания со слоем проекции. Всякая компактная кривая в $J^{0}(M), \operatorname{dim} M=1$, с такими особенностями, не имеющая вертикальных касательных, является фронтом лежандрова зацепления в $J^{1}(M)$; всякая коориентированная компактная кривая в $N, \operatorname{dim} N=2$, с такими особенностями является фронтом лежандрова зацепления в $S T^{*} N$. 
Одним из основных результатов настоящей работы является теорема 0.1 , сформулированная В.И. Арнольдом в качестве гипотезы [1]:

Теорема 0.1 (гипотеза Арнольда о четырех точках возврата). Пусть $\left\{L_{t \in[0,1]}\right\}-$ такой гладкий путь в пространстве лежандровых узлов в $S T^{*} \mathbb{R}^{2}$, что фронть $\rho\left(L_{0}\right), \rho\left(L_{1}\right)$ - это диффеоморфные окружности выпуклые кривые с противоположнными коориентациями. Тогда найдется такое $t_{0} \in[0,1]$, что лежсандров узел $L_{t_{0}}$ касается слоев проекчии $\rho$ по меньшей мере в четырех точках. Если семейство $\left\{L_{t}\right\}$ общего полохсения, то найдется такое $t_{0} \in[0,1]$, что фронт $\rho\left(L_{t_{0}}\right)$ имеет по меньшей мере четьре клюва.

0.2. Вершины. Для формулировки другой теоремы, также сформулированной В. И. Арнольдом в качестве гипотезы, нам понадобится понятие вершины лежсандровой кривой в $S T^{*} \mathbb{R}^{2}$. Дадим необходимые определения. Для лежандрова зацепления $L \subset S T^{*} \mathbb{R}^{2}$ определим обобшенное отображение кривизны $\operatorname{Curv}_{L}: L \rightarrow S^{1}=\mathbb{R P}^{1}$. Обозначим через $\xi$ векторное расслоение над $S T^{*} \mathbb{R}^{2}$, состояшее из лежаших в контактных плоскостях векторов, и рассмотрим его проективизацию $P \xi$. Пусть $z \in L$. Прямая $l_{z} \subset \xi_{z}$, касательная к $L$ в точке $z$, определяет элемент слоя над точкой $z$ расслоения $P \xi$. Мы построим тривиализашию расслоения $\xi$. Тем самым будет определена тривиализация расслоения $P \xi$ - отображение тотального пространства расслоения $P \xi$ в $\mathbb{R} P^{1}$. Отображение $\operatorname{Curv}_{L}: L \rightarrow \mathbb{R P}^{1}$ есть композиция отображения $z \mapsto l_{z}$ и этого тривиализуюшего отображения.

Для построения тривиализации расслоения $\xi$ зафиксируем на $\mathbb{R}^{2}$ евклидову структуру и ориентацию. Контактный элемент из $S T^{*} \mathbb{R}^{2}$ однозначно представим единичным ковектором $\cos q d x_{1}+\sin q d x_{2} \in T_{\left(x_{1}, x_{2}\right)}^{*} \mathbb{R}^{2}$, где $\left(x_{1}, x_{2}\right)$ - ориентированные евклидовы координаты на $\mathbb{R}^{2}, q \in S^{1}=\mathbb{R} / 2 \pi \mathbb{Z}$. Таким образом, $\left(x_{1}, x_{2}, q\right)$ - координаты на $S T^{*} \mathbb{R}^{2}$. Естественная контактная структура на $S T^{*} \mathbb{R}^{2}$ задается формой $\cos q d x_{1}+\sin q d x_{2}$. Векторные поля $v_{0}=\frac{\partial}{\partial q}, v_{1}=-\sin q \frac{\partial}{\partial x_{1}}+\cos q \frac{\partial}{\partial x_{2}}$ касаются контактного распределения и задают его тривиализацию. Пусть $z \in L, w=a_{0} v_{0}(z)+$ $a_{1} v_{1}(z) \in T_{z} L-$ ненулевой касательньй вектор. Представим $\mathbb{R} P^{1}$ как $\mathbb{R} \cup\{\infty\}$ и положим $\operatorname{Curv}_{L}(z)=\frac{a_{0}}{a_{1}}$, если $a_{1} \neq 0$, и $\operatorname{Curv}_{L}(z)=\infty$, если $a_{1}=0$.

(Отметим, что векторные поля $v_{0}$ и $v_{1}$ допускают следующее геометрическое описание. Поле $v_{0}$ всюду касается слоя расслоения $\rho: S T^{*} \mathbb{R}^{2} \rightarrow \mathbb{R}^{2}$ и отвечает врашению со скоростью 1 контактного элемента, приложенного в данной точке. Поле $v_{1}$ отвечает сдвигу со скоростью 1 контактного элемента вдоль прямой (геодезической), касающейся данного элемента. Это описание позволяет немедленно перенести конструкцию отображения $\operatorname{Curv}_{L}$ на случай лежандровых зацеплений в $S T^{*} M^{2}$, где $M^{2}$ - двумерное ориентированное риманово многообразие.)

Отображение $\operatorname{Curv}_{L}$ обобшает понятие кривизны плоской кривой в следующем смысле.

УТВЕРЖДЕНИЕ 0.2. Если L касается слоя проекции $\rho$ в точке $z$, mо $\operatorname{Curv}_{L}(z)$ $=\infty$, если не касается, то число $\operatorname{Curv}_{L}(z)$ с точностью до знака совпадает $c$ кривизной фронта $\rho(L)$ в точке $\rho(z)$.

Утверждение 0.2 доказьвается в $\S 1$. Назовем критические точки отображения $\operatorname{Curv}_{L}$ вершинами. Это определение согласовано с классическим определением вершин гладкой иммерсированной кривой в $\mathbb{R}^{2}$, если рассматривать эту кривую как 
фронт лежандрова многообразия. Для лежандрова зацепления $L$ общего положения проекции его вершин принадлежат гладкой части фронта $\rho(L)$.

Обозначим через $\mathscr{L}_{1}$ связную компоненту пространства лежандровых узлов в $S T^{*} \mathbb{R}^{2}$, содержашую узел, фронт которого есть окружность в $\mathbb{R}^{2}$. Следуюшая гипотеза была предложена В.И. Арнольдом (см. [4; с. 97], где она дана в иной, эквивалентной, формулировке).

Teоpema 0.3 (гипотеза Арнольда о четырех вершинах). Всякий лежандров узел $L \in \mathscr{L}_{1}$ имеет по меньшей мере четыре вершины.

0.3. Перестройки фронтов. Если $\left\{L_{t \in[0,1]}\right\}$ - общее однопараметрическое семейство иммерсированных лежандровых подмногообразий в $S T^{*} \mathbb{R}^{2}$, то фронты $\rho\left(L_{t}\right)$ лишь в конечное число моментов $t_{1}<\cdots<t_{k}$ имеют более сложные особенности, чем клювы и точки трансверсального пересечения двух ветвей. $\Phi$ ронты $\rho\left(L_{t}\right)$ остаются диффеоморфньми друг другу, пока $t$ меняется в пределах одного из промежутков $\left[0, t_{1}[,] t_{1}, t_{2}[, \ldots,] t_{k}, 1\right]$. При проходе параметра через значение $t_{i}$ фронты семейства $\left\{L_{t}\right\}$ перестраиваются одним из способов, показанных на рис. 1 (направление возрастания параметра может быть противоположно указанному стрелкой; коориентации ветвей для вариантов рис. $1 \mathrm{a}-\mathrm{c}$ могут быть любыми).

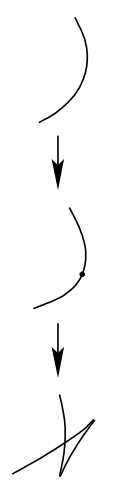

a

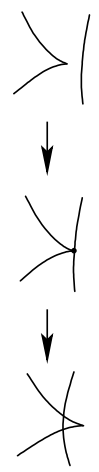

b

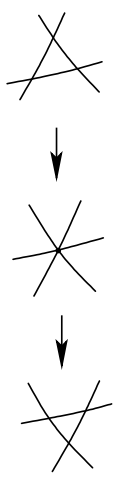

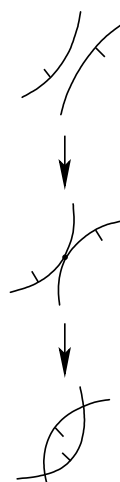

d

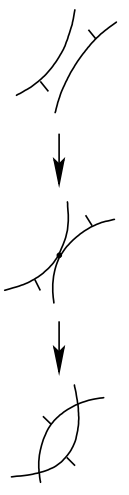

e

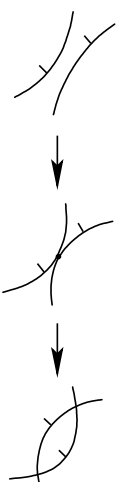

f

Рис. 1

Перестройка фронта, изображенная на рис. $1 \mathrm{f}$ (попутное самокасание), - единственная, для которой лежандрово многообразие $L_{t_{i}}$ имеет самопересечение. Утверждение теоремы 0.1 для семейств обшего положения имеет следуюшую эквивалентную форму: во всякой последовательности фронтов, получаюшихся друг из друга перестройками рис. $1 \mathrm{a}-\mathrm{f}$, начало и конец которой - вьпуклые кривые с противоположными коориентациями, найдется фронт с четырьмя клювами. Изучая связь этих перестроек с комбинаторикой фронтов, В.И. Арнольд построил теорию инвариантов конечного порядка для фронтов [2]. В. И. Арнольд обнаружил также, что утверждение теоремы 0.1 перестает быть верным, если разрешить лежандровым многообразиям семейства $\left\{L_{t}\right\}$ иметь самопересечения, т.е. если допустить перестройки попутного самокасания для фронтов [1]. Оказьвается, что и для теоремы 0.3 условие вложенности является сушественным: лежандров узел, фронт которого изображен на рис. 2 , 
имеет ровно две вершины и его можно соединить путем в классе лежандровых иммерсий с лежандровьм узлом из $\mathscr{L}_{1}$. Теорема 0.3 для случая выпуклых гладких кривых на плоскости - это классическая теорема о четырех вершинах (см. [7]). Для замкнутых вложенных плоских кривых ее доказал С. Мухопадхая [20]. Обобщение теоремы о четырех вершинах, рассматривающее точки уплощения пространственной кривой, лежащей на границе своей вьпуклой оболочки, получено В. Д. Седых [23].

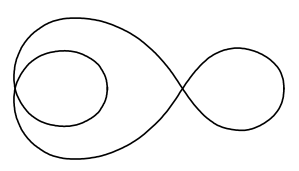

Рис. 2

Важньй пример семейства фронтов, для которого утверждение теоремы 0.1 следует из классической теоремы о четырех вершинах, - это эквидистанты выпуклой кривой. Изучение этого примера привело В. И. Арнольда к формулировке гипотезы о четырех точках возврата. Пусть $\mathrm{E}_{0}$ - вложенная коориентированная кривая на плоскости. Рассмотрим семейство эквидистант $\left\{\mathrm{E}_{t \in[0, T]}\right\}$, где кривая $\mathrm{E}_{t}$ получается из $\mathrm{E}_{0}$ сдвигом каждой точки вдоль положительной нормали на расстояние $t$, коориентируем $\mathrm{E}_{t}$ в том же направлении.

Для общей кривой $\mathrm{E}_{0}$ семейство эквидистант локально перестраивается как на рис. $1 \mathrm{a}-\mathrm{e}$. С физической точки зрения семейство эквидистант описьвает распространение волн в однородной изотропной среде. И для более обших распространений волн (неоднородных, неизотропных, неавтономных) перестройка попутного самокасания не может встретиться, так как волна не может догнать сама себя. Поэтому условие вложенности лежандровых подмногообразий в теореме 0.1 является естественным.

Вернемся к семейству эквидистант $\left\{\mathrm{E}_{t}\right\}$. Локальные особенности кривых $\mathrm{E}_{t}$ заметают каустику кривой $\mathrm{E}_{0}$ - кривую, составленную из центров кривизны. В центре кривизны, соответствуюшем вершине кривой $\mathrm{E}_{0}$, каустика имеет полукубическую особенность, а семейство фронтов $\left\{\mathrm{E}_{t}\right\}$ претерпевает перестройку, изображенную на рис. 1 a.

Пусть $\mathrm{E}_{0}$ - квадратично вьпуклая кривая общего положения, коориентированная внутрь. Радиус кривизны определяет гладкую функцию $R: \mathrm{E}_{0} \rightarrow \mathbb{R}$, критические точки которой - вершины кривой $\mathrm{E}_{0}$. Для обшего значения $t \in \mathbb{R}$ число клювов кривой $\mathrm{E}_{t}$ равно количеству точек кривой $\mathrm{E}_{0}$, в которых функция $R$ принимает значение $t$. В частности, для достаточно большого $T$ фронт $\mathrm{E}_{T}$ есть гладкая вьпуклая кривая, коориентированная наружу, а семейство лежандровых узлов с фронтами $\left\{\mathrm{E}_{t \in[0, T]}\right\}$ удовлетворяет (с точностью до замены параметра $t$ ) условию теоремы 0.1. Для этого семейства утверждение теоремы 0.1 следует из классической теоремы о четырех вершинах, так как всякая функция на окружности, имеюшая по крайней мере два минимума, принимает некоторое регулярное значение по меньшей мере четыре раза.

0.4. Замечательный контактоморфизм. Задание евклидовой структуры на $\mathbb{R}^{2}$ канонически определяет контактоморфизм между $S T^{*} \mathbb{R}^{2}$ и $J^{1}\left(S^{1}\right)$, где окружность $S^{1}$ отождествлена с единичной окружностью в $\mathbb{R}^{2}$. Опишем его. Контактньй элемент из $S T^{*} \mathbb{R}^{2}$ однозначно представим единичньм ковектором $\cos q d x_{1}+\sin q d x_{2} \in$ 
$T_{\left(x_{1}, x_{2}\right)}^{*} \mathbb{R}^{2}$, где $q \in S^{1}=\mathbb{R} / 2 \pi \mathbb{Z}$. Таким образом, $\left(x_{1}, x_{2}, q\right)$ - координаты на $S T^{*} \mathbb{R}^{2}$. Естественная контактная структура на $S T^{*} \mathbb{R}^{2}$ задается формой $\cos q d x_{1}+\sin q d x_{2}$. Отображение $S T^{*} \mathbb{R}^{2} \rightarrow J^{1}\left(S^{1}\right),\left(x_{1}, x_{2}, q\right) \mapsto(p, q, u)$, где $p=-x_{1} \sin q+x_{2} \cos q$, $u=x_{1} \cos q+x_{2} \sin q$, является контактоморфизмом, переводя форму $d u-p d q$ в форму $\cos q d x_{1}+\sin q d x_{2}$. Этот контактоморфизм изображен на рис. 3. Аналогичньм образом определяется контактоморфизм между $S T^{*} \mathbb{R}^{n+1}$ и $J^{1}\left(S^{n}\right)$ при произвольном $n$. Отождествим $S T^{*} \mathbb{R}^{2}$ и $J^{1}\left(S^{1}\right)$. Это отождествление играет важную роль в доказательстве теорем 0.1 и 0.3 . Основными объектами рассмотрения при этом являются фронты $\sigma(L) \subset J^{0}\left(S^{1}\right)$ лежандровых зацеплений $L \subset J^{1}\left(S^{1}\right)$.

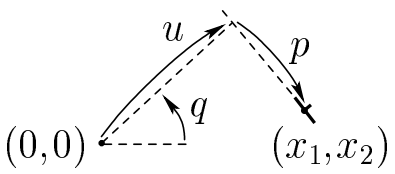

Рис. 3

0.5. Разбиения фронтов. Комбинаторньм инструментом, используемым в доказательстве теорем 0.1 и 0.3 , является теория псевдоинволюций фронтов в $J^{0}(M)$, где $\operatorname{dim} M=1$. Дадим некоторые определения этой теории для более простого случая $M=\mathbb{R}$ и сформулируем один из результатов, используя вместо языка псевдоинволюший эквивалентньй ему (при $M=\mathbb{R}$ ) язык правильных разбиений.

Пусть $\Sigma \subset J^{0}(\mathbb{R})$ - фронт общего лежандрова зацепления в $J^{1}(\mathbb{R})$. Кроме требования к особенностям фронта мы включаем в условие общего положения несовпадение $q$-координат точек самопересечения. Назовем сечением фронта $\Sigma$ его подмножество, являюшееся графиком такой непрерьвной функции $h:\left[q_{1}, q_{2}\right] \rightarrow \mathbb{R}$, что точки $\left(q_{1}, h\left(q_{1}\right)\right)$ и $\left(q_{2}, h\left(q_{2}\right)\right)$ - клювы фронта $\Sigma$.

Неупорядоченньй набор $D=\left\{\Gamma_{1}, \ldots, \Gamma_{N}\right\}$ сечений фронта $\Sigma$ называется разбиением фронта $\Sigma$, если $\Sigma=\bigcup_{i=1}^{N} \Gamma_{i}$ и любые два сечения пересекаются лишь в конечном числе точек. Назовем точку $x$ самопересечения фронта $\Sigma$ переключающей для разбиения $D$, если сечения из $D$, содержашие точку $x$, имеют в этой точке излом. Отметим, что всякое разбиение $D$ однозначно восстанавливается по своему набору $\operatorname{Sw}(D)$ переключающих точек. Назовем разбиение $\left\{\Gamma_{1}, \ldots, \Gamma_{N}\right\}$ фронта $\Sigma$ правильныцм, если сечения $\Gamma_{i}$ можно разбить на пары так, что сечения из одной пары пересекаются в своих концах и только в них. Это разбиение на пары определяет свободную инволюцию $\theta_{D}$ на множестве $D$. Отметим, что не всякий фронт допускает правильное разбиение. Например, правильного разбиения нет у фронта, содержашего фрагмент "зигзаг": 乙 (не пересекаюшийся с другими ветвями фронта).

Правильные разбиения были впервые рассмотрены Я.М.Элиашбергом [12]. Настояшая работа во многом явилась результатом обдумьвания конструкций из [12] .

0.6. Положительность. Пусть $D$-правильное разбиение, $x=\left(q_{0}, u_{0}\right) \in \operatorname{Sw}(D)$. Обозначим через $\Gamma$ и $\Gamma^{\prime}$ сечения из $D$, проходящие через точку $x$. Сечения $\Gamma, \Gamma^{\prime}, \theta_{D}(\Gamma)$, $\theta_{D}\left(\Gamma^{\prime}\right)$ попарно различны, так как разбиение $D$ правильное. Рассмотрим прямую $l_{q_{1}}=\left\{q=q_{1}\right\}$, где точка $q_{1} \neq q_{0}$ близка к $q_{0}$. Обозначим $z_{1}=\Gamma \cap l_{q_{1}}, z_{2}=\Gamma^{\prime} \cap l_{q_{1}}$, $z_{1}^{\prime}=\theta_{D}(\Gamma) \cap l_{q_{1}}, z_{2}^{\prime}=\theta_{D}\left(\Gamma^{\prime}\right) \cap l_{q_{1}}$. 
Назовем переключаюшую точку $x$ положительной (для разбиения $D$ ), если выполнено следующее условие неперемежаемости: либо отрезки $\left[z_{1}, z_{1}^{\prime}\right]$ и $\left[z_{2}, z_{2}^{\prime}\right]$ не пересекаются, либо один есть подмножество другого. Это определение не зависит от выбора точки $q_{1}$

Правильное разбиение $D$ фронта $\Sigma$ назьвается положительным, если все его переключаюшие точки положительны.

ТЕОРема 0.4. Пусть $L$ и $L^{\prime}$ - общие лежандровь зацепления в $J^{1}(\mathbb{R})$, гомотопные в классе лежандровых зачеплений. Тогда число положстельных разбиений для фронтов $\sigma(L)$ и $\sigma\left(L^{\prime}\right)$ одинаково.

Интересно отметить, что понятие положительного правильного разбиения было независимо введено Д. Б. Фуксом [16]. В [16] изучалась связь между существованием положительного разбиения фронта лежандрова узла $L \subset J^{1}(\mathbb{R})$ и аугментациями дифференциальной градуированной алгебры, ассоциированной с $L$ (определение дифференциальной градуированной алгебры и аугментащии см. в [11]).

0.7. План статьи. В $\S 1$ приведено доказательство теоремы 0.1 для частного случая, иллюстрируюшее идеи полного доказательства, связанные с применением теоремы Гурвица. В $\S 2$ даны основные определения теории псевдоинволюций и сформулирован ее главный результат - теорема 2.5 о продолжении положительных псевдоинволюций на общие однопараметрические семейства фронтов. Теорема 2.5 доказана в $\S 3$, где такое продолжение строится явно. В $\S 4$ изучается (не)однозначность продолжения положительных псевдоинволюций и монодромия, возникающая при продолжении вдоль петель. Продолжение псевдоинволюций на необшие фронты изучается в 55 . В $\S 6$ доказаны некоторые результаты, связанные с комбинаторикой разбиений фронтов. В $\S 7$ сформулированы обобшения теоремы Гурвица, в которой вместо функции на окружности участвует фронт лежандрова подмногообразия пространства $J^{1}\left(S^{1}\right)$. Их доказательства содержатся в $\S 8$. Гипотезы Арнольда о клювах и вершинах доказаны в $\S 9$ (с использованием результатов из предыдуших параграфов), там же приведены некоторые их обобшения и другие результаты о клювах и вершинах фронтов. Критические точки лежандровых зацеплений (и их связь с теоремой Гурвица) обсуждается в $\S 10$. Теория псевдоинволюций позволяет определить инварианты лежандровых узлов. Полученные таким образом инварианты описаны в $\S 11$. В $\S 12$ объясняется связь теории производяших семейств лежандровых многообразий и теории псевдоинволюший. В частности, показано, каким образом производящее семейство определенного вида определяет псевдоинволюцию на фронте задаваемого им лежандрова подмногообразия пространства $J^{1}\left(S^{1}\right)$.

Мы выражаем глубокую признательность нашему учителю В.И. Арнольду, открьвшему, среди многого другого, замечательную связь между дифференциальной геометрией и контактной топологией. Мы очень благодарны ему за поддержку и настойчивость, без которых эта статья не была бы закончена. Мы благодарны Ф. Аикарди, О. Я. Виро, В.В.Горюнову, М. Л. Концевичу, М. Ф. Прохоровой, В. Д. Седых, Э. Феррану, Д.Б. Фуксу, Я.М. Элиашбергу за стимулирующий интерес к работе и полезные обсуждения. 


\section{$\S 1$ Применение теоремы Гурвица}

1.1. Кривизна и 1-формы. Дадим описание отображения $\operatorname{Curv}_{L}$ в терминах дифференщиальных форм. Рассмотрим 1-формы $\omega=d q, \beta=-\sin q d x_{1}+\cos q d x_{2}$ на $S T^{*} \mathbb{R}^{2}$. Они удовлетворяют соотношениям $\left\langle\omega, v_{0}\right\rangle=\left\langle\beta, v_{1}\right\rangle=1,\left\langle\omega, v_{1}\right\rangle=$ $\left\langle\beta, v_{0}\right\rangle=0$, где $v_{0}, v_{1}$ - векторные поля, участвуюшие в определении отображения $\operatorname{Curv}_{L}$ в п. 0.2. Поэтому для любой точки $z \in L$ и любого ненулевого касательного вектора $w \in T_{z} L$ выполняется $\operatorname{Curv}_{L}(z)=\langle\omega(z), w\rangle:\langle\beta(z), w\rangle$.

1.2. Доказательство утверждения 0.2. Если $L$ касается слоя проекции $\rho$, то $\langle\beta(z), w\rangle=0$ и утверждение доказано. Пусть $L$ не касается слоя проекции $\rho$ в точке $z$. Выберем такую локальную параметризашию $t \mapsto\left(x_{1}(t), x_{2}(t), q(t)\right)$, $0 \mapsto z$ зацепления $L$, что $\dot{x}_{1}^{2}+\dot{x}_{2}^{2} \equiv 1$, т.е. отображение $t \mapsto\left(x_{1}(t), x_{2}(t)\right)$ есть натуральная параметризация фронта $\rho(L)$. Кривизна фронта $\rho(L)$ в точке $\rho(z)$ по определению равна $\sqrt{\ddot{x}_{1}^{2}(0)+\ddot{x}_{2}^{2}(0)}$. Проверим, что это число совпадает с $|\dot{q}(0)|$. Из условия контактности $\dot{x}_{1} \cos q+\dot{x}_{2} \sin q=0$ и условия $\dot{x}_{1}^{2}+\dot{x}_{2}^{2} \equiv 1$ вытекает, что $\left(\dot{x}_{1}(t), \dot{x}_{2}(t)\right)= \pm(\sin (q(t)),-\cos (q(t)))$ при всех $t$. Следовательно, $\left(\ddot{x}_{1}(t), \ddot{x}_{2}(t)\right)= \pm(\cos (q(t)), \sin (q(t))) \dot{q}(t)$ и потому $\sqrt{\ddot{x}_{1}^{2}(0)+\ddot{x}_{2}^{2}(0)}=|\dot{q}(0)|$. Поскольку $\left\langle\omega(z),\left(\dot{x}_{1}(0), \dot{x}_{2}(0), \dot{q}(0)\right)\right\rangle=\dot{q}(0)$ и $\left\langle\beta(z),\left(\dot{x}_{1}(0), \dot{x}_{2}(0), \dot{q}(0)\right)\right\rangle=-\dot{x}_{1}(0) \sin (q(0))+$ $\dot{x}_{2}(0) \cos (q(0))= \pm 1$ (так как единичные векторы $(\cos (q(0)), \sin (q(0)))$ и $\left(\dot{x}_{1}(0), \dot{x}_{2}(0)\right)$ ортогональны), получаем $\operatorname{Curv}_{L}(z)= \pm \dot{q}(0)$.

1.3. Кривизна и дифференциальный оператор Штурма. Пусть $L \subset S T * \mathbb{R}^{2}$ $=J^{1}\left(S^{1}\right)$ - лежандрово зацепление. Нам понадобится описание в терминах фронта $\sigma(L)$ множества точек, в которых $L$ касается слоев проекции $\rho$. Обозначим через $G_{L}$ множество неособых точек проекции $\left.\sigma\right|_{L}$. Определим отображение $F_{L}: L \rightarrow \mathbb{R} \mathrm{P}^{1}=$ $\mathbb{R} \cup\{\infty\}$. Положим $F_{L}(y)=\infty$, если $y \notin G_{L}$. В окрестности точки $y \in G_{L}$ лежандрово зацепление $L$ совпадает с 1-графиком $j^{1} h$ некоторой функции $h: U \rightarrow \mathbb{R}, U \subset S^{1}$, т.е. задается уравнениями $u=h(q), p=h^{\prime}(q)$, где $q \in U$. Рассмотрим дифференциальньй оператор Штурма $D_{1} h=d^{2} h / d q^{2}+h$ и положим $F_{L}(y)=D_{1} h\left(q_{y}\right)$, где $q_{y}-$ $q$-координата точки $y$.

Лемма 1.1. Отображения $F_{L}$ и $1 / \operatorname{Curv}_{L}$ совпадают (полагаем $1 / 0=\infty$, $1 / \infty=0)$. Точка $z$-особая точка проекиии $\left.\rho\right|_{L}$, если и только если $z \in G_{L}$ $u F_{L}(z)=0$.

ДоКАЗАТЕЛЬСТВО. Если $z \in L$ - особая точка проекции $\left.\sigma\right|_{L}$, то $F_{L}(z)=\infty$, а $\operatorname{Curv}_{L}(z)=0$, так как $\left.\beta\right|_{L}(z)=\left.d q\right|_{L}(z)=0$. Пусть $z \in G_{L}, L$ совпадает с $j^{1} h$ вблизи $z$. Тогда $1 / \operatorname{Curv}_{L}(z)=\langle\beta(z), v\rangle /\langle\omega(z), v\rangle$. Вектор $\zeta=\frac{\partial}{\partial q}+h^{\prime}\left(q_{z}\right) \frac{\partial}{\partial u}+h^{\prime \prime}\left(q_{z}\right) \frac{\partial}{\partial p}$, где $q_{z}-q$-координата точки $z$, касается $L$ в точке $z$. Поскольку $\beta=-\sin q d x_{1}+$ $\cos q d x_{2}=d p+u d q$, получаем $1 / \operatorname{Curv}_{L}(z)=\langle\beta(z), \zeta\rangle /\langle\omega(z), \zeta\rangle=h^{\prime \prime}\left(q_{z}\right)+h\left(q_{z}\right)=$ $F_{L}(z)$. Второе утверждение леммы следует из того, что равенство $\operatorname{Curv}_{L}(z)=\infty$ равносильно $\left.\beta\right|_{L}(z)=0$, а $\left.\beta\right|_{L}(z)=0$ тогда и только тогда, когда вектор $v_{0}(z)$ касается $L$.

1.4. Теорема Гурвица и гладкие фронты. При доказательстве теоремы 0.1 можно считать, что начало координат на плоскости лежит внутри (квадратично выпуклых) фронтов $\rho\left(L_{0}\right), \rho\left(L_{1}\right)$. Из этого вытекает, что фронт $\sigma\left(L_{0}\right)$ (соответственно $\left.\sigma\left(L_{1}\right)\right)$ является графиком отрицательной (соответственно положительной) гладкой 
функции на $S^{1}$. Заметим, что если $\left\{\rho\left(L_{t}\right)\right\}$ - семейство эквидистант, то фронты $\sigma\left(L_{t}\right)$ получаются из фронта $\sigma\left(L_{0}\right)$ сдвигом вдоль координаты $u$.

Предположим, что фронты $\sigma\left(L_{t}\right)$ являются графиками гладких функций $H_{t}$. Следующее доказательство теоремы 0.1 для этого частного случая принадлежит В. И. Арнольду [1]. Пусть $t_{0} \in[0,1]$ таково, что $\int_{S^{1}} H_{t_{0}}(q) d q=0$. Коэффициенты при нулевой и первых гармониках ряда Фурье функции $D_{1} H_{t_{0}}$ равны нулю. По теореме Гурвица [19] функция $D_{1} H_{t_{0}}$ обрашается в нуль по меньшей мере четыре раза. Поскольку нули функции $D_{1} H_{t_{0}}$ соответствуют нулям отображения $F_{L_{t_{0}}}$, это доказывает утверждение теоремы 0.1 для такого семейства.

1.5. Теорема Гурвица и фронты с особенностями. Основная идея доказательства теоремы 0.1 заключается в переносе этого рассуждения на случай произвольного семейства $\left\{L_{t}\right\}$ лежандровых узлов. Мы построим такое непрерывное семейство $\left\{H_{t \in[0,1]}\right\}$ непрерьвных функций на $S^{1}$, что для любого $t$ график функции $H_{t}$ является подмножеством фронта $\sigma\left(L_{t}\right)$. Будет доказано, что если $\int_{S^{1}} H_{t_{0}}(q) d q=0$, то $F_{L_{t_{0}}}$ имеет по меньшей мере четыре нуля.

В качестве иллюстрации рассмотрим лежандров узел $L$, фронт $\Sigma=\sigma(L)$ которого имеет ту же комбинаторную структуру, что и фронт, изображенный на рис. 4 . Покажем, опуская детали, что если $\int_{S^{1}} H(q) d q=0$, где $H$ - непрерывная функция на $S^{1}$, график которой есть подмножество фронта $\Sigma$, то $L$ касается слоев проекции $\rho$ по меньшей мере в четырех точках.

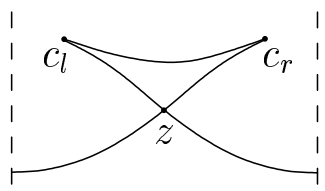

Рис. 4

Предположим для простоты, что функция $F_{L}$ отлична от нуля в обоих прообразах точки самопересечения $z=\left(q_{0}, u_{0}\right)$ фронта $\Sigma$. Функцию $D_{1} H$ можно рассматривать как обобшенную функцию на $S^{1}$ вида $C \delta_{q_{0}}+g$, где $\delta_{q_{0}}-\delta$-функция с носителем в точке $q_{0}, g$ - непрерывная функция на $S^{1} \backslash\left\{q_{0}\right\}$, имеюшая ненулевые пределы слева и справа в точке $q_{0}$. Из рис. 4 видно, что $C<0$. Обобщенная функция $D_{1} H$ аннулирует тригонометрические многочлены степени $\leqslant 1$. Отсюда несложно вывести, модифицируя стандартное доказательство теоремы Гурвица, что $D_{1} H$ меняет знак по меньшей мере четыре раза. Мы считаем, что знак обобшенной функции $D_{1} H$ в точке $q_{0}$ отрицателен (равен знаку числа $C$ ), и говорим, что $D_{1} H$ меняет знак справа (слева) в точке $q_{0}$, если правый (левый) предел функции $g$ в точке $q_{0}$ положителен.

Если все четыре смены знака происходят вне точки $q_{0}$, то утверждение доказано. Если перемены знака (одна или две) приходятся на точку $q_{0}$, то мы будем искать недостающие нули отображения $F_{L}$ на прообразах в $L$ сторон $\left[z, c_{l}\right],\left[z, c_{r}\right]$ криволинейного треугольника с вершинами $z, c_{r}, c_{l}$. Докажем, что если $D_{1} H$ меняет знак справа (слева) в точке $q_{0}$, то $F_{L}$ обрашается в нуль в одной из точек прообраза стороны $\left[z, c_{l}\right]\left(\left[z, c_{r}\right]\right)$. В самом деле, отрезок $\left[z, c_{l}\right] \subset \Sigma$ есть график некоторой функции $h:\left[q_{l}, q_{0}\right] \rightarrow \mathbb{R}$, где $c_{l}=\left(q_{l}, u_{l}\right)$. Функция $h$ является гладкой всюду, кроме точки $q_{l}$, 
и $h^{\prime \prime}$ стремится к $-\infty$ при $q \rightarrow q_{l}$. Следовательно, найдется такая точка $q \in\left[q_{l}, q_{0}\right]$, что $D_{1} h(q)=0$. Для левой перемены знака утверждение аналогично. Рассуждение закончено.

Доказательство теоремы 0.1 для произвольных фронтов проходит по той же схеме. При этом строится некоторое разбиение фронта на части (обобщающее разбиение рассмотренного вьше фронта $\Sigma$ на четыре части). При построении этого разбиения используется теория псевдоинволюций.

1.6. План доказательства теоремы 0.3. Мы показьваем, что фронт $\sigma(L)$ всякого лежандрова узла $L \in \mathscr{L}_{1}$ содержит непрерывное сечение $\left\{u=H_{L}(q)\right\}$ расслоения $J^{0}\left(S^{1}\right) \rightarrow S^{1}$, непрерьвно зависящее от $L$. Сдвиг лежандрова зацепления $L$ (и фронта $\sigma(L))$ вдоль $u$ переводит вершины в вершины. Доказательство теоремы 0.3 использует следуюший трюк: сдвигая $L$ вдоль направления $u$, мы добиваемся того, что $\int_{S^{1}} H_{L}(q) d q=0$. Используя те же рассуждения, что и в доказательстве теоремы 0.1 , указьваем четыре точки, в которых $F_{L}$ обрашается в нуль. Вершины $L$ - это критические точки отображения $F_{L}$. Мы докажем (используя аналог леммы Ролля для отображений в окружность), что между любыми двумя соседними нулями из этих четырех найдется вершина лежандрова узла $L$. Это завершит доказательство.

\section{§2. Псевдоинволюции и теорема о продолжении}

2.1. Особенности фронтов. Пусть $M=S^{1}$, или $M=\mathbb{R}$, или $M=I$, где $I-$ отрезок в $\mathbb{R}$ или $S^{1}$. В случае, когда $M=I$, мы будем называть лежсандровьлм зацеплением в $J^{1}(M)$ компактное лежандрово подмногообразие $L$ с краем такое, что $\partial L=\partial J^{1}(M) \cap L$ (т.е. проекция $J^{1}(I) \rightarrow I$ отображает край $L$ в концы отрезка $\left.I\right)$, $L$ трансверсально $\partial J^{1}(I)$ во всех точках своего края, любые две точки края $L$, проецируюшиеся в один конец отрезка $I$, имеют различные $u$-координаты. Обозначим через $\pi$ проекцию $J^{0}(M) \rightarrow M,(q, u) \mapsto q$, через $\sigma$ - проекцию $J^{1}(M) \rightarrow J^{0}(M)$. Пусть $\Sigma=\sigma(L) \subset J^{0}(M)$ - фронт лежандрова зацепления $L \subset J^{1}(M)$. Обозначим через $G_{\Sigma}$ множество неособых точек фронта $\Sigma$ (в том числе проекции краевых точек $L$ ), через $X_{\Sigma}$ множество трансверсальных двойных самопересечений, через $C_{\Sigma}$ множество невырожденных клювов (точек, в окрестности которых фронт диффеоморфен полукубической параболе), через $Z_{\Sigma}=\Sigma \backslash\left(G_{\Sigma} \cup X_{\Sigma} \cup C_{\Sigma}\right)$ множество точек, в которых фронт имеет более сложную особенность. В случае, когда $M=I$, такую точку $x \in \Sigma$, что $\pi(x) \in \partial I$, мы относим к $G_{\Sigma}$.

В пространстве $\mathscr{L}$ всех лежандровых зацеплений в $J^{1}(M)$ рассмотрим две (особые) гиперповерхности - $\mathscr{S}$, состоящую из таких зацеплений $L$, что $Z_{\sigma(L)}$ непусто (т.е. у фронта $\sigma(L)$ есть сложные особенности), и $\mathscr{E}$, состоящую из таких $L$, для которых найдутся две особые точки фронта $\sigma(L)$ с совпадаюшими проекциями на $M$. Назовем гиперповерхность $\mathscr{D}=\mathscr{S} \cup \mathscr{E}$ дискриминантом. Назовем лежандрово зацепление $L \subset J^{1}(M)$ (и его фронт $\left.\sigma(L)\right) \sigma$-общим, если $L \in \mathscr{L} \backslash \mathscr{D}$.

2.2. Определение псевдоинволюции. Непрерывное отображение $\mathrm{P}: G_{\Sigma} \cup C_{\Sigma}$ $\rightarrow \Sigma$ назьвается псевдоинволюиией фронта $\Sigma \subset J^{0}(M)$, если оно удовлетворяет следуюшим четырем условиям:

(PI1) $\left.\pi\right|_{\Sigma}=\left.\pi\right|_{\Sigma} \circ \mathrm{P}$

$(\mathrm{PI}) \mathrm{P}^{2}(x)=x$ при $\mathrm{P}(x) \in G_{\Sigma} \cup C_{\Sigma}$; 
(PI3) $\mathrm{P}(x)=x$, если и только если $x \in C_{\Sigma}$;

(PI4) для любой точки $x \in X_{\Sigma}$ найдется такая окрестность $U \subset J^{0}(M)$, что $\mathrm{P}\left(U \cap G_{\Sigma}\right)$ не пересекается с $U$.

2.3. Псевдоинволюции и разбиения. Пусть $\Sigma \subset J^{0}(M)$ - фронт лежандрова зацепления, $\Lambda$ - отрезок или окружность, $\gamma: \Lambda \rightarrow \Sigma$-такое непрерьвное отображение, что: (1) композиция $\gamma$ и проекции $J^{0}(M) \rightarrow M$ локально является вложением; (2) неособые точки фронта $\Sigma$ имеют не более одного прообраза под действием $\gamma ;(3)$ если $\Lambda$ - отрезок, то каждый из его концов $\gamma$ переводит либо в клюв фронта $\Sigma$, либо в точку края $\partial J^{0}(M)=\pi^{-1}(\partial M)$. Такое отображение $\gamma$, рассматриваемое с точностью до гомеоморфизмов $\Lambda$, будем называть сечением фронта $\Sigma$. Если $M=\mathbb{R}$ или $M=I$, то сечение $\gamma$ однозначно описьвается своим образом $\Gamma=\gamma(\Lambda)$, которьй мы также будем называть сечением (и использовать наравне с $\gamma$ ). Таким образом, это определение согласовано с определением, данным во введении для случая $M=\mathbb{R}$.

Пусть $\Sigma \subset J^{0}(M)$ - $\sigma$-общий фронт. Разбиением фронта $\Sigma$ назьвается такое множество $D$ сечений фронта $\Sigma$, что каждая неособая точка фронта $\Sigma$ принадлежит образу ровно одного сечения из $D$. Пусть $x \in X_{\Sigma}$, тогда найдутся такие сечения $\gamma_{i}: \Lambda_{i} \rightarrow \Sigma$ $(i \in\{1,2\})$ из $D$ и точки $s_{i} \in \Lambda_{i}$, что $\gamma_{i}\left(s_{i}\right)=x$ (если $\gamma_{1}=\gamma_{2}$, то $\left.s_{1} \neq s_{2}\right)$. Назовем точку $x$ переключающей для разбиения $D$, если образы под действием $\gamma_{1}, \gamma_{2}$ малых окрестностей точек $s_{1}, s_{2}$ имеют излом в точке $x$, и непереключающей, если онигладки. Множество переключаюших точек разбиения $D$ обозначим через $\mathrm{Sw}(D)$. Отображение $D \mapsto \mathrm{Sw}(D)$ задает взаимно однозначное соответствие между множеством разбиений фронта $\Sigma$ и множеством подмножеств множества $X_{\Sigma}$.

УТВЕРЖДЕНИЕ 2.1. Всякая псевдоинволюиия $\mathrm{P}$ б-общего фронта $\Sigma \subset J^{0}(M)$ однозначно определяет разбиение $D_{\mathrm{P}}$ фронта $\Sigma$ и свободную инволючию $\theta_{\mathrm{P}}: D_{\mathrm{P}} \rightarrow D_{\mathrm{P}}$ такие, что для любого сечения $\gamma: \Lambda \rightarrow \Sigma$ из $D_{\mathrm{P}}$ и любой точки $x \in \gamma(\Lambda) \cap\left(G_{\Sigma} \cup C_{\Sigma}\right)$ точка $\mathrm{P}(x)$ принадлежит образу сечения $\theta_{\mathrm{P}}(\gamma)$.

ДоКАЗАТЕЛЬСТВо. При помощи разбиения $M$ на части утверждение сводится к случаю, когда $M=I$ и фронт $\Sigma$ имеет ровно одну особую точку (если все точки фронта $\Sigma$ неособы, утверждение очевидно). Пусть $x$ - точка самопересечения фронта $\Sigma$. Неособые компоненты фронта $\Sigma$ переводятся псевдоинволюцией Р в сечения фронта $\Sigma$. Два из этих сечений, $\Gamma_{1}$ и $\Gamma_{2}$, проходят через точку $x$ (иначе псевдоинволюция Р переставляла бы точки, близкие к $x$, в противоречие условию (PI4)). Сечения разбиения $D_{\mathrm{P}}$ в этом случае - это $\Gamma_{1}, \Gamma_{2}$ и неособые ветви фронта. Ясно, что требуемая инволюция $\theta_{\mathrm{P}}$ сушествует и единственна. Случай, когда единственная особая точка $\Sigma$ есть клюв, очевиден.

Элементы разбиения $D_{\mathrm{P}}$ будем назьвать $\mathrm{P}$-сечениям $и$ фронта $\Sigma$. Точку $x \in X_{\Sigma}$ назовем переключающей для псевдоинволющии $\mathrm{P}$, если она является переключающей для разбиения $D_{\mathrm{P}}$, и непереключающей в противном случае. Множество переключающих точек псевдоинволюции $\mathrm{P}$ обозначим через $\mathrm{Sw}(\mathrm{P})$.

Пусть $\Sigma \subset J^{0}(M)$ - $\sigma$-общий фронт, $M=\mathbb{R}$ или $M=I$. Назовем пару $(D, \theta)$, где $D$ - разбиение фронта $\Sigma, \theta: D \rightarrow D$ - свободная инволюция, правильной, если для любого сечения $\Gamma \in D$ вьполняется $\pi(\Gamma)=\pi(\theta(\Gamma))$ и точки пересечения сечений $Г$ и $\theta(\Gamma)$ - это клювы фронта $\Sigma$. 
УТВЕРЖДЕНИЕ 2.2. Если $M=\mathbb{R}$ или $M=I$, то отображсение $\mathrm{P} \mapsto\left(D_{\mathrm{P}}, \theta_{\mathrm{P}}\right)$ является взаимно однозначным соответствием между множеством псевдоинволющий б-общего фронта $\Sigma$ и множеством правильных пар для фронта $\Sigma$.

ДокАЗАТЕЛЬСТво. Если $\mathrm{P}$ - псевдоинволюция фронта $\Sigma$, то пара $\left(D_{\mathrm{P}}, \theta_{\mathrm{P}}\right)$ является правильной по построению, данному в доказательстве утверждения 2.1. Если $(D, \theta)$ - правильная пара, то, согласно определению отображения $\mathrm{P} \mapsto\left(D_{\mathrm{P}}, \theta_{\mathrm{P}}\right)$, данному в формулировке утверждения 2.1 , найдется ровно одна такая псевдоинволюция $\mathrm{P}$, что $\left(D_{\mathrm{P}}, \theta_{\mathrm{P}}\right)=(D, \theta)$.

Отметим, что при $M=\mathbb{R}$ для всякого правильного разбиения $D$ найдется ровно одна такая инволюция $\theta: D \rightarrow D$, что пара $(D, \theta)$ правильная. Поэтому из утверждения 2.2 вытекает, что псевдоинволющии $\sigma$-общего фронта $\Sigma \subset J^{0}(\mathbb{R})$ находятся во взаимно однозначном соответствии с правильными разбиениями фронта $\Sigma$.

Конечность числа псевдоинволюций вытекает из следующего утверждения.

УТВЕРЖДЕНИЕ 2.3. Пусть $\Sigma \subset J^{0}(M)-\sigma$-общий фронт, точка $q_{0} \in M$ такова, что множество $\Sigma^{q_{0}}=\Sigma \cap \pi^{-1}\left(q_{0}\right)$ не содержит особьх точек фронта. Пусть $\mathrm{P}, \mathrm{P}^{\prime}$ - такие псевдоинволюции фронта $\Sigma$, ито $\mathrm{Sw}(\mathrm{P})=\mathrm{Sw}\left(\mathrm{P}^{\prime}\right)$ и обе они одинаково действуют на множсестве $\Sigma^{q_{0}}$. Тогда $\mathrm{P}=\mathrm{P}^{\prime}$.

ДокАЗАТЕльство. Утверждение достаточно доказать для случая $M=I$ (действительно, если $\mathrm{P} \neq \mathrm{P}^{\prime}$, то различаются и ограничения псевдоинволюций на некоторый $\sigma$-общий фронт $\Sigma^{I}=\Sigma \cap \pi^{-1}(I) \subset J^{0}(I)$, где $I$ - отрезок в $M$, содержащий точку $\left.q_{0}\right)$. Из того, что $\mathrm{Sw}(\mathrm{P})=\mathrm{Sw}\left(\mathrm{P}^{\prime}\right)$, следует, что разбиения $D_{\mathrm{P}}$ и $D_{\mathrm{P}^{\prime}}$ совпадают. Остается показать, что $\theta_{\mathrm{P}}(\Gamma)=\theta_{\mathrm{P}^{\prime}}(\Gamma)$ для любого сечения $\Gamma \in D_{\mathrm{P}}$. Пусть $Г$ пересекает $\Sigma^{q_{0}} \mathrm{~B}$ точке $y$. Тогда каждое из сечений $\theta_{\mathrm{P}}(\Gamma), \theta_{\mathrm{P}^{\prime}}(\Gamma)$ содержит точку $\mathrm{P}(y)=\mathrm{P}^{\prime}(y)$, и потому $\theta_{\mathrm{P}}(\Gamma)=\theta_{\mathrm{P}^{\prime}}(\Gamma)$. Если $\Gamma$ не пересекает $\Sigma^{q_{0}}$, то $\Gamma$ содержит клюв. Обозначим через $\Gamma_{1}$ другое сечение из $D_{\mathrm{P}}=D_{\mathrm{P}^{\prime}}$, содержашее этот клюв. Тогда каждое из сечений $\theta_{\mathrm{P}}(\Gamma), \theta_{\mathrm{P}^{\prime}}(\Gamma)$ также содержит этот клюв, и потому $\theta_{\mathrm{P}}(\Gamma)=\theta_{\mathrm{P}^{\prime}}(\Gamma)$.

2.4. Положительность. Пусть $\mathrm{P}$ - псевдоинволюция фронта $\Sigma \in J^{0}(M), x=$ $\left(q_{0}, u_{0}\right) \in \mathrm{Sw}(\mathrm{P})$. Рассмотрим прямую $\left\{q=q_{1}\right\} \subset J^{0}(M)$, где точка $q_{1} \neq q_{0}$ близка к $q_{0}$. Обозначим через $z_{1}, z_{2}$ точки из $\Sigma \cap l$, близкие к точке $x$. Назовем точку $x$ положительной (по отношению к $\mathrm{P}$ ), если вьполнено следуюшее условие неперемежаемости: либо отрезки $\left[z_{1}, \mathrm{P}\left(z_{1}\right)\right]$ и $\left[z_{2}, \mathrm{P}\left(z_{2}\right)\right]$ не пересекаются, либо один есть подмножество другого. Не положительную переключающую точку назовем отрицательной. Это определение не зависит от выбора точки $q_{1}$ и согласовано с определением для случая $M=\mathbb{R}$, данным во введении.

Псевдоинволюция Р назьвается поло жительной, если все ее переключаюшие точки положительны.

2.5. Масловский потенциал. Пусть $l:[0,1] \rightarrow L$ - гладкий путь общего положения с концами в множестве $G_{L}$ неособых точек проекции $L \rightarrow M$. Индекс Маслова $m(l) \in \mathbb{Z}$ этого пути в случае зацепления обшего положения есть число клювов на пути $l$, подсчитанных со знаками, определенными следующим образом. Если точка $\sigma(l(t)) \in \sigma(L)$ проходит через клюв фронта $\sigma(L)$ с нижней ветви на верхнюю, то знак равен +1 , а если с верхней на нижнюю, то -1 . Если зацепление $L$ необщее, то $m(l)$ определяется как индекс Маслова близкого пути на близком зацеплении общего 
положения (результат не зависит от выбора шевеления). Числом Маслова $m(L)$ лежандрова узла назьвается модуль индекса Маслова пути, обходщего узел один раз. Число Маслова $m(L)$ всегда четно. Действительно, его четность совпадает с четностью числа клювов фронта $\sigma(L)$ (мы предполагаем, что $L$ - лежандров узел обшего положения). При обходе $L$ прообразы правых $(\succ)$ и левых $(\prec)$ клювов фронта $\sigma(L)$ чередуются. Поэтому правых клювов столько же, сколько левых, и их обшее число четно. Число Маслова лежандрова зацепления $L$ - это наибольший общий делитель чисел Маслова для компонент $L$, диффеоморфных окружности.

Пусть $m \in \mathbb{Z}$ - неотрицательный делитель числа $m(L)$. Масловский потенциал зацепления $L$ - это такая локально постоянная функщия $\mu: G_{L} \rightarrow \mathbb{Z} / m \mathbb{Z}$, что для любой пары точек $z_{1}, z_{2} \in G_{L}$, лежаших на одной компоненте зацепления $L$, разность $\mu\left(z_{2}\right)-\mu\left(z_{1}\right)$ равна индексу Маслова пути, соединяющего $z_{1}$ с $z_{2}$ (приведенного по модулю $m$ ). Все масловские потенциалы со значением в $\mathbb{Z} / m \mathbb{Z}$ получаются из одного прибавлением функции, постоянной на каждой из компонент зацепления $L$.

2.6. Масловская псевдоинволюция. Точка самопересечения $x$ фронта $\sigma(L)$ называется масловской относительно масловского потенциала $\mu$ зацепления $L$, если $\mu(z)=\mu\left(z^{\prime}\right)$, где $z$ и $z^{\prime}-$ различные точки $L$, проектирующиеся в $x$. Разбиение $\sigma$-обшего фронта назьвается масловским относительно масловского потенциала $\mu$, если все его переключаюшие точки масловские относительно $\mu$. Заметим, что если зацепление $L$ связно, то для данного $m$ разбиение фронта $\sigma(L)$ является или не является масловским независимо от выбора потенциала $\mu: G_{L} \rightarrow \mathbb{Z} / m \mathbb{Z}$.

Псевдоинволюция $\mathrm{P} \sigma$-общего фронта $\sigma(L)$ назьвается масловской относительно $\mu$, если для любой такой пары неособых точек $y, y^{\prime}$ фронта $\Sigma$, что $\mathrm{P}(y)=y^{\prime}$ и точка $y^{\prime}$ лежит вьше точки $y$ (т.е. ее $u$-координата больше), для прообраза $z$ точки $y$ и прообраза $z^{\prime}$ точки $y^{\prime}$ в $L$ вьполнено условие $\mu\left(z^{\prime}\right)=\mu(z)+1$. Назовем сечение $\gamma: \Lambda \rightarrow \Sigma$ фронта $\Sigma \subset J^{0}(M)$ длинным, если либо $M=S^{1}$ и $\Lambda$ - окружность, либо $M=I$ и $\gamma$ отображает оба конца отрезка $\Lambda$ в край $\partial J^{0}(M)$. При проверке того, что псевдоинволюция является масловской, удобно пользоваться следующим утверждением.

УТВЕРЖДЕНИЕ 2.4. Пусть $\mu$-масловский потенциал на б-общем зацеплении $L \subset J^{1}(M), \mathrm{P}-$ псевдоинволючия фронта $\Sigma=\sigma(L)$. Псевдоинволюиия $\mathrm{P}$ является масловской относительно $\mu$ тогда и только тогда, когда разбиение $D_{\mathrm{P}}$ масловское относительно $\mu$ и для всякого длинного Р-сечения найдется такая пара $y, y^{\prime}$ неособых точек фронта $\Sigma$, одна из которых принадлежит образу этого сечения, что $\mathrm{P}(y)=y^{\prime}$, точка $y^{\prime}$ лежит выше точки $y$, для прообраза z точки у и прообраза $z^{\prime}$ точки $y^{\prime}$ в $L$ выполнено условие $\mu\left(z^{\prime}\right)=\mu(z)+1$.

ДокАЗАТЕльство. Пусть псевдоинволюция Р масловская. Докажем, что всякая переключающая точка $x \in \mathrm{Sw}(\mathrm{P})$ масловская. Рассмотрим такую точку $z \in L$, что $\mathrm{P}(\sigma(z))=x$. Пусть $z^{\prime}, z^{\prime \prime}-$ точки на $L$, лежашие в различных малых полуокрестностях точки $z$. Точки $\mathrm{P}\left(\sigma\left(z^{\prime}\right)\right), \mathrm{P}\left(\sigma\left(z^{\prime \prime}\right)\right)$ принадлежат различным гладким ветвям фронта $\Sigma$, пересекаюшимся в точке $z$. Значения масловского потенциала $\mu$ в точках $z^{\prime}, z^{\prime \prime}$ совпадают, поэтому совпадают его значения в точках $L$, являюшихся прообразами точек $\mathrm{P}\left(\sigma\left(z^{\prime}\right)\right), \mathrm{P}\left(\sigma\left(z^{\prime \prime}\right)\right)$. Следовательно, совпадают значения $\mu$ в прообразах точки $x$, т.е. точка $x$ масловская. Таким образом, разбиение $D_{\mathrm{P}}$ масловское; вторая половина условия выполнена автоматически. 
Докажем обратное утверждение. Так как разбиение $D_{\mathrm{P}}$ масловское, то для всякого Р-сечения $\gamma$ масловский потенциал $\mu$ принимает одинаковое значение на прообразах в $L$ всех неособых точек фронта $\Sigma$, принадлежаших образу сечения $\gamma$. Поэтому для доказательства масловости Р достаточно проверить, что для всякого Р-сечения найдется такая пара $y, y^{\prime}$ неособых точек фронта $\Sigma$, одна из которых принадлежит образу этого сечения, что $\mathrm{P}(y)=y^{\prime}$, точка $y^{\prime}$ лежит выше точки $y$, для прообраза $z$ точки $y$ и прообраза $z^{\prime}$ точки $y^{\prime}$ в $L$ вьполнено условие $\mu\left(z^{\prime}\right)=\mu(z)+1$. Для длинных сечений это условие вьполнено по предположению. Образ всякого короткого (не длинного) Р-сечения $\gamma$ содержит клюв. Рассмотрим пару точек $y, y^{\prime} \in \Sigma$ с совпадающей $q$-координатой, близких к этому клюву; одна из них лежит в образе сечения $\gamma$. Из определения масловского потенциала следует, что сформулированное вьше условие вьполнено для пары точек $y, y^{\prime}$.

2.7. Семейства псевдоинволюций и теорема о продолжении. Гладкое однопараметрическое семейство $\left\{L_{t \in[a, b]}\right\}$ лежандровых зацеплений (и семейство их фронтов) назьвается $\sigma$-общ, $и$, если лежандровы зацепления $L_{a}, L_{b} \sigma$-общие, а путь $t \mapsto L_{t}$ трансверсально пересекает гиперповерхности $\mathscr{S}, \mathscr{E}$ в их гладких точках и не пересекает $\mathscr{S} \cap \mathscr{E}$.

Рассмотрим семейство $\left\{\mathrm{P}_{t \in[a, b]}\right\}$ псевдоинволюций, где $\mathrm{P}_{t}-$ псевдоинволюция фронта $\sigma\left(L_{t}\right)$. Семейство $\left\{\mathrm{P}_{t}\right\}$ назьвается непрерывныл, если отображение $(t, x) \mapsto\left(t, \mathrm{P}_{t}(x)\right)$, определенное на множестве

$$
\left\{(t, x) \mid x \in G_{\sigma\left(L_{t}\right)} \cup C_{\sigma\left(L_{t}\right)}\right\} \subset[a, b] \times J^{0}(M)
$$

непрерьвно. Семейство псевдоинволюций $\left\{\mathrm{P}_{t}\right\}$ называется положительным, если псевдоинволюции $\mathrm{P}_{t}$ положительны при всех таких $t$, что зацепление $L_{t} \sigma$-общее.

Пусть $D$ - разбиение $\sigma$-общего фрронта $\Sigma$. Назовем его эйлеровой характеристикой число

$$
\chi(D)=\frac{1}{2} \#\left(C_{\Sigma}\right)-\#(\mathrm{Sw}(D)) .
$$

Эйлеровой характеристикой $\chi(\mathrm{P})$ псевдоинволюции $\mathrm{P}$ назовем эйлерову характеристику разбиения $D_{\mathrm{P}}$. Семейство $\left\{\mathrm{P}_{t}\right\}$ назьвается характеристическим, если эйлеровы характеристики $\chi\left(\mathrm{P}_{t}\right)$ совпадают при всех таких $t$, что зацепление $L_{t} \sigma$-общее.

Пусть на $L_{a}$ задан масловский потенциал $\mu_{a}$. Тогда он однозначно продолжается до непрерьвного (в очевидном смысле) семейства $\left\{\mu_{t \in[a, b]}\right\}$ масловских потенциалов на зацеплениях семейства $\left\{L_{t \in[a, b]}\right\}$. Семейство псевдоинволюций $\left\{\mathrm{P}_{t}\right\}$ назьвается масловским по отношению к масловскому потенциалу $\mu_{a}$ (и к семейству масловских потенциалов $\left.\left\{\mu_{t}\right\}\right)$, если псевдоинволюция $\mathrm{P}_{t}$ масловская относительно $\mu_{t}$ при всех таких $t$, что $L_{t}-\sigma$-общее зацепление.

Tеорема 2.5 (теорема о продолжении). Пусть $M=S^{1}, M=\mathbb{R}$ или $M=I$, $\left\{L_{t \in[a, b]}\right\}-\sigma$-общее семейство лежандровых зацеплений в $J^{1}(M)$. Каждая положительная псевдоинволюиия $\mathrm{P}_{a}$ фронта $\sigma\left(L_{a}\right)$ единственным образом продолжается до непрерывного положительного характеристического семейства $\left\{\mathrm{P}_{t \in[a, b]}\right\}$ псевдоинволюиий. 
Eсли, кроме того, псевдоинволюиия $\mathrm{P}_{a}$ масловская по отношению кмасловскому потенииалу $\mu_{a}$, то семейство $\left\{\mathrm{P}_{t}\right\}$ также масловское по отношению $\kappa \mu_{a}$.

Определения непрерьвного, положительного, характеристического семейства псевдоинволюций выдерживают смену направления параметра $t$. Из этого вытекает

УТВЕРЖДЕНИЕ 2.6. В условиях теоремы 2.5 отображение $\mathrm{P}_{a} \mapsto \mathrm{P}_{b}$ является взаимно однозначным соответствием между множсествами положительных псевдоинволючий для фронтов $\sigma\left(L_{a}\right)$ и $\sigma\left(L_{b}\right)$, сохраняющим эйлеровы характеристики.

Теорема 0.4 является немедленным следствием утверждения 2.6.

\section{§. Доказательство теоремы о продолжении}

3.1. Расслоенные диффеоморфизмы и псевдоинволюции. Обозначим через $\mathrm{G}_{M}$ группу, состояшую из диффеоморфизмов $J^{0}(M)$, расслоенных над $M$. Расслоенный диффеоморфизм $g \in \mathrm{G}_{M}$ взаимно однозначно отображает псевдоинволюции фронта $\Sigma \subset J^{0}(M)$ в псевдоинволюции фронта $g(\Sigma)$, при этом положительные псевдоинволюции переходят в положительные. Следуюшая лемма немедленно следует из определений.

Лемма 3.1. Пусть $\left\{\Sigma_{t \in[a, b]}\right\}-\sigma$-общее семейство фронтов в $J^{0}(M),\left\{g_{t \in[a, b]}\right\}$ - гладкое семейство әлементов группь $\mathrm{G}_{M}, g_{a}=\mathrm{id}$. Тогда $\left\{g_{t}\right\}$ взаимно однозначно отображает непрерывные семейства псевдоинволючий для семейства фронтов $\left\{\Sigma_{t}\right\}$ в непрерывные семейства псевдоинволюций для семейства фронтов $\left\{g_{t}\left(\Sigma_{t}\right)\right\}$. При этом положительные семейства переходят в положительные, характеристические в характеристические, масловские относительно $\mu_{a}$ в масловские относительно $\mu_{a}$.

Если семейство зацеплений $\left\{L_{t}\right\}$ в теореме 2.5 не пересекает дискриминант $\mathscr{D}$, то лемма 3.1 сводит утверждение теоремы к случаю постоянного семейства $\left(L_{t} \equiv L_{a}\right)$, для которого утверждение теоремы очевидно.

3.2. Типичные перестройки. Если $\left\{L_{t}\right\}-\sigma$-общее семейство зацеплений, $L_{c} \in \mathscr{D}$, то фронты $\Sigma_{t}=\sigma\left(L_{t}\right)$ при проходе параметра $t$ через значение $c$ могут перестраиваться (с точностью до действия однопараметрических семейств расслоенных диффеоморфизмов) как показано на рис. 5 (локальные перестройки - пересечения с $\mathscr{S}$ ) и рис. 6 (нелокальные перестройки - пересечения с $\mathscr{E}$ ). Нарисованы только компоненты, участвуюшие в перестройке; отметим, что перестройка III с точностью до симметрий совпадает с обратной к ней перестройкой $\mathrm{III}^{-1}$, перестройка $\mathrm{XX}-$ с перестройкой $\mathrm{XX}^{-1}$, перестройка $\mathrm{CC}_{+}-$с перестройкой $\mathrm{CC}_{+}^{-1}$.

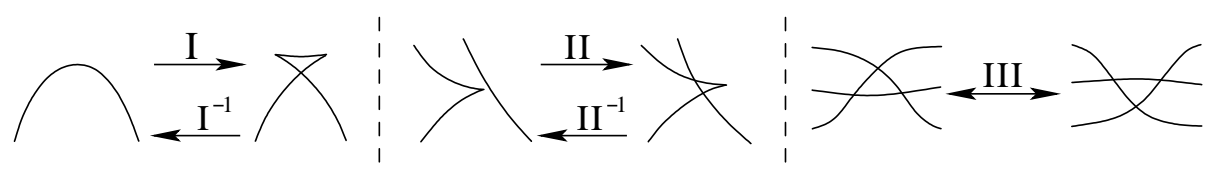

Рис. 5 


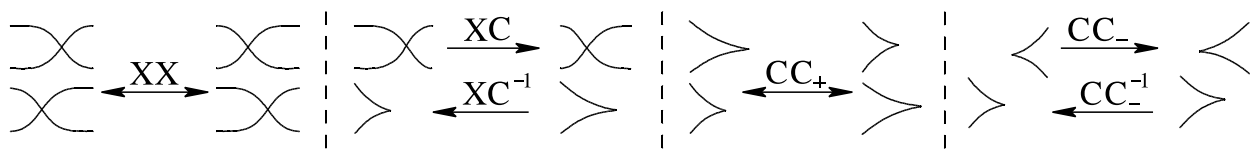

Рис. 6

3.3. Простые и полупростые семейства фронтов. Назовем $\sigma$-общее семейство лежандровых зацеплений $\left\{L_{t \in[a, b]}\right\}$ в $J^{1}(I)$ (и семейство фронтов $\left\{\Sigma_{t}=\sigma\left(L_{t}\right)\right\}$ ) простылм, если "край" $\partial \Sigma_{t}=\Sigma_{t} \cap \partial\left(J^{0}(I)\right)$ не зависит от $t, L_{t} \in \mathscr{D}$ ровно для одного значения параметра $t=c \in] a, b\left[\right.$, фронт $\Sigma_{c}$ содержит ровно одну особую точку в случае локальной перестройки $\left(L_{c} \in \mathscr{S}\right)$ и ровно две особые точки с совпадаюшими $q$-координатамив случае нелокальной перестройки $\left(L_{c} \in \mathscr{E}\right)$. Назовем $\sigma$-общее семейство лежандровых зацеплений $\left\{L_{t \in[a, b]}\right\}$ в $J^{1}(M)$ (и семейство фронтов $\left\{\Sigma_{t}=\sigma\left(L_{t}\right)\right\}$ ) полупростылм по отношению к отрезку $I \subset M$, если фронты $\Sigma_{t}$ совпадают вне $J^{0}(I)$ при всех $t$ и семейство $\left\{\Sigma_{t}^{I}=\Sigma_{t} \cap J^{0}(I)\right\}$ фронтов в $J^{0}(I)$ просто. Разбивая отрезок $[a, b]$ на части и применяя лемму 3.1 , мы получаем, что утверждение теоремы 2.5 достаточно доказать для полупростых семейств.

Покажем, что можно ограничиться рассмотрением простых семейств. Действительно, пусть семейство $\left\{\Sigma_{t}\right\}$ полупросто по отношению к отрезку $I, \mathrm{P}_{a}-$ псевдоинволюция фронта $\Sigma_{a}$. Тогда всякое непрерывное семейство псевдоинволюций $\left\{\mathrm{P}_{t}^{I}\right\}$ для фронтов $\Sigma_{t}^{I}$ такое, что $\mathrm{P}_{a}^{I}$ есть ограничение $\mathrm{P}_{a}$, однозначно продолжается до непрерьвного семейства псевдоинволюций $\left\{\mathrm{P}_{t}\right\}$ для фронтов $\Sigma_{t}$, при этом $\mathrm{P}_{t}$ совпадает с $\mathrm{P}_{a}$ вне $J^{0}(I)$ при всех $t$. Очевидно, что семейство $\left\{\mathrm{P}_{t}\right\}$ положительное, если $\left\{\mathrm{P}_{t}^{I}\right\}$ положительное, характеристическое, если $\left\{\mathrm{P}_{t}^{I}\right\}$ характеристическое, и масловское, если $\left\{\mathrm{P}_{t}^{I}\right\}$ масловское.

Лемма 3.2. Пусть $\left\{\Sigma_{t \in[a, b]}\right\}$ - простое семейство фронтов в $J^{0}(I), \mathrm{P}_{a}$ и $\mathrm{P}_{b}-$ псевдоинволюиии фронтов $\Sigma_{a}$ и $\Sigma_{b}$, совпадающие на $\partial \Sigma_{a}=\partial \Sigma_{b}$. Тогда $\mathrm{P}_{a}$ и $\mathrm{P}_{b}$ включаются в единственное непрерывное семейство псевдоинволюций $\left\{\mathrm{P}_{t \in[a, b]}\right\}$.

Если $\mathrm{P}_{a}$ и $\mathrm{P}_{b}$ положительны, то семейство $\left\{\mathrm{P}_{t}\right\}$ положительное. Если $\chi\left(\mathrm{P}_{a}\right)=\chi\left(\mathrm{P}_{b}\right)$, то семейство $\left\{\mathrm{P}_{t}\right\}$ характеристическое. Eсли $\left\{\mu_{t}\right\}-$ непрерьвное семейство масловских потенциалов, псевдоинволючия $\mathrm{P}_{a}$ масловская относительно $\mu_{a}$ и все переключающие точки псевдоинволюиии $\mathrm{P}_{b}$ масловские относительно $\mu_{b}$, то семейство $\left\{\mathrm{P}_{t}\right\}$ масловское.

ДОКАЗАТЕЛЬСТВО. При Помоши леммы 3.1 псевдоинволюция $\mathrm{P}_{a}$ единственньм образом включается в непрерывное семейство псевдоинволюций $\left\{\mathrm{P}_{t}\right\}$, где $t \in[a, c[$, и псевдоинволюция $\mathrm{P}_{b}$ единственным образом включается в непрерьвное семейство псевдоинволюций $\left\{\mathrm{P}_{t}\right\}$, где $\left.\left.t \in\right] c, b\right]$.

Построим отображение $\mathrm{P}_{c}$. Обозначим через $q_{0} \in I$ проекцию особых точек фронта $\Sigma_{c}$. Пусть $x \in \Sigma_{c}, \pi(x) \neq q_{0}$. Рассмотрим отрезок $I^{\prime} \subset I \backslash\left\{q_{0}\right\}$, содержаший один из концов $q^{\prime}$ отрезка $I$ и некоторую окрестность точки $\pi(x)$. Найдутся такие $a_{0} \in[a, c[$, $\left.\left.b_{0} \in\right] c, b\right]$, что у фронта $\Sigma_{t}^{I^{\prime}}$ нет особых точек при $t \in\left[a_{0}, b_{0}\right]$. Поскольку все псевдоинволюции $\mathrm{P}_{t}$ при $t \in\left[a_{0}, b_{0}\right] \backslash\{c\}$ одинаково действуют на $\Sigma_{t}^{q^{\prime}}=\pi^{-1}\left(q^{\prime}\right) \cap \Sigma_{t}$, по лемме 3.1 существует и единственно такое непрерывное семейство $\left\{\mathrm{P}_{t \in\left[a_{0}, b_{0}\right]}^{\prime}\right\}$ псевдо- 
инволюций фронтов $\Sigma_{t}^{I^{\prime}}$, что $\mathrm{P}_{t}^{\prime}(y)=\mathrm{P}_{t}(y)$ при $t \in\left[a_{0}, b_{0}\right] \backslash\{c\}, y \in \Sigma_{t}^{I^{\prime}}$. Положим $\mathrm{P}_{c}(x)=\mathrm{P}_{c}^{\prime}(x)$. Отображение $(t, y) \mapsto\left(t, \mathrm{P}_{t}(y)\right)$ непрерывно в точке $(c, x)$.

Пусть $x$ - неособая точка фронта $\Sigma_{c}, \pi(x)=q_{0}$. Так как семейство фронтов $\left\{\Sigma_{t}\right\}$ просто, то существует и единственно непрерьвное семейство сечений $\Gamma_{t \in[a, b]}$, состоящих из неособых точек фронта $\Sigma_{t}$, такое, что $x \in \Gamma_{c}$. Отображение $(t, y) \mapsto\left(t, \mathrm{P}_{t}(y)\right)$, уже определенное при $y \in \Gamma_{t}, t \in[a, b],(t, y) \neq(c, x)$, однозначно непрерьвно продолжается в точку $(c, x)$, так как функции $f_{t}: I \rightarrow \mathbb{R}, t \in[a, b] \backslash\{c\}$, графиками которых являются сечения $\mathrm{P}_{t}\left(\Gamma_{t}\right)$, равномерно липшищевы и сходятся при $t \rightarrow c$ равномерно вне любой окрестности точки $q_{0}$.

Если $x$ - клюв фронта $\Sigma_{c}$, то полагаем $\mathrm{P}_{c}(x)=x$. Непрерьвность отображения $(t, y) \mapsto\left(t, \mathrm{P}_{t}(y)\right)$ в точке $(c, x)$ следует из того, что ветви фронта, входяшие в клюв, переставляются псевдоинволюцией. Непрерьвность семейства $\left\{\mathrm{P}_{t \in[a, b]}\right\}$ доказана. То, что $\mathrm{P}_{c}-$ псевдоинволюция фронта $\Sigma_{c}$, легко следует из непрерьвности.

Пусть псевдоинволюция $\mathrm{P}_{a}$ масловская относительно $\mu_{a}$ и все переключающие точки псевдоинволюции $\mathrm{P}_{b}$ масловские относительно $\mu_{b}$. Для всякого длинного $\mathrm{P}_{b}$-сечения можно выбрать такую пару точек $y, y^{\prime} \in \partial \Sigma_{b}$, одна из которых принадлежит образу этого сечения, что $\mathrm{P}_{b}(y)=y^{\prime}$ и точка $y^{\prime}$ лежит вьше точки $y$. Тогда $\mathrm{P}_{a}(y)=y^{\prime}$ по условию леммы. Рассмотрим такие точки $z, z^{\prime} \in \partial L_{b}$, что $\sigma(z)=y, \sigma\left(z^{\prime}\right)=y^{\prime}$. Из масловости псевдоинволюции $\mathrm{P}_{a}$ получаем $\mu_{a}\left(z^{\prime}\right)=\mu_{a}(z)+1$. Из непрерьвности семейства $\left\{\mu_{t}\right\}$ следует, что ограничения $\mu_{a}$ и $\mu_{b}$ на $\partial L_{b}=\partial L_{a}$ совпадают. Значит, $\mu_{b}\left(z^{\prime}\right)=\mu_{b}(z)+1$. Применяя утверждение 2.4 , получаем, что псевдоинволюция $\mathrm{P}_{b}$ масловская относительно $\mu_{b}$. Оставшиеся утверждения леммы теперь очевидны.

Окончание доказательства теоремы проводится отдельно для каждой из перестроек фронтов.

3.4. Перестройка I. Пусть при $t=c$ происходит перестройка I. Точка $x_{b}$ самопересечения фронта $\Sigma_{b}$ является переключающей для любой псевдоинволюции фронта $\Sigma_{b}$. Это следует из того, что $C^{1}$-гладкое сечение фронта $\Sigma_{b}$, соединяющее два клюва, может отображаться псевдоинволюцией $\mathrm{P}_{b}$ только в сечение, имеющее излом в точке $x_{b}$. Сушествует ровно одна псевдоинволюция $\mathrm{P}_{b}$ фронта $\Sigma_{b}$, совпадаюшая с $\mathrm{P}_{a}$ на $\partial \Sigma_{b}=\partial \Sigma_{a}$, при этом $\chi\left(\Sigma_{b}\right)=\chi\left(\Sigma_{a}\right)=0$. Положительность псевдоинволюции $\mathrm{P}_{b}$ вытекает из того, что между сечениями фронта $\Sigma_{b}$, соединяющими два клюва, нет других точек фронта $\Sigma_{b}$. Точка $x_{b}$ масловская для любого выбора масловского потенциала. Применение леммы 3.2 завершает доказательство теоремы для случая перестройки I.

3.5. Перестройка $\mathrm{I}^{-1}$. Точка самопересечения фронта $\Sigma_{a}-$ переключающая для любой псевдоинволюции. Дальнейшие рассуждения аналогичны рассуждениям для перестройки I.

3.6. Перестройка II. Пусть $\mathrm{P}_{b}$ - положительная псевдоинволюция фронта $\Sigma_{b}$, совпадаюшая с $\mathrm{P}_{a}$ на $\partial \Sigma_{b}=\partial \Sigma_{a}$. Применяя к фронту $\Sigma_{b}$ следуюшую лемму, мы получаем, что такая псевдоинволющия $\mathrm{P}_{b}$ единственна и $\mathrm{Sw}\left(\mathrm{P}_{b}\right)=\varnothing$.

Лемма 3.3. Если фронт $\Sigma$ имеет фрагмент, изображсенный на рис. 7 справа (не пересекающийся с остальными частями фронта), то для любой положительной псевдоинволючии $\mathrm{P}$ точки х и у не являются переключающими. 

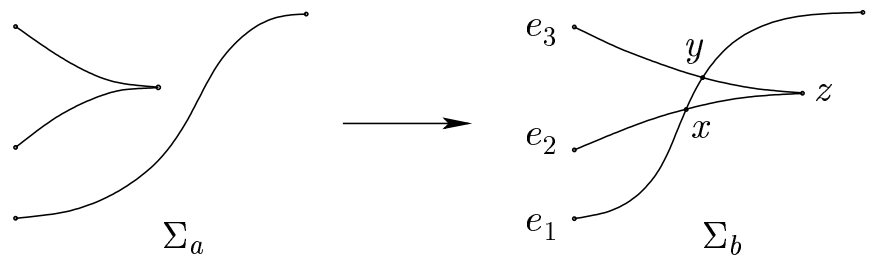

Рис. 7

ДокАЗАТЕльство. Предположим, что точка $y$ переключающая. Тогда в точке $x$ нарушено условие (PI4), так как ее окрестность содержит точки обоих Р-сечений, сходящихся в клюве $z$. Значит, точка $y$ непереключающая. Пусть точка $x$ переключаюшая, а $y$ непереключаюшая. Тогда точка $x$ отрицательная, так как в этом случае $\mathrm{P}\left(e_{1}\right)=e_{3}$ и $\mathrm{P}\left(e_{2}\right)$ не лежит на отрезке $\left[e_{1}, e_{3}\right]$.

Поскольку $\chi\left(\mathrm{P}_{b}\right)=\chi\left(\mathrm{P}_{a}\right)=1 / 2$ и у псевдоинволюций $\mathrm{P}_{a}, \mathrm{P}_{b}$ нет переключающих точек, семейство псевдоинволюций с требуемьми свойствами единственно по лемме 3.2 .

3.7. Перестройка $\mathrm{II}^{-1}$. Утверждение теоремы для случая перестройки $\mathrm{II}^{-1}$ таким же образом следует из леммы 3.3 .

3.8. Перестройка III. Разобьем случай перестройки III (см. рис. 8) на четыре подслучая $\mathrm{III}_{i}$, где $i$ - число переключаюших точек псевдоинволюции $\mathrm{P}_{a}$.
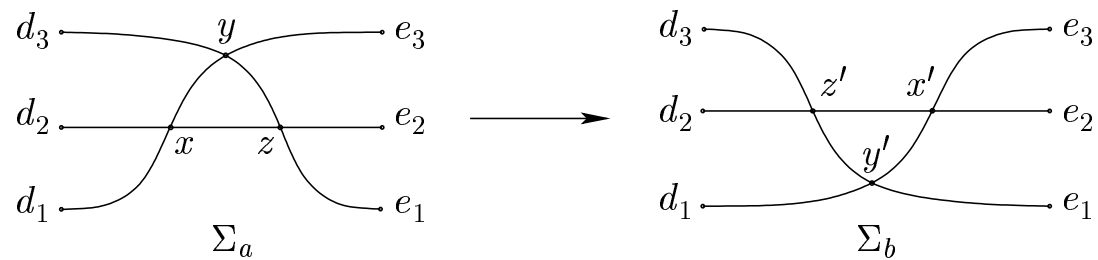

Рис. 8

Определим взаимно однозначное отображение $f_{a}:\left\{d_{1}, d_{2}, d_{3}\right\} \rightarrow\left\{e_{1}, e_{2}, e_{3}\right\}$ такое, что для каждого $i \in\{1,2,3\}$ найдется $\mathrm{P}_{a}$-сечение $\Gamma_{i}$, соединяющее $d_{i}$ с $f_{a}\left(d_{i}\right)$. Аналогичньм образом всякое разбиение $D$ фронта $\Sigma_{b}$ определяет отображение $f_{D}:\left\{d_{1}, d_{2}, d_{3}\right\} \rightarrow\left\{e_{1}, e_{2}, e_{3}\right\}$.

Лемма 3.4. Отображение, сопоставляющее псевдоинволюиии $\mathrm{P}_{b}$ фронта $\Sigma_{b}$ разбиение $D_{\mathrm{P}_{b}}$ фронта $\Sigma_{b}$, задает взаимно однозначное соответствие мехду множеством псевдоинволюиий фронта $\Sigma_{b}$, совпадающих с $\mathrm{P}_{a}$ на $\partial \Sigma_{a}=\partial \Sigma_{b}, u$ множсеством таких разбиений $D$ фронта $\Sigma_{b}$, что $f_{D}=f_{a}$.

Доказательство леммы 3.4 использует следуюшее утверждение.

ЛЕмма 3.5. Положительная псевдоинволющия $\mathrm{P}_{a}$ не переставляет никакую пару из точек $d_{i}, e_{i}, i \in\{1,2,3\}$. 
ДокАЗАТЕльство. Предположим, что $\mathrm{P}_{a}\left(d_{1}\right)=d_{2}$. Тогда в точке $x$ нарушается условие (PI4). Если $\mathrm{P}_{a}\left(d_{2}\right)=d_{3}$, то точка $x$ непереключающая (иначе условие (PI4) нарушается в точке $y$ ). Тогда точка $y$ переключаюшая (иначе условие (PI4) нарушается в точке $z$ ). Точка $y$ - отрицательная переключающая точка, противоречие. Пусть $\mathrm{P}_{a}\left(d_{1}\right)=d_{3}$. Тогда точка $x$ переключающая (иначе условие (PI4) нарушается в точке $y$ ). Получаем противоречие, так как точка $x$ отрицательна. Точки $e_{i}$ не переставляются по тем же причинам.

ДокАЗАТЕЛЬСтво ЛЕммы 3.4. Докажем, что $f_{D_{\mathrm{P}_{b}}}\left(d_{i}\right)=f_{a}\left(d_{i}\right)$ для всякого $i \in$ $\{1,2,3\}$. Согласно лемме 3.5 , сушествует такое сечение Г фронта $\Sigma_{a}$, состояшее из неособых точек, что $\mathrm{P}_{a}(\Gamma)$ содержит $d_{i}$ и $f_{a}\left(d_{i}\right)$. Сечение $\Gamma^{\prime}$ фронта $\Sigma_{b}$ с теми же концами, что и сечение $\Gamma$, отображается псевдоинволюцией $\mathrm{P}_{b}$ в $\mathrm{P}_{b}$-сечение, соединяющее $d_{i}$ и $f_{a}\left(d_{i}\right)$. Значит, отображение леммы 3.4 определено корректно.

Пусть $D$ - разбиение фронта $\Sigma_{b}$. Найдется ровно одна такая свободная инволюция $\theta: D \rightarrow D$, что при $\Gamma \in D, \Gamma^{\prime} \in D_{\mathrm{P}_{a}}$ левые конщы сечений $\theta(\Gamma), \theta_{\mathrm{P}_{a}}\left(\Gamma^{\prime}\right)$ совпадают тогда и только тогда, когда совпадают левые концы сечений $\Gamma, \Gamma^{\prime}$. Из леммы 3.5 следует, что для всякого $\Gamma \in D$ сечения $\theta(\Gamma)$ и $Г$ не пересекаются. Согласно утверждению 2.2 , существует ровно одна такая псевдоинволюция $\mathrm{P}_{b}$ фронта $\Sigma_{b}$, совпадающая с $\mathrm{P}_{a}$ над левым концом отрезка $I$, что $D=D_{P_{b}}$. Если $f_{D}=f_{a}$, то эти псевдоинволюции совпадают и над правым конщом отрезка $I$, и потому отображение леммы 3.4 обратимо.

3.9. Перестройка $\mathrm{III}_{0}$. Легко проверить, что существует ровно одно разбиение $D$ фронта $\Sigma_{b}$ с $f_{D}=f_{a}-$ то, для которого точки $z^{\prime}, y^{\prime}, x^{\prime}$ (см. рис. 8) непереключающие. Это разбиение задает (по лемме 3.4 ) псевдоинволюцию $\mathrm{P}_{b}$, при этом $\chi\left(\mathrm{P}_{b}\right)=\chi\left(\mathrm{P}_{a}\right)=0$. Поскольку у псевдоинволюции $\mathrm{P}_{b}$ нет переключающих точек на $\Sigma_{b}$, то она положительна (и масловская, если $\mathrm{P}_{a}$ масловская). Утверждение теоремы в этом случае получается применением леммы 3.2 .

3.10. Перестройка $\mathrm{III}_{1}$. Пусть $\mathrm{Sw}\left(\mathrm{P}_{a}\right)=\{x\}$. Разбиение $D$ фронта $\Sigma_{b}$, для которого $f_{D}=f_{a}$, единственно, $x^{\prime}$ - его единственная переключаюшая точка (из того, что $f_{D}\left(d_{3}\right)=e_{1}$, вытекает, что точки $z^{\prime}$ и $y^{\prime}$ непереключаюшие). По лемме 3.4 существует и единственна псевдоинволющия $\mathrm{P}_{b}$, совпадающая с $\mathrm{P}_{a}$ на $\partial \Sigma_{b}=\partial \Sigma_{a}$, при этом $\mathrm{Sw}\left(\mathrm{P}_{b}\right)=\left\{x^{\prime}\right\}$ и $\chi\left(\mathrm{P}_{b}\right)=\chi\left(\mathrm{P}_{a}\right)=-1$. Положительность точки $x^{\prime}$ следует из положительности точки $x$ и леммы 3.5 (см. также лемму 3.6 ниже). Масловость точки $x^{\prime}$ следует из масловости точки $x$. Утверждение теоремы для случая $\operatorname{Sw}\left(\mathrm{P}_{a}\right)=\{x\}$ следует из леммы 3.2. Случай $\mathrm{Sw}\left(\mathrm{P}_{a}\right)=\{z\}$ аналогичен.

Пусть $\mathrm{Sw}\left(\mathrm{P}_{a}\right)=\{y\}$. Тогда сушествует ровно два разбиения $D$ фронта $\Sigma_{b}$, для которых $f_{D}=f_{a}$. В первом случае все точки $z^{\prime}, y^{\prime}, x^{\prime}$ переключаюшие, но тогда $-3=\chi\left(\mathrm{P}_{b}\right) \neq \chi\left(\mathrm{P}_{a}\right)=-1$. Во втором случае $\mathrm{Sw}(D)=\left\{y^{\prime}\right\}$ и доказательство такое же, как для $\mathrm{Sw}\left(\mathrm{P}_{a}\right)=\{x\}$.

3.11. Леммы о знаках. Сформулируем и докажем некоторые вспомогательные утверждения, которые потребуются в дальнейшем. Пусть Р - псевдоинволюция $\sigma$-общего фронта $\Sigma \subset J^{0}(M), d_{1}$ и $d_{2}$ - такие различные неособые точки фронта, что $\pi\left(d_{1}\right)=\pi\left(d_{2}\right)$ и $\mathrm{P}\left(d_{1}\right) \neq d_{2}$. Определим знак $\varepsilon_{\mathrm{P}}\left(d_{1}, d_{2}\right)$ следующим образом: $\varepsilon_{\mathrm{P}}\left(d_{1}, d_{2}\right)=-1$, если пересечение отрезков $\left[d_{1}, \mathrm{P}\left(d_{1}\right)\right],\left[d_{2}, \mathrm{P}\left(d_{2}\right)\right]$ непусто и не совпадает ни с одним из этих отрезков; иначе полагаем $\varepsilon_{\mathrm{P}}\left(d_{1}, d_{2}\right)=+1$. Пусть $x \in \mathrm{Sw}(\mathrm{P})$. 
Положим $\varepsilon_{\mathrm{P}}(x)=+1$, если $x$ - положительная переключающая точка псевдоинволюции $\mathrm{P}$, и $\varepsilon_{\mathrm{P}}(x)=-1$ в противном случае (такую переключающую точку назовем отрицательной). Заметим, что знак переключающей точки - это знак пары близких к ней неособых точек. Следующее утверждение очевидно.

Лемма 3.6. Пусть $\mathrm{P}$ - псевдоинволющия $\sigma$-общего фронта $\Sigma \subset J^{0}(I), \Gamma_{1}, \Gamma_{2}$ - различные $\mathrm{P}$-сечения фронта $\Sigma$, не переставляемые инволюиией $\theta_{\mathrm{P}}$. Пусть $d_{1}, e_{1} \in \Gamma_{1}, d_{2}, e_{2} \in \Gamma_{2}-$ неособие точки фронта $\Sigma, \pi\left(d_{1}\right)=\pi\left(d_{2}\right), \pi\left(e_{1}\right)=\pi\left(e_{2}\right)$, точка $x$ - единственная точка самопересечения фронта $\Sigma$, проекиия которой на I лежит между $\pi\left(d_{1}\right)$ и $\pi\left(e_{1}\right)$. Тогда $\varepsilon_{\mathrm{P}}\left(d_{1}, d_{2}\right)=-\varepsilon_{\mathrm{P}}\left(e_{1}, e_{2}\right)$ тогда и только тогда, когда выполнены следующие два условия: (1) точка х непереключающая для $\mathrm{P} ;(2)$ в точке $x$ пересекаются два из сечений $\Gamma_{1}, \Gamma_{2}, \theta_{\mathrm{P}}\left(\Gamma_{1}\right), \theta_{\mathrm{P}}\left(\Gamma_{2}\right)$.

Лемма 3.7. Пусть $\mathrm{P}$ - псевдоинволюиия фронта $\Sigma \subset J^{0}(M), q \in M$, множество $\Sigma^{q}=\pi^{-1}(q) \cap \Sigma=\left\{h_{1}, \ldots, h_{2 N}\right\}$ состоит из неособых точек фронта $\Sigma$, занумерованных в порядке возрастания координаты и. Eсли $\varepsilon_{\mathrm{P}}\left(h_{k-1}, h_{k}\right)=$ $\varepsilon_{\mathrm{P}}\left(h_{k}, h_{k+1}\right)=+1$, mo $\varepsilon_{\mathrm{P}}\left(h_{k-1}, h_{k+1}\right)=+1$.

ДокАЗАТЕльство. Введем на $\Sigma^{q}$ отношение порядка, полагая $h_{k+2} \prec \cdots \prec h_{2 N}$ $\prec h_{1} \prec \cdots \prec h_{k+1}$. Из условия $\varepsilon_{\mathrm{P}}\left(h_{k-1}, h_{k}\right)=+1$ вытекает, что $\mathrm{P}\left(h_{k-1}\right) \neq h_{k+1}$. Поэтому при $k-1 \leqslant i<j \leqslant k+1$ равенство $\varepsilon_{\mathrm{P}}\left(h_{i}, h_{j}\right)=+1$ имеет место, если и только если $\mathrm{P}\left(h_{j}\right) \prec \mathrm{P}\left(h_{i}\right)$. Из $\mathrm{P}\left(h_{k}\right) \prec \mathrm{P}\left(h_{k-1}\right)$ и $\mathrm{P}\left(h_{k+1}\right) \prec \mathrm{P}\left(h_{k}\right)$ следует $\mathrm{P}\left(h_{k+1}\right) \prec \mathrm{P}\left(h_{k-1}\right)$.

3.12. Перестройка $\mathrm{III}_{2}$. Пусть $\mathrm{Sw}\left(\mathrm{P}_{a}\right)=\{x, y\}$. Единственное разбиение $D$ фронта $\Sigma_{b}$ с $f_{D}=f_{a}$ определяется условием $\operatorname{Sw}(D)=\left\{z^{\prime}, x^{\prime}\right\}$. По лемме 3.4 сушествует и единственна псевдоинволюция $\mathrm{P}_{b}$, совпадающая с $\mathrm{P}_{a}$ на $\partial \Sigma_{b}=\partial \Sigma_{a}$, при этом $\mathrm{Sw}\left(\mathrm{P}_{b}\right)=\left\{z^{\prime}, x^{\prime}\right\}$ и $\chi\left(\mathrm{P}_{b}\right)=\chi\left(\mathrm{P}_{a}\right)=-2$.

Докажем, что переключающие точки $z^{\prime}, x^{\prime}$ псевдоинволюции $\mathrm{P}_{b}$, соответствующей разбиению $D$, положительны. Знак $\varepsilon_{\mathrm{P}_{b}}\left(z^{\prime}\right)$ равен $\varepsilon_{\mathrm{P}_{b}}\left(d_{2}, d_{3}\right)=\varepsilon_{\mathrm{P}_{a}}\left(d_{2}, d_{3}\right)$. По лемме $3.6 \varepsilon_{\mathrm{P}_{a}}\left(d_{2}, d_{3}\right)=\varepsilon_{\mathrm{P}_{a}}(y)=+1$. Следовательно, $\varepsilon_{\mathrm{P}_{b}}\left(z^{\prime}\right)=\varepsilon_{\mathrm{P}_{a}}(y)=+1$. Применяя дважды лемму 3.6 , получаем $\varepsilon_{\mathrm{P}_{b}}\left(x^{\prime}\right)=\varepsilon_{\mathrm{P}_{b}}\left(d_{1}, d_{3}\right)=\varepsilon_{\mathrm{P}_{a}}\left(d_{1}, d_{3}\right)$. Знак $\varepsilon_{\mathrm{P}_{a}}\left(d_{1}, d_{2}\right)$ равен знаку $\varepsilon_{\mathrm{P}_{a}}(x)=+1$. По лемме $3.7 \varepsilon_{\mathrm{P}_{b}}\left(d_{1}, d_{3}\right)=+1$. Значит, точка $x^{\prime}$ положительна. Если $\mathrm{P}_{a}$ масловская, то масловский потенциал $\mu_{a}$ принимает одинаковые значения на прообразах всех ветвей, изображенных на рис. 8 , поскольку точки $x, y$ масловские. Поэтому псевдоинволюция $\mathrm{P}_{b}$ масловская. Утверждение теоремы для случая $\mathrm{Sw}\left(\mathrm{P}_{a}\right)=\{x, y\}$ доказано. Случай $\mathrm{Sw}\left(\mathrm{P}_{a}\right)=\{y, z\}$ рассматривается так же.

Рассмотрим случай $\mathrm{Sw}\left(\mathrm{P}_{a}\right)=\{x, z\}$. Существуют ровно два разбиения $D_{1}$ и $D_{2}$ фронта $\Sigma_{b}$ такие, что $f_{D_{1}}=f_{D_{2}}=f_{a}$, при этом $\operatorname{Sw}\left(D_{1}\right)=\left\{z^{\prime}, y^{\prime}\right\}, \operatorname{Sw}\left(D_{2}\right)=\left\{y^{\prime}, x^{\prime}\right\}$. Докажем, что ровно одна из двух псевдоинволюций $\mathrm{P}_{\langle i\rangle}$, соответствуюших разбиениям $D_{i}$, положительна. Так как $\varepsilon_{\mathrm{P}_{a}}(x)=+1$, то $\varepsilon_{\mathrm{P}_{a}}\left(d_{1}, d_{2}\right)=+1$. По лемме 3.6 из $\varepsilon_{\mathrm{P}_{a}}(z)=+1$ следует $\varepsilon_{\mathrm{P}_{a}}\left(d_{1}, d_{3}\right)=+1$. Так как $\varepsilon_{\mathrm{P}_{a}}\left(d_{1}, d_{2}\right)=+1$, то по лемме 3.6 $\varepsilon_{\mathrm{P}_{\langle 1\rangle}}\left(y^{\prime}\right)=+1$. Так как $\varepsilon_{\mathrm{P}_{a}}\left(d_{1}, d_{3}\right)=+1$, то по лемме $3.6 \varepsilon_{\mathrm{P}_{\langle 2\rangle}}\left(y^{\prime}\right)=+1$. Кроме того, $\varepsilon_{\mathrm{P}_{\langle 1\rangle}}\left(z^{\prime}\right)=\varepsilon_{\mathrm{P}_{a}}\left(d_{2}, d_{3}\right)$ и по лемме $3.6 \varepsilon_{\mathrm{P}_{\langle 2\rangle}}\left(x^{\prime}\right)=-\varepsilon_{\mathrm{P}_{a}}\left(d_{2}, d_{3}\right)$. Значит, ровно одна из псевдоинволюций $\mathrm{P}_{\langle 1\rangle}, \mathrm{P}_{\langle 2\rangle}$ является положительной: $\mathrm{P}_{\langle 1\rangle}$ при $\varepsilon_{\mathrm{P}_{a}}\left(d_{2}, d_{3}\right)=+1$, $\mathrm{P}_{\langle 2\rangle}$ при $\varepsilon_{\mathrm{P}_{a}}\left(d_{2}, d_{3}\right)=-1$. Вьполнено равенство $\chi\left(\mathrm{P}_{\langle 1\rangle}\right)=\chi\left(\mathrm{P}_{\langle 2\rangle}\right)=\chi\left(\mathrm{P}_{a}\right)=-2$. Доказательство масловости такое же, как для случая $\mathrm{Sw}\left(\mathrm{P}_{a}\right)=\{x, y\}$. 
3.13. Перестройка $\mathrm{III}_{3}$. В этом случае $\mathrm{Sw}\left(\mathrm{P}_{a}\right)=\{x, y, z\}$. Существуют ровно два разбиения $D$ фронта $\Sigma_{b}$ с $f_{D}=f_{a}$. В первом случае $\operatorname{Sw}\left(\mathrm{P}_{b}\right)=\left\{y^{\prime}\right\}$ и $\chi\left(\mathrm{P}_{b}\right) \neq$ $\chi\left(\mathrm{P}_{a}\right)$. Во втором случае $\mathrm{Sw}\left(\mathrm{P}_{b}\right)=\left\{z^{\prime}, y^{\prime}, x^{\prime}\right\}$. Применяя лемму 3.6 , легко убедиться, что положительность переключаюших точек $z^{\prime}, y^{\prime}, x^{\prime}$ следует из положительности переключаюших точек $x, y, z$. Окончание доказательства такое же, как для случая $\mathrm{III}_{2}$.

3.14. Перестройки $\mathrm{XC}, \mathrm{CC}_{ \pm}, \mathrm{XC}^{-1}, \mathrm{CC}_{-}^{-1}$. Утверждение теоремы для этих перестроек очевидно.

3.15. Перестройка ХХ. Разобьем случай этой перестройки (см. рис. 9) на три подслучая $\mathrm{XX}_{i}$, где $i$ - число переключающих точек псевдоинволющии $\mathrm{P}_{a}$. Продолжая псевдоинволюцию по непрерьвности, мы получаем следуюшее утверждение.
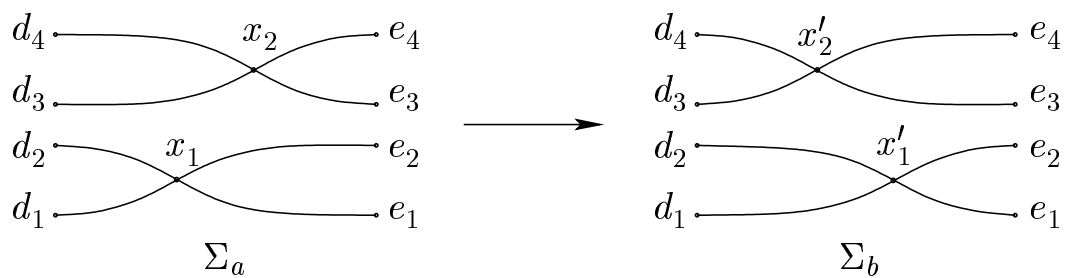

Рис. 9

ЛЕмма 3.8. В случае перестройки XX для всякой псевдоинволюиии $\mathrm{P}_{a}$ найдется такое непрерывное семейство $\left\{\mathrm{P}_{t \in[a, b]}^{\mathrm{S}}\right\}$ псевдоинволюиий с переключающими точками, гладко зависяшими от параметра $t$, что $\mathrm{P}_{a}^{\mathrm{S}}=\mathrm{P}_{a}$. Это семейство масловское, если $\mathrm{P}_{a}$ масловская.

3.16. Перестройка $\mathrm{XX}_{0}$. Из условия $\chi\left(\mathrm{P}_{b}\right)=\chi\left(\mathrm{P}_{a}\right)$ вытекает, что псевдоинволюция $\mathrm{P}_{b}$ не имеет переключающих точек. Существование требуемого семейства псевдоинволюций вытекает из леммы 3.8, единственность - из утверждения 2.3 и леммы 3.2.

3.17. Перестройка $\mathrm{XX}_{1}$. Обозначим через $\Gamma_{i} \mathrm{P}_{a}$-сечение фронта $\Sigma_{a}$, содержащее точку $d_{i}, i \in\{1,2,3,4\}$. Заметим, что $\theta\left(\Gamma_{1}\right) \neq \Gamma_{2}$, где $\theta=\theta_{\mathrm{P}_{a}}$.

Пусть $\operatorname{Sw}\left(\mathrm{P}_{a}\right)=\left\{x_{1}\right\}$. Рассмотрим случай $\left\{\theta\left(\Gamma_{1}\right), \theta\left(\Gamma_{2}\right)\right\} \neq\left\{\Gamma_{3}, \Gamma_{4}\right\}$. Пусть $\mathrm{P}^{\prime}-$ псевдоинволюция фронта $\Sigma_{b}$, которая совпадает с $\mathrm{P}_{a}$ на $\partial \Sigma_{b}=\partial \Sigma_{a}$. Докажем, что $\mathrm{Sw}\left(\mathrm{P}^{\prime}\right)=\left\{x_{1}^{\prime}\right\}$. Пусть $x_{1}^{\prime} \notin \mathrm{Sw}\left(\mathrm{P}^{\prime}\right)$. Найдется такое $i \in\{1,2\}$, что $\theta\left(\Gamma_{i}\right)=\Gamma_{5} \notin$ $\left\{\Gamma_{3}, \Gamma_{4}\right\}$. Тогда $\mathrm{P}_{a}\left(d_{i}\right)=d_{5}, \mathrm{P}_{a}\left(e_{i}\right)=e_{5}$, где $d_{5}, e_{5}-$ конщы $\mathrm{P}_{a}$-сечения $\Gamma_{5}$. Рассмотрим $\mathrm{P}^{\prime}$-сечения $\Gamma_{5}^{\prime}$ с конщами $d_{5}, e_{5}$ и $\Gamma_{i}^{\prime}$ с конщами $d_{i}, e_{3-i}$. Из $\mathrm{P}_{a}\left(d_{5}\right)=d_{i} \in \Gamma_{i}^{\prime}$ следует, что $\theta_{\mathrm{P}^{\prime}}\left(\Gamma_{5}^{\prime}\right)=\Gamma_{i}^{\prime}$, но $\mathrm{P}_{a}\left(e_{5}\right)=e_{i} \notin \Gamma_{i}^{\prime}$, противоречие. Пусть $\mathrm{Sw}\left(\mathrm{P}^{\prime}\right)=\left\{x_{1}^{\prime}, x_{2}^{\prime}\right\}$. Найдется такое $i \in\{3,4\}$, что $\theta\left(\Gamma_{i}\right)=\Gamma_{5} \notin\left\{\Gamma_{1}, \Gamma_{2}\right\}$. Рассмотрим $\mathrm{P}^{\prime}$-сечения $\Gamma_{5}^{\prime} \mathrm{c}$ концами $d_{5}, e_{5}$ и $\Gamma_{i}^{\prime}$ с конщами $d_{i}, e_{i}$. Из $\mathrm{P}_{a}\left(d_{5}\right)=d_{i} \in \Gamma_{i}^{\prime}$ следует, что $\theta_{\mathrm{P}^{\prime}}\left(\Gamma_{5}^{\prime}\right)=\Gamma_{i}^{\prime}$, но $\mathrm{P}_{a}\left(e_{5}\right)=e_{7-i} \notin \Gamma_{i}^{\prime}$, противоречие.

Теперь из леммы 3.8 вытекает, что в случае $\left\{\theta\left(\Gamma_{1}\right), \theta\left(\Gamma_{2}\right)\right\} \neq\left\{\Gamma_{3}, \Gamma_{4}\right\}$ существует и единственна псевдоинволюция $\mathrm{P}_{b}$, совпадающая с $\mathrm{P}_{a}$ на $\partial \Sigma_{b}=\partial \Sigma_{a}$, при этом $\mathrm{Sw}\left(\mathrm{P}_{b}\right)=\left\{x_{1}^{\prime}\right\}$. Эта псевдоинволюция положительна, поскольку, согласно лемме 3.6, $\varepsilon_{\mathrm{P}_{b}}\left(x_{1}^{\prime}\right)=\varepsilon_{\mathrm{P}_{b}}\left(e_{1}, e_{2}\right)=\varepsilon_{\mathrm{P}_{a}}\left(x_{1}\right)=+1$. Утверждение теоремы для этого случая следует из леммы 3.8 и леммы 3.2 . 
Предположим, что $\left\{\theta\left(\Gamma_{1}\right), \theta\left(\Gamma_{2}\right)\right\}=\left\{\Gamma_{3}, \Gamma_{4}\right\}$. Тогда легко убедиться в том, что найдутся ровно две псевдоинволюции $\mathrm{P}, \mathrm{P}^{\prime}$ фронта $\Sigma_{b}$, которые совпадают с $\mathrm{P}_{a}$ на $\partial \Sigma_{a}=\partial \Sigma_{b}$, при этом $\mathrm{Sw}(\mathrm{P})=\left\{x_{1}^{\prime}\right\}, \operatorname{Sw}\left(\mathrm{P}^{\prime}\right)=\left\{x_{2}^{\prime}\right\}$. Покажем, что $\mathrm{P}^{\prime}$ положительна, а $\mathrm{P}$ нет. Из положительности точки $x_{1}$ для псевдоинволюции $\mathrm{P}_{a}$ вытекает, что $\mathrm{P}\left(d_{1}\right)=\mathrm{P}^{\prime}\left(d_{1}\right)=\mathrm{P}_{a}\left(d_{1}\right)=d_{4}, \mathrm{P}\left(d_{2}\right)=\mathrm{P}^{\prime}\left(d_{2}\right)=\mathrm{P}_{a}\left(d_{2}\right)=d_{3}, \mathrm{P}\left(e_{1}\right)=e_{3}, \mathrm{P}\left(e_{2}\right)=e_{4}$. Значит, $\varepsilon_{\mathrm{P}}\left(x_{1}^{\prime}\right)=\varepsilon_{\mathrm{P}}\left(e_{1}, e_{2}\right)=-1, \varepsilon_{\mathrm{P}^{\prime}}\left(x_{2}^{\prime}\right)=\varepsilon_{\mathrm{P}}\left(d_{3}, d_{4}\right)+1$.

Если $\mathrm{P}_{a}$ масловская относительно $\mu_{a}$, то $\mu_{a}\left(d_{4}\right)=\mu_{a}\left(d_{1}\right)+1, \mu_{a}\left(d_{3}\right)=\mu_{a}\left(d_{2}\right)+1$, $\mu_{a}\left(d_{1}\right)=\mu_{a}\left(d_{2}\right)$. Следовательно, $\mu_{a}\left(d_{3}\right)=\mu_{a}\left(d_{4}\right)$, поэтому $\mu_{b}\left(d_{3}\right)=\mu_{b}\left(d_{4}\right)$ и псевдоинволюция $\mathrm{P}^{\prime}$ масловская. Применение леммы 3.2 завершает доказательство утверждения теоремы для перестройки $\mathrm{XX}_{1}$ (случай $\mathrm{Sw}\left(\mathrm{P}_{a}\right)=\left\{x_{2}\right\}$ сводится к случаю $\mathrm{Sw}\left(\mathrm{P}_{a}\right)=\left\{x_{1}\right\}$ симметрией $)$.

3.18. Перестройка $\mathrm{XX}_{2}$. Из условия $\chi\left(\mathrm{P}_{b}\right)=\chi\left(\mathrm{P}_{a}\right)$ вытекает, что обе точки самопересечения фронта $\Sigma_{b}$ должны быть переключаюшими. Непрерывное семейство $\left\{\mathrm{P}_{t}\right\}$ псевдоинволющий с двумя переключаюшими точками существует по лемме 3.8 , единственно по утверждению 2.3 и лемме 3.2 . Остается проверить, что $\mathrm{P}_{b}$ положительна. Из того, что $\varepsilon_{\mathrm{P}_{a}}\left(x_{1}\right)=\varepsilon_{\mathrm{P}_{a}}\left(x_{2}\right)=+1$, применением леммы 3.6 получаем $\varepsilon_{\mathrm{P}_{a}}\left(e_{1}, e_{2}\right)=\varepsilon_{\mathrm{P}_{a}}\left(d_{3}, d_{4}\right)=+1$. Псевдоинволющия $\mathrm{P}_{b}$ положительна, так как $\varepsilon_{\mathrm{P}_{b}}\left(x_{1}^{\prime}\right)=\varepsilon_{\mathrm{P}_{b}}\left(e_{3}, e_{4}\right)=\varepsilon_{\mathrm{P}_{a}}\left(e_{3}, e_{4}\right)=+1$ и $\varepsilon_{\mathrm{P}_{b}}\left(x_{2}^{\prime}\right)=\varepsilon_{\mathrm{P}_{b}}\left(d_{1}, d_{2}\right)=\varepsilon_{\mathrm{P}_{a}}\left(d_{1}, d_{2}\right)=+1$. Доказательство теоремы 2.5 закончено.

\section{§ 4. Неоднозначность непрерывного продолжения и монодромия псевдоинволюций}

4.1. Нехарактеристические продолжения. Оказьвается, если в условии теоремы 2.5 отбросить требование характеристичности семейства $\left\{\mathrm{P}_{t}\right\}$, то однозначность продолжения нарушается, при этом всевозможные продолжения псевдоинволюции $\mathrm{P}_{a}$ до непрерьвного положительного (не обязательно характеристического) семейства $\left\{\mathrm{P}_{t \in[a, b]}\right\}$ псевдоинволюций по-прежнему допускают явное описание. Таких продолжений не больше двух, если $\sigma$-обшее семейство $\left\{L_{t}\right\}$ пересекает дискриминант $\mathscr{D}$ ровно в одной точке. Их описание извлекается из доказательства теоремы 2.5; изложим его явно. Мы предполагаем, что семейство $\left\{L_{t}\right\}$ простое.

4.2. Однозначные продолжения. Если перестройка - это одна из перестроек I, II, $\mathrm{XC}$ и $\mathrm{CC}_{ \pm}$(или обратная к одной из них, см. п. 3.1), то такое непрерьвное положительное семейство $\left\{\mathrm{P}_{t}\right\}$ единственно. Это же верно, если перестройка - это перестройка типа $\mathrm{III}_{0}, \mathrm{III}_{2}$ (см. пп. 3.9 и 3.12 ) или типа $\mathrm{XX}_{1}$ (см. п. 3.17 ).

4.3. Неоднозначные продолжения. Если перестройка имеет тип $\mathrm{III}_{3}$, то всегда сушествуют ровно два непрерывных положительных семейства $\left\{\mathrm{P}_{t}\right\}$ : одно из них характеристично, а у другого число переключающих точек уменьшается на 2 (в обозначениях рис. 8 из переключаюших точек $x, y, z$ получается переключающая точка $\left.y^{\prime}\right)$.

Пусть перестройка имеет тип $\mathrm{III}_{1}$. Если переключаюшая точка для $\mathrm{P}_{a}$ это $x$ или $z$, то продолжение единственно. Пусть переключающая точка - это $y$. Допустим, что сушествует псевдоинволюция $\mathrm{P}_{a}^{\prime}$, совпадающая с $\mathrm{P}_{a}$ на $\partial \Sigma_{a}$, для которой $x, y$, $z$ - переключающие точки. Тогда псевдоинволюция $\mathrm{P}_{a}$ допускает два продолжения - 
одно указано в теореме 2.5 , второе получается из семейства $\left\{\mathrm{P}_{t}^{\prime}\right\}$, продолжающего $\mathrm{P}_{a}^{\prime}$, по теореме 2.5. Если такой псевдоинволюции $\mathrm{P}_{a}^{\prime}$ не существует, то продолжение единственно.

Пусть перестройка имеет тип $\mathrm{XX}_{0}$ или $\mathrm{XX}_{2}$. Продолжение псевдоинволющии $\mathrm{P}_{a}$ неоднозначно, если и только если $\mathrm{P}_{a}\left(d_{1}\right)=d_{4}, \mathrm{P}_{a}\left(d_{2}\right)=d_{3}$ (см. рис. 9). Тогда продолжений ровно два: для одного из них точки $x_{1}^{\prime}, x_{2}^{\prime}$ переключающие, для другого обе эти точки непереключаюшие.

4.4. Характеристические продолжения и монодромия. Пусть $\left\{L_{t \in[0,1]}\right\}-$ $\sigma$-общий путь ( $\sigma$-общее семейство) в пространстве лежандровых зацеплений в $J^{1}(M)$. Продеформируем путь $\left\{L_{t}\right\}$ в другой $\sigma$-обший путь $\left\{L_{t}^{\prime}\right\}$ в классе путей в $\mathscr{L}$ с фиксированными конщами. Согласно утверждению 2.6, эти пути определяют взаимно однозначные отображения $\Phi, \Phi^{\prime}$ из множества положительных псевдоинволюций фронта $\sigma\left(L_{0}\right)$ в множество положительных псевдоинволюций фронта $\sigma\left(L_{1}\right)$. Оказьвается, что эти отображения не всегда совпадают.

Легко проверить, что если в процессе дефоормации встречаются лишь $\sigma$-общие пути, то $\Phi=\Phi^{\prime}$. Более того, можно показать (пользуясь обратимостью продолжения псевдоинволюции вдоль пути), что равенство $\Phi=\Phi^{\prime}$ справедливо, если всякое лежандрово зацепление, участвующее в деформащии, либо является $\sigma$-общим, либо является неособой точкой дискриминанта $\mathscr{D}$. Таким образом, неоднозначность продолжения определяется обходами вокруг стратов коразмерности 2 (в $\mathscr{L}$ ) дискриминанта $\mathscr{D}$. Доказательство теоремы 2.5 доставляет нам явное описание продолжения псевдоинволюции вдоль пути. Пользуясь им, можно описать взаимно однозначные отображения множества положительных псевдоинволюций (преобразования монодромии) при обходе вокруг различных стратов дискримината коразмерности 2. Полное описание утомительно, мы сформулируем лишь некоторые из результатов.

Лишь для двух стратов преобразование монодромии может быть отличным от тождественного. Опишем эти страты и некоторые свойства монодромии вокруг них. Страт $\mathscr{D}_{2,3}$ состоит из таких лежандровых зацеплений $L$, что фронт $\sigma(L)$ имеет тройную точку $x$, двойную точку $x^{\prime}$, вьполняется условие $\pi(x)=\pi\left(x^{\prime}\right)$, а в остальном фронт является $\sigma$-обшим. Рассмотрим малую $\sigma$-обшую петлю $\left\{L_{t \in[0,1]}\right\}$, один раз обходящую страт $\mathscr{D}_{2,3}$ вблизи $L$. Можно показать, что квадрат отображения монодромии вокруг этой петли тождественен. Положительные псевдоинволющии, не переходщие в себя при отображении монодромии вдоль этой петли, содержатся в множестве таких псевдоинволюций $\mathrm{P}$ фронта $\sigma\left(L_{0}\right)$, что ровно две точки множества $\mathrm{Sw}(\mathrm{P})$ близки к точкам $x, x^{\prime}$.

Страт $\mathscr{D}_{4}$ состоит из таких лежандровых зацеплений $L$, что фронт $\sigma(L)$ имеет четверную точку, а в остальном является $\sigma$-обшим. Рассмотрим малую $\sigma$-общую петлю $\left\{L_{t \in[0,1]}\right\}$, один раз обходяшую страт $\mathscr{D}_{4}$ вблизи $L$. Обозначим через $\Phi$ отображение монодромии множества положительных псевдоинволющий фронта $\sigma\left(L_{0}\right)$, через $A_{i}$ множество таких положительных псевдоинволюций $\mathrm{P}$ фронта $\sigma\left(L_{0}\right)$, что ровно $i$ точек множества $\mathrm{Sw}(\mathrm{P})$ близки к четверной точке фронта $\sigma(L)$. Отображение $\Phi$ сохраняет каждое из множеств $A_{i}$. Можно показать, что ограничение $\Phi$ на $A_{i}$ тождественно, если $i \notin\{3,4\}$, третья степень ограничения $\Phi$ на $A_{4}$ и квадрат ограничения $\Phi$ на $A_{3}$ тождественны.

Проиллюстрируем эти явления примерами. 
4.5. Первый пример. Рассмотрим лежандров узел $L \in \mathscr{D}_{2,3}$, фронт которого изображен на рис. 10 a. Пусть петля $\left\{L_{t \in[0,1]}\right\}$ один раз обходит вокруг страта $\mathscr{D}_{2,3}$ в окрестности узла $L$, фронт $\sigma\left(L_{0}\right)$ таков, как показано на рис. $10 \mathrm{~b}$. Мы предполагаем для простоты, что семейство $\left\{L_{t}\right\}$ пересекает страт $\mathscr{D}_{3}$ (состояший из фронтов с одной тройной точкой) и страт $\mathscr{D}_{2,2}$ (состояший из фронтов с двумя двойными точками, у которых совпадают $q$-координаты) минимальное число раз -2 и 6 соответственно. Фронт $\sigma\left(L_{0}\right)$ допускает ровно три положительные псевдоинволюции $\mathrm{P}^{0}, \mathrm{P}^{1}, \mathrm{P}^{2}$. Они задаются условиями $\mathrm{Sw}\left(\mathrm{P}^{0}\right)=\left\{x_{2}, x_{4}\right\}, \operatorname{Sw}\left(\mathrm{P}^{1}\right)=\left\{x_{3}, x_{4}\right\}$ и $\mathrm{Sw}\left(\mathrm{P}^{2}\right)=\left\{x_{1}, x_{2}, x_{3}, x_{4}\right\}$. Используя явное описание продолжения псевдоинволюций $\mathrm{P}_{0} \in\left\{\mathrm{P}^{0}, \mathrm{P}^{1}, \mathrm{P}^{2}\right\}$, данное в доказательстве теоремы 2.5 , можно показать, что обход вдоль петли $\left\{L_{t}\right\}$ переставляет псевдоинволюции $\mathrm{P}^{0}$ и $\mathrm{P}^{1}$.

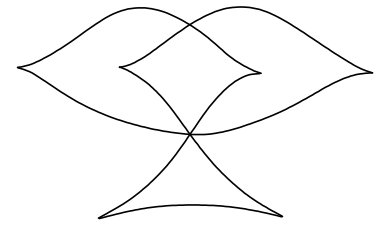

a

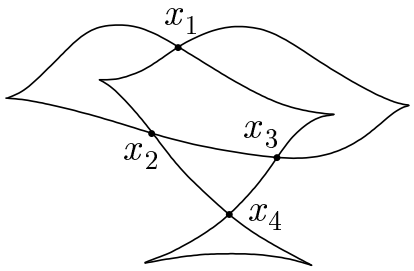

b

Рис. 10

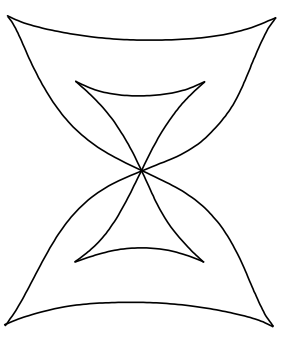

a

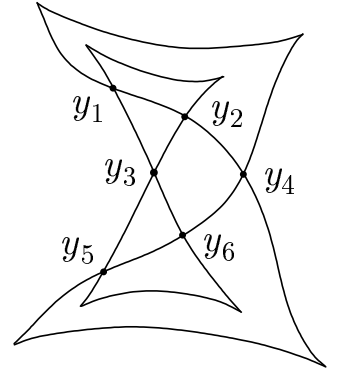

$\mathrm{b}$

Рис. 11

4.6. Второй пример. Рассмотрим лежандрово зацепление $L \in \mathscr{D}_{4}$, фронт которого изображен на рис. 11 а. Пусть путь $\left\{L_{t}\right\}$ обходит один раз вокруг страта $\mathscr{D}_{4}$ в окрестности зацепления $L$, фронт $\sigma\left(L_{0}\right)$ изображен на рис. $11 \mathrm{~b}$. Мы предполагаем для простоты, что семейство $\left\{L_{t}\right\}$ пересекает страт $\mathscr{D}_{3}$ и страт $\mathscr{D}_{2,2}$ минимальное число раз -8 и 6 соответственно. Фронт $\sigma\left(L_{0}\right)$ допускает ровно пять положительных псевдоинволюций $\mathrm{P}^{0}, \mathrm{P}^{1}, \mathrm{P}^{2}, \mathrm{P}^{3}, \mathrm{P}^{4}$. Они задаются условиями $\operatorname{Sw}\left(\mathrm{P}^{0}\right)=$ $\left\{y_{3}, y_{4}\right\}, \mathrm{Sw}\left(\mathrm{P}^{1}\right)=\left\{y_{3}, y_{4}, y_{5}, y_{6}\right\}, \mathrm{Sw}\left(\mathrm{P}^{2}\right)=\left\{y_{1}, y_{2}, y_{3}, y_{4}\right\}, \mathrm{Sw}\left(\mathrm{P}^{3}\right)=\left\{y_{1}, y_{2}, y_{5}, y_{6}\right\}$, $\mathrm{Sw}\left(\mathrm{P}^{4}\right)=\left\{y_{1}, y_{2}, y_{3}, y_{4}, y_{5}, y_{6}\right\}$. Используя явное описание продолжения псевдоинволюций $\mathrm{P}_{0} \in\left\{\mathrm{P}^{1}, \mathrm{P}^{2}, \mathrm{P}^{3}\right\}$, можно показать, что эти три псевдоинволюции циклически 
переставляются при обходе вдоль петли $\left\{L_{t}\right\}$ (а псевдоинволюции $\mathrm{P}^{0}$ и $\mathrm{P}^{4}$ переходят в себя, например, потому, что в силу характеристичности продолжения отображение монодромии сохраняет число переключающих точек).

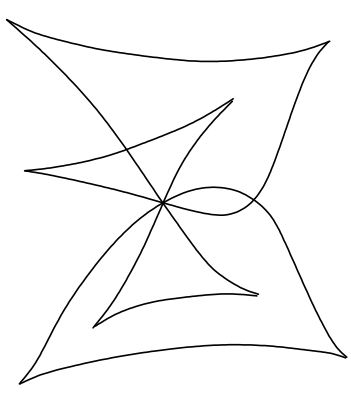

a

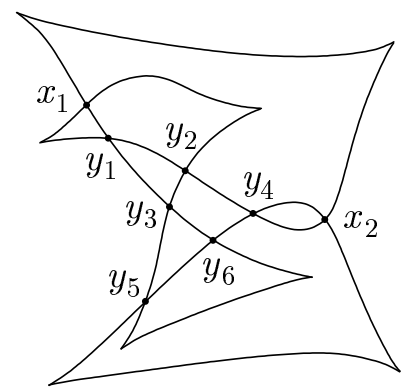

$\mathrm{b}$

Рис. 12

4.7. Третий пример. Рассмотрим лежандрово зацепление $L \in \mathscr{D}_{4}$, фронт которого изображен на рис. 12 а. Пусть путь $\left\{L_{t}\right\}$ обходит один вокруг страта $\mathscr{D}_{4}$ в окрестности зацепления $L$, фронт $\sigma\left(L_{0}\right)$ изображен на рис. $12 \mathrm{~b}$. $\Phi$ ронт $\sigma\left(L_{0}\right)$ допускает ровно пять положительных псевдоинволюций $\mathrm{P}^{0}, \mathrm{P}^{1}, \mathrm{P}^{2}, \mathrm{P}^{3}, \mathrm{P}^{4}$. Они задаются условиями $\mathrm{Sw}\left(\mathrm{P}^{0}\right)=\left\{y_{2}, y_{3}\right\}, \mathrm{Sw}\left(\mathrm{P}^{1}\right)=\left\{x_{2}, y_{2}, y_{5}, y_{6}\right\}, \mathrm{Sw}\left(\mathrm{P}^{2}\right)=\left\{x_{2}, y_{2}, y_{3}, y_{4}\right\}$, $\mathrm{Sw}\left(\mathrm{P}^{3}\right)=\left\{y_{2}, y_{3}, y_{5}, y_{6}\right\}, \mathrm{Sw}\left(\mathrm{P}^{4}\right)=\left\{x_{2}, y_{2}, y_{3}, y_{4}, y_{5}, y_{6}\right\}$. Используя явное описание продолжения псевдоинволющий $\mathrm{P}_{0} \in\left\{\mathrm{P}^{1}, \mathrm{P}^{2}\right\}$, можно показать, что эти две псевдоинволюции циклически переставляются при обходе вдоль петли $\left\{L_{t}\right\}$ (а псевдоинволюции $\mathrm{P}^{0}, \mathrm{P}^{3}$ и $\mathrm{P}^{4}$ переходят в себя).

Заметим, что зацепление $L$ принадлежит той же связной компоненте пространства лежандровых зацеплений, что и зацепление из предыдущего примера. Из этого несложно заключить, что всякая перестановка псевдоинволюций $\mathrm{P}^{1}, \mathrm{P}^{2}, \mathrm{P}^{3}$ фронта $\sigma\left(L_{0}\right)$ реализуется отображением монодромии вдоль некоторой $\sigma$-общей петли.

\section{§ 5. Продолжение псевдоинволюций на дискриминант}

5.1. Пусть $k$-целое число. Рассмотрим множество $\mathscr{L}_{k}^{\sigma}$ всех $\sigma$-обших лежандровых зацеплений в $J^{1}(M)$, фронты которых допускают ровно одну положительную псевдоинволюцию с эйлеровой характеристикой, равной $k$. Обозначим через $\mathscr{L}_{k}^{\prime}$ его замыкание в пространстве лежандровых зацеплений $\mathscr{L}$. Из утверждения 2.6 вытекает, что $\mathscr{L}_{k}^{\prime}$ есть объединение некоторых связных компонент пространства $\mathscr{L}$.

Рассмотрим отображение $\mathscr{P}_{0}$, сопоставляющее каждому лежандрову зацеплению $L \in \mathscr{L}_{k}^{\sigma}$ такую положительную псевдоинволюцию $\mathscr{P}_{0}(L)$ фронта $\sigma(L)$, что $\chi\left(\mathscr{P}_{0}(L)\right)=k$. Нашей целью является продолжение отображения $\mathscr{P}_{0}$ до непрерьвного отображения $\mathscr{P}$, определенного на $\mathscr{L}_{k}^{\prime}$ и сопоставляющего лежандрову зацеплению псевдоинволюцию его фронта. Непрерывность здесь понимается в следующем смысле. Рассмотрим множество $A_{\mathscr{P}}=\left\{(x, L) \mid x \in G_{\sigma(L)} \cup C_{\sigma(L)}\right\} \subset J^{0}(M) \times \mathscr{L}_{k}^{\prime}$ и отображение $\Phi_{\mathscr{P}}: A_{\mathscr{P}} \rightarrow J^{0}(M), \Phi_{\mathscr{P}}(x, L)=\mathscr{P}(L)(x)$. Снабдим множество $A_{\mathscr{P}}$ топологией, 
индуцированной из произведения естественной топологии на $J^{0}(M)$ и $C^{\infty}$-топологии на $\mathscr{L}_{k}^{\prime}$. Назовем отображение $\mathscr{P}$ непрерывнылм, если $\Phi \mathscr{P}$ непрерывно.

Теорема 5.1. Отображение $\mathscr{P}_{0}$ единственным образом продолжается до непрерывного отображения ЯР, сопоставляющего каждому лежандрову зачеплению $L \in \mathscr{L}_{k}^{\prime}$ псевдоинволюцию фронта $\sigma(L)$.

5.2. Доказательство теоремы 5.1. Если $x$ - клюв фронта $\Sigma=\sigma(L)$, то положим $\mathscr{P}(L)(x)=x$. Если $x$-неособая точка фронта $\Sigma$, то рассмотрим последовательность $\left(L_{i}\right)$ лежандровых зацеплений из $\mathscr{L}_{k}^{\sigma}$ и последовательность точек $x_{i} \in \sigma\left(L_{i}\right)$, где $L_{i} \rightarrow L, x_{i} \rightarrow x$. Положим $\mathscr{P}(L)(x)=\lim _{i \rightarrow \infty} \mathscr{P}_{0}\left(L_{i}\right)\left(x_{i}\right)$. Покажем, что это определение корректно и отображение $\mathscr{P}$ обладает требуемьми свойствами.

Пусть $\Gamma$ - множество, которое состоит из неособых точек фронта $\Sigma$ и является графиком гладкой функции, определенной на некотором отрезке $I \subset M$. Обозначим через $W_{\varepsilon}(\Gamma) \varepsilon$-окрестность в $J^{0}(I)$ множества $\Gamma$. Возьмем $\varepsilon>0$ достаточно малым, тогда окрестность $W_{\varepsilon}(\Gamma)$ не содержит точек фронта $\Sigma$, не принадлежащих $\Gamma$. Назовем лежандрово зацепление $L^{\prime} \quad \Gamma$-удобным, если пересечение его фронта с $W_{\varepsilon}(\Gamma)$ состоит из неособых точек фронта и является графиком некоторой гладкой функции на $I$. Пусть $L^{\prime} \in \mathscr{L}_{k}^{\sigma}-\sigma$-обшее Г-удобное зацепление, $\mathrm{P}^{\prime}=\mathscr{P}_{0}\left(L^{\prime}\right)$ - псевдоинволюция фронта $\sigma\left(L^{\prime}\right)$. Тогда множество $\mathrm{P}^{\prime}\left(\sigma\left(L^{\prime}\right) \cap W_{\varepsilon}(\Gamma)\right)$ является графиком непрерьвной функции на $I$. Обозначим эту функцию через $f_{L^{\prime}}$.

Лемма 5.2. Для любого $\delta>0$ найдется такая окрестность $U$ лежандрова зацепления $L$ в $\mathscr{L}_{k}^{\prime}$, состоящая из Г-удобных защеплений, что $\left\|f_{L_{1}}-f_{L_{2}}\right\|_{C^{0}}<\delta$ для любьх $L_{1}, L_{2} \in \mathscr{L}_{k}^{\sigma} \cap U$.

Докажем вначале следуюшее вспомогательное утверждение.

Лемма 5.3. Пусть $L_{0} \subset J^{1}(M)$-лежсандрово зачепление, $T^{L_{0}}$-множество, состоящее из всех таких точек $q_{0} \in M$, что прямая $\left\{q=q_{0}\right\}$ пересекает фронт $\sigma\left(L_{0}\right)$ лишь в его неособых точках. Тогда $T^{L_{0}}$ открыто и всюду плотно в $M$.

ДокАЗАТельство. Очевидно, что $T^{L_{0}}$ открыто в $M$. По лемме Сарда множество критических значений проекции $L_{0} \rightarrow M$ замкнуто и имеет меру нуль. Покажем, что на каждом отрезке $K \subset M$, состоящем из неособых значений этой проекции, найдется точка множества $T^{L_{0}}$. Действительно, множество $L_{0}^{K}=L_{0} \cap J^{1}(K)$ есть объединение конечного числа 1-графиков гладких функций $f_{1}, \ldots, f_{m}$ на $K$. Поскольку графики этих функций не касаются друг друга (в силу вложенности $L_{0}$ ), они попарно пересекаются лиш в конечном числе точек. Следовательно, почти все точки отрезка $K$ принадлежат множеству $T^{L_{0}}$. Поэтому множество $T^{L_{0}}$ всюду плотно в $M$. Очевидно, что $T^{L_{0}}$ открыто в $M$.

ДокАЗАТЕЛЬСТво ЛЕммы 5.2. Всякая достаточно малая окрестность лежандрова зацепления $L$ в $\mathscr{L}_{k}^{\prime}$ состоит из $Г$-удобных зацеплений. Покажем, что для любого числа $\delta_{0}>0$ и для любой точки $s \in T^{L_{0}} \cap I$ найдется такая окрестность $U_{s, \delta_{0}}$ зацепления $L$ в $\mathscr{L}_{k}^{\prime}$, что $\left|f_{L_{1}}(s)-f_{L_{2}}(s)\right|<\delta_{0}$ для любых $L_{1}, L_{2} \in \mathscr{L}_{k}^{\sigma} \cap U_{s, \delta_{0}}$. Действительно, выберем вначале связную окрестность $U_{s, \delta_{0}}$ такой, что точка $s$ принадлежит множеству $T^{L_{0}}$ для любого лежандрова зацепления $L_{0}$ из этой окрестности. Пусть $L_{0} \in \mathscr{L}_{k}^{\sigma} \cap U_{s, \delta_{0}}$. Обозначим через $x_{1}^{L_{0}}, x_{2}^{L_{0}}, \ldots, x_{2 n}^{L_{0}}$ точки множест- 
ва $\sigma\left(L_{0}\right) \cap\left\{q=q_{0}\right\}$, занумерованные в порядке возрастания координаты $u$. Псевдоинволюция $\mathscr{P}_{0}\left(L_{0}\right)$ определяет инволюцию $\theta$ множества $\{1, \ldots, 2 m\}$ при помощи формулы $\mathscr{P}_{0}\left(L_{0}\right)\left(x_{j}^{L_{0}}\right)=x_{\theta(j)}^{L_{0}}$. Построенные по разным лежандровым зацеплениям $L_{0} \in \mathscr{L}_{k}^{\sigma} \cap U_{s, \delta_{0}}$ инволюции $\theta$ совпадают. Это следует из теоремы 2.5 и того, что любые два такие зацепления могут быть соединены $\sigma$-общим семейством лежандровых зацеплений из $U_{s, \delta_{0}}$. Из совпадения инволюций вытекает, что найдется такое $j \in\{1, \ldots, 2 m\}$, что число $f_{L_{0}}(s)$ равно $u$-координате точки $x_{j}^{L_{0}}$ для любого $L_{0} \in \mathscr{L}_{k}^{\sigma} \cap U_{s, \delta_{0}}$. Поэтому, уменьшая окрестность $U_{s, \delta_{0}}$, мы можем добиться выполнения неравенства $\left|f_{L_{1}}(s)-f_{L_{2}}(s)\right|<\delta_{0}$.

Найдутся такое число $C>0$ и такая окрестность $U_{C}$ лежандрова зацепления $L$ в $\mathscr{L}_{k}^{\prime}$, что значение $p$-координаты не превосходит по модулю числа $C$. Тогда $\left|f_{L_{0}}\left(s_{1}\right)-f_{L_{0}}\left(s_{2}\right)\right| \leqslant C\left|s_{1}-s_{2}\right|$ для любых $L_{0} \in \mathscr{L}_{k}^{\sigma} \cap U_{C}, s_{1}, s_{2} \in I$. Найдутся такие положительные $\varepsilon_{0}, \delta_{0}$, что $\delta_{0}+2 C \varepsilon_{0}<\delta$. Для любой точки $s \in T^{L_{0}} \cap I$ неравенство $\left|f_{L_{1}}\left(s_{1}\right)-f_{L_{2}}\left(s_{1}\right)\right|<\delta$ справедливо для любой точки $s_{1}$ из $\varepsilon_{0}$-окрестности точки $s$ в $I$ и любых лежандровых зацеплений $L_{1}, L_{2} \in \mathscr{L}_{k}^{\sigma} \cap U_{s}, \delta_{0}$. По лемме 5.3 множество $T^{L_{0}} \cap I$ всюду плотно в $I$, поэтому в нем найдутся такие точки $\left\{s_{1}, \ldots, s_{l}\right\}$, что их $\varepsilon_{0}$-окрестности покрьвают отрезок $I$. Искомая окрестность $U$ есть пересечение окрестностей $U_{s_{i}, \delta_{0}}$.

Вернемся к доказательству теоремы 5.1. Покажем, что приведенное выше определение отображения $\mathscr{P}(L): G_{\sigma(L)} \cup C_{\sigma(L)} \rightarrow \sigma(L)$ корректно. Пусть $x$ - неособая точка фронта $\sigma(L),\left(L_{i}\right)$ - последовательность лежандровых зацеплений из $\mathscr{L}_{k}^{\sigma}$ и $x_{i} \in \sigma\left(L_{i}\right)$, $L_{i} \rightarrow L, x_{i} \rightarrow x$. Рассмотрим множество $\Gamma$, которое состоит из неособых точек фронта $\sigma(L)$, содержит точку $x$ и является графиком гладкой функции, определенной на некотором отрезке $I \subset M$. По лемме 5.2 последовательность функций $f_{L_{i}}: I \rightarrow \mathbb{R}$ сходится в пространстве $C^{0}$ к некоторой функции $f$. Поэтому последовательность $\mathscr{P}_{0}\left(L_{i}\right)\left(x_{i}\right)$ сходится к $(\pi(x), f(\pi(x)))$ (где $\pi$ - проекция $\left.J^{0}(M) \rightarrow M\right)$. Следовательно, отображение $\mathscr{P}$ определено корректно.

Отображение $\mathscr{P}(L)$ удовлетворяет всем требованиям, предъявляемьм к псевдоинволюциям. Это следует по соображениям непрерывности из того, что все отображения $\mathscr{P}_{0}\left(L^{\prime}\right)$ для $\sigma$-общих лежандровых зацеплений $L^{\prime}$, близких к $L$, являются псевдоинволюциями. Покажем, что отображение $\Phi \mathscr{P}$ непрерывно в точке $(x, L)$, где $x \in$ $G_{\sigma(L)}$. В силу леммы 5.2 достаточно показать, что точка $\mathscr{P}\left(L^{\prime}\right)\left(x^{\prime}\right)$ достаточно близка к точке $\mathscr{P}(L)(x)$, если зацепление $L^{\prime} \in \mathscr{L}_{k}^{\sigma}$ достаточно близко к $L, x^{\prime}$ - неособая точка фронта $\sigma\left(L^{\prime}\right)$, достаточно близкая к точке $x$. Последнее также доказывается применением леммы 5.2. Наконец, непрерьвность $\Phi \mathscr{P}$ в точке $(x, L)$, где $x$ - клюв, вытекает из того, что пересечение фронта всякого близкого к $L$ лежандрова зацепления $L^{\prime}$ с малой окрестностью $V$ точки $x$ в $J^{0}(M)$ получается из $V \cap \sigma(L)$ при помощи $C^{\infty}$-малого диффеоморфизма. Доказательство теоремы 5.1 закончено.

\section{§. Комбинаторика разбиений фронтов}

6.1. Разрешение. По разбиению $D=\left\{\gamma_{1}, \ldots, \gamma_{N}\right\} \sigma$-обшего фронта $\Sigma=\sigma(L) \subset$ $J^{0}(M)$ (где $M=S^{1}$, или $M=\mathbb{R}$, или $M=I$ ) построим топологическое многообразие $R(D)$ (с краем, если $M=I)$, назьваемое разрешением (D-разрешением) фронта $\Sigma$. Разрешение $R(D)$ получается из $\bigcup_{i=1}^{N} \Lambda_{i}$ (где $\gamma_{i}: \Lambda_{i} \rightarrow \Sigma$, отрезки и окружности $\Lambda_{i}$ попарно не пересекаются) склеиванием для каждого клюва $c$ фронта $\Sigma$ двух 
таких концов $y \in \Lambda_{j}, y^{\prime} \in \Lambda_{j^{\prime}}$, что $\gamma_{j}(y)=\gamma_{j^{\prime}}\left(y^{\prime}\right)=c$. Отображения $\gamma_{i}$ определяют естественную непрерывную проекцию $\psi_{D}: R(D) \rightarrow \Sigma$, при которой всякая точка самопересечения имеет ровно два прообраза, а остальные точки - по одному.

6.2. Древесные разбиения. Пусть $L \subset J^{1}\left(S^{1}\right)$ - $\sigma$-общее лежандрово зацепление, $D$ - разбиение его фронта $\Sigma \subset J^{0}\left(S^{1}\right)$. Обозначим через $\mathrm{Sw}_{L}(D) \subset L$ прообраз множества $\operatorname{Sw}(D)$ под действием отображения $\left.\sigma\right|_{L}$.

Сопоставим разбиению $D$ граф $K_{D}$ следуюшим образом. Вершины графа $K_{D}$ соответствуют связным компонентам пространства $R(D)$. По каждой точке $x \in \mathrm{Sw}(D)$ строится ребро графа $K_{D}$, которое соединяет (возможно, совпадаюшие) вершины, соответствующие компонентам, содержашим прообразы точки $x$ под действием $\psi_{D}$. Назовем разбиение $D$ древесным, если граф $K_{D}$ есть дерево. Связную компоненту $S$ пространства $R(D)$ назовем $k$-компонентой, если $\psi_{D}(S)$ содержит ровно $k$ клювов. Назовем разбиение $D$ ручным, если зацепление $L$ связно, $\chi(D)=0$ (определение эйлеровой характеристики $\chi(D)$ дано в п. 2.7), всякая связная компонента пространства $R(D)$ есть либо 2-компонента, либо 0-компонента, причем найдется хотя бы одна 0 -компонента.

ЛЕмма 6.1. Если $D$ - ручное разбиение, то $D$ древесно и у пространства разрешения $R(D)$ ровно одна 0-компонента.

ДокАЗАТЕЛЬСТво. Всякому ориентированному отрезку $J \subset L$ с концами вне $\mathrm{Sw}_{L}(D)$ можно поставить в соответствие путь на графе $K_{D}$ - конечную последовательность ребер, у которой начало следующего ребра совпадает с концом предыдушего. Этот путь строится так: точки множества $J \cap \mathrm{Sw}_{L}(D)$ упорядочиваются при помощи ориентации на $J$; переходя к их образам в $\Sigma$, мы получаем последовательность точек из $\mathrm{Sw}(D)$, ей соответствует последовательность ребер графа $K_{D}$. Пользуясь этой конструкцией, легко проверить, что из связности узла $L$ вытекает связность графа $K_{D}$. Условие $\chi(D)=0$ означает, что число ребер графа $K_{D}$ равно числу 2-компонент пространства $R(D)$. Поэтому его эйлерова характеристика $\chi\left(K_{D}\right)$ равна числу 0-компонент и потому $\chi\left(K_{D}\right) \geqslant 1$. Но из связности графа $K_{D}$ следует, что $\chi\left(K_{D}\right) \leqslant 1$. Значит, $K_{D}$ - дерево и у разрешения $R(D)$ ровно одна 0 -компонента.

Пусть разбиение $D$ древесно. Если $\mathrm{Sw}(D)$ непусто, то узел $L$ делится точками множества $\mathrm{Sw}_{L}(D)$ на части, гомеоморфные отрезку. Пусть $S$ - компонента пространства $R(D)$. Обозначим через $S_{L} \subset L$ объединение частей, проецируюшихся в $\psi_{D}(S) \subset$ $\Sigma\left(\right.$ если $\mathrm{Sw}(D)$ пусто и $S=R(D)$, то полагаем $\left.S_{L}=L\right)$. Назовем компоненту $S$ ииклической, если для любой такой пары точек $y_{1}, y_{2} \in \partial S_{L}$, что $\sigma\left(y_{1}\right)=\sigma\left(y_{2}\right)$, найдется такой отрезок $J \subset L$ с концами в точках $y_{1}$ и $y_{2}$, что $J \cap S_{L}=\left\{y_{1}, y_{2}\right\}$ (т.е. говоря нестрого, $L$ превращается в $S$ после стягивания в точку каждого из таких отрезков).

ЛЕмма 6.2. Пусть $L \subset J^{1}\left(S^{1}\right)-\sigma$-общее лежсандрово зацепление, $D$ - древесное разбиение его фронта $\Sigma \subset J^{0}\left(S^{1}\right)$. Тогда $L$ связно и всякая компонента пространства $R(D)$ является ииклической. Кроме того, для любой переключающей точки $x \in \mathrm{Sw}(D)$ и любой компоненты $S^{*} \subset R(D)$ множество $S_{L}^{*}$ челиком содержится в одном из двух отрезков, на которые прообразы точки $х$ делят узелL. 
ДокАЗАТЕльство. Доказательство проводим индукцией по числу $k=\#(\operatorname{Sw}(D))$. При $k=0$ утверждение очевидно. Предположим, что утверждение доказано для всех древесных разбиений $D_{0}$ с \# $\left(\mathrm{Sw}\left(D_{0}\right)\right)=k-1$. Пусть $D$ древесно, \# $(\mathrm{Sw}(D))=k>0$. Тогда у дерева $K_{D}$ есть по меньшей мере две вершины валентности 1 . Следовательно, найдется такая компонента $S \subset R(D)$, что $\psi_{D}(S)$ содержит ровно одну переключающую точку разбиения $D$. Обозначим эту точку через $x_{S}$, ее прообразы в $L$ через $z_{1}, z_{2}$.

Рассмотрим такое разбиение $D_{0}$ фронта $\Sigma$, что $\mathrm{Sw}\left(D_{0}\right)=\mathrm{Sw}(D) \backslash\left\{x_{S}\right\}$. Поскольку граф $K_{D}$ получается из $K_{D_{0}}$ стягиванием одного ребра в точку, разбиение $D_{0}$ древесно. Покажем, что утверждения леммы для разбиения $D$ вытекают из утверждений для $D_{0}$, верных по предположению индукции. Действительно, это вытекает из следуюшего описания множеств $S_{L}$, где $S$ - компонента $R(D)$ : одно из них есть отрезок $J_{S} \subset L$ с концами в точках $z_{1}, z_{2}$, не содержаший других точек множества $\mathrm{Sw}_{L}(D)$; еще одно есть $S_{L}^{\prime} \backslash J_{S}$, где $S^{\prime}$ - компонента пространства $R\left(D_{0}\right)$, содержащая прообразы точки $x_{S}$; остальные совпадают с множествами вида $S_{L}^{\prime \prime}$, где $S^{\prime \prime}$ - некоторая компонента пространства $R\left(D_{0}\right)$.

6.3. Ручные разбиения и отрезки на лежандровом узле. Пусть $\Lambda$ - отрезок, $\gamma: \Lambda \rightarrow \Sigma$-сечение фронта $\Sigma, y$-внутренняя точка отрезка $\Lambda$. Назовем полусечениями (по отношению к точке $y$ ) два отображения, получающиеся из $\gamma$ ограничением на отрезки, на которые $y$ делит $\Lambda$. Назовем правылм полусечением то из них, которое кончается в правом клюве фронта $\Sigma$, левым полусечением - то, которое кончается в левом клюве.

ЛЕмма 6.3. Пусть $D$ - ручное разбиение $\sigma$-общего лежандрова узла $L \subset J^{1}\left(S^{1}\right)$, $S^{0}$ - 0-компонента разрешения $R(D)$. Тогда всякая точка $x \in \mathrm{Sw}(D)$ единственным образом определяет следующие облекты.

(1) Отрезок $J_{x}^{*}(D) \subset L$ с концами в прообразах точки $x$, не содержащий внутренних точек множества $S_{L}^{0}$.

(2) Сечение $\gamma_{x}: \Lambda \rightarrow \sigma(L)$ разбиения $D$, где $\Lambda$ - отрезок, такое, что его образ содержит точку $x$ и является подмножеством множества $\sigma\left(J_{x}^{*}(D)\right)$; обозначим через $\gamma_{x}^{r}$ его правое полусечение относительно прообраза точки $x$ в $\Lambda$, через $\gamma_{x}^{l}-$ левое полусечение.

(3) Непересекающиеся отрезки $J_{x}^{r}(D), J_{x}^{l}(D) \subset J_{x}^{*}(D)$, конщы которых отображаются проекцией $\sigma$ в концы полусечений $\gamma_{x}^{r}, \gamma_{x}^{l}$ соответственно.

При этом различные отрезки $J_{x}^{*}(D), J_{x^{\prime}}^{*}(D)$ или попарно не пересекаются, или один из них есть подмножество внутренности другого. Отрезки $J_{x}^{*}(D)$ для переключающих точек $x$, принадлежащих сечению $\psi_{D}\left(S^{0}\right)$, попарно не пересекаются и $L$ есть обғединение всех таких отрезков и множества $S_{L}^{0}$. Сечения $\gamma_{x}$, соответствующие разным переключающим точкам, различны, и всякий клюв является концом ровно одного из них. Точки отрезков $J_{x}^{r}(D), J_{x}^{l}(D)$, близкие $\kappa$ их концам, переводятся проекцией $\sigma$ в точки полусечений $\gamma_{x}^{r}, \gamma_{x}^{l}$ соответственно.

ДоКАЗАТЕЛЬСтво. Разбиение $D$ древесно по лемме 6.1. По лемме 6.2 множество $S_{L}^{0}$ целиком содержится в одном из двух отрезков, на которые прообразы точки $x$ делят узел $L$, обозначим его $I_{x}^{*}(D)$. Обозначим другой отрезок через $J_{x}^{*}(D)$. Отре- 
зок $J_{x}^{*}(D) \subset L$ не содержит внутренних точек множества $S_{L}^{0}$ по построению. Концы отрезка $J_{x^{\prime}}^{*}(D) \subset L$, где $x^{\prime} \neq x$, принадлежат множеству $S_{L}^{\prime}$, где $S^{\prime}$ - некоторая компонента пространства $R(D)$. Из леммы 6.2 следует, что эти концы одновременно принадлежат одному из отрезков $I_{x}^{*}(D), J_{x}^{*}(D)$. Поэтому отрезки $J_{x}^{*}(D), J_{x^{\prime}}^{*}(D)$ либо не пересекаются, либо один из них есть подмножество внутренности другого, либо их объединение есть $L$. Но последнее невозможно, так как внутренние точки непустого множества $S_{L}^{0}$ не принадлежат ни одному из них.

Пусть $x$ - точка множества $\mathrm{Sw}_{0}(D)$, состоящего из переключающих точек, принадлежаших сечению $\psi_{D}\left(S^{0}\right)$. Тогда отрезок $J_{x}^{*}(D) \subset L$ есть замыкание связной компоненты дополнения к $S_{L}^{0}$ в $L$ (так как его концы принадлежат $S_{L}^{0}$ ). Отсюда вытекает, что отрезки $J_{x}^{*}(D) \subset L$, отвечаюшие разным $x \in \mathrm{Sw}_{0}(D)$, попарно не пересекаются и $L$ является объединением всех таких отрезков и множества $S_{L}^{0}$.

Из древесности разбиения $D$ следует, что точка $x \in \mathrm{Sw}(D)$ принадлежит образам двух различных сечений. Обозначим их $\gamma_{1}, \gamma_{2}$. Рассмотрим такие компоненты $S^{1}, S^{2}$ пространства $R(D)$, что образ сечения $\gamma_{i}$ содержится в $\psi_{D}\left(S^{i}\right)$. По лемме 6.2 каждое из множеств $S_{L}^{i}$ является подмножеством одного из отрезков $I_{x}^{*}(D), J_{x}^{*}(D)$. Для каждого из прообразов точки $x$ в $L$ одна его малая полуокрестность содержится в $S_{L}^{1}$, а другая в $S_{L}^{2}$. Поэтому найдется ровно одно такое $i \in\{1,2\}$, что отрезок $J_{x}^{*}(D)$ содержит множество $S_{L}^{i}$. Положим $\gamma_{x}=\gamma_{i}, S^{x}=S^{i}$. Тогда образ сечения $\gamma_{x}$ является подмножеством множества $\sigma\left(J_{x}^{*}(D)\right)$. Из того, что $S_{L}^{i} \subset J_{x}^{*}(D)$, следует, что $S^{i} \neq S^{0}$. Поэтому $S^{i}$ является 2-компонентой, и, следовательно, область определения сечения $\gamma_{x}$ есть отрезок.

Докажем, что сечения $\gamma_{x}, \gamma_{x^{\prime}}$, соответствующие разным переключающим точкам, различны. Предположим противное. Тогда $S^{x}=S^{x^{\prime}}$, пересечение отрезков $J_{x}^{*}(D)$, $J_{x^{\prime}}^{*}(D)$ содержит множество $S_{L}^{x}$ и, следовательно, непусто. Поэтому один из этих отрезков есть подмножество внутренности другого. Но это противоречит тому, что концы обоих отрезков содержатся в $S_{L}^{x}$. Значит, $\gamma_{x} \neq \gamma_{x^{\prime}}, S^{x} \neq S^{x^{\prime}}$.

Поскольку разбиение $D$ ручное, число его переключаюших точек равно числу 2-компонент разрешения $R(D)$. С ледовательно, отображение $x \mapsto S^{x}$ задает взаимно однозначное соответствие между переключаюшими точками и 2-компонентами. Значит, для всякого клюва фронта $\sigma(L)$ найдется ровно одна такая переключающая точка $x$, что прообраз этого клюва в $R(D)$ принадлежит компоненте $S^{x}$. Поэтому $x-$ единственная переключающая точка, для которой сечение $\gamma_{x}$ заканчивается в этом клюве.

Обозначим через $Z_{L}^{r}$ (соответственно $Z_{L}^{l}$ ) замыкание подмножества $L$, состоящее из всех таких точек $z$, что $\sigma(z)$ - неособая точка фронта $\sigma(L)$, принадлежашая образу полусечения $\gamma_{x}^{r}$ (соответственно $\gamma_{x}^{l}$ ). Пусть точка $x^{\prime} \in \mathrm{Sw}(D)$ принадлежит образу полусечения $\gamma_{x}^{r}$, обозначим множество всех таких точек через $\mathrm{Sw}_{x}^{r}$. Обозначим через $J_{x}^{r}(D)$ объединение множества $Z_{L}^{r}$ и всех таких отрезков $J_{x^{\prime}}^{*}$, что $x^{\prime} \in \mathrm{Sw}_{x}^{r}$. Концы отрезка $J_{x^{\prime}}^{*}$ принадлежат множеству $Z_{L}^{r}$, а его внутренние точки не принадлежат множеству $S_{L}^{x}$ по лемме 6.2 . Граничные точки множества $Z_{L}^{r}$ - это в точности концы отрезков $J_{x}^{*}$, где $x^{\prime} \in \mathrm{Sw}_{x}^{r}$, и точки, отображаюшиеся проекцией $\sigma$ в конщы полусечения $\gamma_{x}^{r}$. Отсюда следует, что множество $J_{x}^{r}(D)$ является отрезком и требования леммы, касаюшиеся его концов и их окрестностей, вьполнены. Отрезок $J_{x}^{r}(D)$ является подмножеством отрезка $J_{x}^{*}(D)$, поскольку $Z_{L}^{r} \subset J_{x}^{*}(D)$ и каждый из отрезков $J_{x^{\prime}}^{*}$, где 
$x^{\prime} \in \mathrm{Sw}_{x}^{r}$, есть подмножество отрезка $J_{x}^{*}(D)$ по лемме 6.2 (так как конщы отрезка $J_{x^{\prime}}^{*}$ являются внутренними точками отрезка $\left.J_{x}^{*}(D)\right)$. Аналогичным образом определяется отрезок $J_{x}^{l}(D)$ и проверяются его свойства. Отрезки $J_{x}^{r}(D)$ и $J_{x}^{l}(D)$ не пересекаются, так как в противном случае клюв, являюшийся концом правого полусечения, принадлежал бы также и левому, что невозможно.

\section{$\S 7$. Теоремы Гурвица для фронтов}

7.1. Обобщенное отображение кривизны. Пусть $\lambda \in \mathbb{R}$. Рассмотрим 1-фором $\omega=d q, \beta_{\lambda}=d p+\lambda u d q$ на $J^{1}\left(S^{1}\right)$. Для лежандрова зацепления $L \in J^{1}\left(S^{1}\right)$ определим отображение $\operatorname{Curv}_{L, \lambda}: L \rightarrow \mathbb{R P}^{1}=\mathbb{R} \cup\{\infty\}$, полагая $\operatorname{Curv}_{L, \lambda}(z)=\left[\left.\omega\right|_{L}(z)\right.$ : $\left.\left.\beta_{\lambda}\right|_{L}(z)\right]$ (т.е. $\operatorname{Curv}_{L, \lambda}(z)=\infty$, если $\left.\beta_{\lambda}\right|_{L}(z)=0$ и и $\left.\omega\right|_{L}(z)=\left.\operatorname{Curv}_{L, \lambda}(z) \beta_{\lambda}\right|_{L}(z)$, если $\left.\beta_{\lambda}\right|_{L}(z) \neq 0$ ). Это определение корректно (т.е. 1-формы $\left.\beta_{\lambda}\right|_{L}(z),\left.\omega\right|_{L}(z) \in T_{z}^{*} L$ не обращаются в нуль одновременно), так как 1-формы $\alpha=d u-p d q, \beta_{\lambda}$ и $\omega$ линейно независимы в каждой точке. Это определение при $\lambda=1$ совпадает с определением отображения $\operatorname{Curv}_{L}$, данньм в п. 0.2. Определим отображение $F_{L, \lambda}: L \rightarrow \mathbb{R} P^{1}=\mathbb{R} \cup\{\infty\}$. Положим $F_{L, \lambda}(y)=\infty$, если $y \notin G_{L}$. В окрестности точки $y \in G_{L}$ лежандрово зацепление $L$ совпадает с 1 -графиком $j^{1} f$ некоторой функции $f: U \rightarrow \mathbb{R}, U \subset S^{1}$. Положим $F_{L, \lambda}(y)=f^{\prime \prime}\left(q_{y}\right)+\lambda f\left(q_{y}\right)$, где $q_{y}-q$-координата точки $y$. Рассуждая как в доказательстве леммы 1.1, можно показать, что $F_{L, \lambda}=1 / \operatorname{Curv}_{L, \lambda}$.

7.2. Точки Штурма и точки Арнольда. Точку $z \in L$ назовем $\lambda$-точкой Штурма, если $F_{L, \lambda}(z)=0$. Критические точки отображения $F_{L, \lambda}: L \rightarrow \mathbb{R P}^{1}$ назовем $\lambda$-точками Арнольда. Для любого $\lambda \in \mathbb{R} \quad \lambda$-точки Штурма принадлежат $G_{L}$, так как множество $G_{L}$ состоит из всех таких $z \in L$, что $\left.\omega\right|_{L}(z) \neq 0$. Точка $z=\left(p_{0}, q_{0}, u_{0}\right) \in L$ назьвается критической точкой зацепления $L$, если $p_{0}=0$.

7.3. Непрерывное сечение фронта. Пусть $\mathscr{L}_{1}$ - связная компонента пространства лежандровых узлов в $J^{1}\left(S^{1}\right)$, содержашая нулевое сечение расслоения $J^{1}\left(S^{1}\right) \rightarrow S^{1}$. Рассмотрим пространство $\mathscr{L}_{1}^{+}$лежандровых зацеплений такое, что всякое $L^{+} \in \mathscr{L}_{1}^{+}$получается из некоторого $L \in \mathscr{L}_{1}$ добавлением такой компоненты $V_{c}=\{p=0, u=c\}$, что значение координаты $u$ на $L$ меньше $c$.

Естественная проекция $\mathscr{L}_{1}^{+} \rightarrow \mathscr{L}_{1}$ - расслоение со стягиваемым слоем. Компонента $\mathscr{L}_{1}^{+}$содержит зацепление $V_{0} \cup V_{1}$, фронт которого допускает ровно одну, положительную, псевдоинволюцию $\mathrm{P}_{0}$. Согласно утверждению 2.6 , фронт $\sigma$-общего зацепления из $\mathscr{L}_{1}^{+}$допускает ровно одну положительную псевдоинволюцию, при этом ее эйлерова характеристика равна нулю. Рассмотрим отображение $L^{+} \mapsto \mathscr{P}\left(L^{+}\right)$из теоремы 5.1 , сопоставляющее зацеплению $L^{+}$псевдоинволюцию фронта $\sigma\left(L^{+}\right)$. Определим отображение $\mathscr{H}: \mathscr{L}_{1} \rightarrow C^{0}\left(S^{1}\right)$ следуюшим образом. Пусть $L \in \mathscr{L}_{1}$, возьмем $L^{+}=L \cup V_{c} \subset \mathscr{L}_{1}^{+}$и определим $\mathscr{H}(L)$ как функцию, график которой есть $\mathscr{P}\left(L^{+}\right)\left(V_{c}\right)$. Легко видеть, что отображение $\mathscr{H}$ определено корректно (не зависит от $c$ ). В силу теоремы 5.1 справедливо следуюшее утверждение.

УТВЕРЖДЕНИЕ 7.1. Отображение $\mathscr{H}: \mathscr{L}_{1} \rightarrow C^{0}\left(S^{1}\right)$ непрерывным образом сопоставляет лежсандрову узлу $L$ функиию $\mathscr{H}(L)$, график $\Gamma_{\mathscr{H}(L)}$ которой является подмножеством фронта $\sigma(L)$. 
7.4. Канонический масловский потенциал. Рассмотрим накрытие $\mathscr{M}_{1}$ над $\mathscr{L}_{1}$, слой которого над $L \in \mathscr{L}_{1}$ - это целочисленные масловские потенщиалы на $L$.

Лемма 7.2. У накрытия $\mathscr{M}_{1}$ есть единственное непрерывное сечение $L \rightarrow \mu_{L}$ такое, что $\mu_{V_{0}}=\{0\}$. Для любого общего узла $L \in \mathscr{L}_{1}$ значение масловского потенциала $\mu_{L}$ на прообразе всякой неособой точки фронта $\sigma(L)$, содержащейся в Г Н $(L)$, равно нулю. Для любого прообраза $L^{+} \in \mathscr{L}_{1}^{+}$узла $L$ псевдоинволюиия $\mathscr{P}\left(L^{+}\right)$масловская относительно масловского потенииала, равного $\mu_{L}$ на $L u$ единиче на дополнительной компоненте.

ДокАЗАТЕльство. Пусть $\mu$ - масловский потенциал на $V_{0} \cup V_{1}$ такой, что $\mu\left(V_{0}\right)=0, \mu\left(V_{1}\right)=1$. Для доказательства первого утверждения леммы достаточно проверить, что всякий масловский потенциал $\mu^{\prime}$, полученньй из $\mu$ непрерьвным продолжением вдоль петли в $\mathscr{L}_{1}^{+}$, совпадает с $\mu$. Это вытекает из теоремы 2.5 и того, что $\mu^{\prime}\left(V_{1}\right)=1$. Оставшиеся утверждения немедленно следуют из теоремы 2.5 .

7.5. Три обобщения теоремы Гурвица. Пусть $L \in \mathscr{L}_{1}$. Обозначим через $N_{\lambda}^{\mathrm{S}}(L)$ множество $\lambda$-точек Штурма на $L$, значение масловского потенциала $\mu_{L}$ в которых равно нулю. Обозначим через $N_{\lambda}^{\mathrm{A}}(L)$ множество $\lambda$-точек Арнольда на $L$.

Определим скалярное произведение функций на $S^{1}$ как $L^{2}$-спаривание: $\langle f, g\rangle=$ $\int_{S^{1}} f(q) g(q) d q$. Следуюшие три теоремы являются обобшениями теоремы Гурвица и сводятся к ней в случае, когда узел $L$ есть 1-график гладкой функции на $S^{1}$.

ТеОРема 7.3. Пусть $L \in \mathscr{L}_{1}$ - такой лежандров узел, что функция $\mathscr{H}(L)$ ортогональна $2 k+1$ функииям $1, \cos q, \sin q, \ldots, \cos k q, \sin k q$. Тогда для любого $\lambda \in \mathbb{R}$ справедливо неравенство $\#\left(N_{\lambda}^{\mathrm{S}}(L)\right) \geqslant 2 k+2, u \#\left(N_{\lambda}^{\mathrm{S}}(L)\right) \geqslant 2 k+4$ при $\lambda=(k+1)^{2}$.

Теорема 7.4. Пусть $L \in \mathscr{L}_{1}$ - такой лежандров узел, что функция $\mathscr{H}(L)$ ортогональна $2 k$ функииям $\cos q, \sin q, \ldots, \cos k q, \sin k q$. Тогда для любого $\lambda \in \mathbb{R}$ справедливо неравенство \# $\left(N_{\lambda}^{\mathrm{A}}(L)\right) \geqslant 2 k+2, u \#\left(N_{\lambda}^{\mathrm{A}}(L)\right) \geqslant 2 k+4$ при $\lambda=$ $(k+1)^{2}$.

Теорема 7.5. Пусть $L \in \mathscr{L}_{1}$ - такой лежандров узел, что функция $\mathscr{H}(L)$ ортогональна $2 k$ функииям $\cos q, \sin q, \ldots, \cos k q, \sin k q$. Тогда на L найдется по меньшей мере $2 k+2$ критические точки.

7.6. Замечание о нулях старших производных. Для всякой гладкой функции на окружности и любого натурального $n$ ее $n$-я производная имеет по меньшей мере два нуля. Можно спросить, верны ли аналоги этого утверждения для лежандровых узлов из компоненты $\mathscr{L}_{1}$.

Пусть $L \in J^{1}\left(S^{1}\right)$ - лежандрово зацепление. Определим функцию $h_{n, L}: G_{L} \rightarrow \mathbb{R}$. Пусть $y \in G_{L}, f$ - гладкая функщия, 1-график которой совпадает с $L$ в окрестности точки $y$. Положим $h_{n, L}(y)=f^{(n)}\left(q_{y}\right)$, где $q_{y}-q$-координата точки $y$. Обозначим через $Q_{n}(L)$ множество нулей функции $h_{n, L}$. Обозначим через $\bar{Q}_{n}(L)$ множество, состоящее из таких точек $y \in L$, что найдется сколь угодно $C^{\infty}$-близкое к $L$ лежандрово зацепление $L^{\prime}$, содержашее точку $y^{\prime} \in Q_{n}\left(L^{\prime}\right)$, близкую к точке $y$. Тогда для любого $n$ множество $Q_{n}(L) \subset \bar{Q}_{n}(L), \bar{Q}_{1}(L)$ есть множество критических точек зацепления $L$, $Q_{2}(L)=\bar{Q}_{2}(L)$ есть множество 0-точек Штурма зацепления $L, \bar{Q}_{3}(L)$ есть множество 0 -точек Арнольда зацепления $L$. 
Из сформулированных вьше теорем вытекает, что для любого $L \in \mathscr{L}_{1}$ каждое из множеств $\bar{Q}_{1}(L), \bar{Q}_{2}(L), \bar{Q}_{3}(L)$ содержит по меньшей мере две точки. Несложно показать, что для любого нечетного $n \geqslant 3$ и любого $L \in \mathscr{L}_{1}$ множество $\bar{Q}_{n}(L)$ также содержит по меньшей мере две точки. (Дадим набросок доказательства этого утверждения для $\sigma$-общего лежандрова узла $L$, не являющегося 1 -графиком гладкой функции. Из $m(L)=0$ следует, что найдется по меньшей мере два таких отрезка $J \subset L$, что концы каждого из них (но не внутренние точки) проектируются в клювы фронта $\sigma(L)$, и кривая $\sigma(J)$ либо входит в оба клюва сверху, либо в оба снизу. Тогда при приближении к одному из концов отрезка $J$ функция $h_{n, L}$ стремится к $+\infty, \mathrm{k}$ другому $-\mathrm{k}-\infty$. Поэтому на отрезке $J$ найдется точка множества $Q_{n}(L)$.) Можно, однако, привести пример такого $\sigma$-общего лежандрова узла $L \in \mathscr{L}_{1}$ (фронт которого имеет два клюва и одну точку самопересечения), что множество $\bar{Q}_{4}(L)$ пусто. Вероятно, подобные примеры можно построить и для четных $n>4$.

\section{§ 8. Доказательства обобщений теоремы Гурвица}

8.1. Назовем лежандрово зацепление $L \subset J^{1}\left(S^{1}\right) \quad \lambda$-общим, если оно $\sigma$-общее, образы $\lambda$-точек Штурма являются неособыми точками фронта $\sigma(L)$ и дифференциал отображения $F_{L, \lambda}$ отличен от нуля в любой $\lambda$-точке Штурма. Докажем утверждение теоремы 7.3 для $\lambda$-общего узла $L$.

Возьмем лежандрово зацепление $L^{+}=L \cup V_{c} \in \mathscr{L}_{1}^{+}$, переходяшее в узел $L \in \mathscr{L}_{1}$ при проекции $\mathscr{L}_{1}^{+} \rightarrow \mathscr{L}_{1}$. Пусть $\mathrm{P}=\mathscr{P}\left(L^{+}\right)$- положительная псевдоинволюция фронта $\sigma\left(L^{+}\right)$. Множество $\mathrm{P}\left(V_{c}\right) \subset \Sigma=\sigma(L)$ есть график $\Gamma_{H_{L}}$ функции $H_{L}=\mathscr{H}(L)$. Обозначим через $Y$ проекцию множества $\mathrm{Sw}(\mathrm{P}) \cap \Gamma_{H_{L}}$ на $S^{1}$. Кусочно-гладкая функция $H_{L}$ является гладкой вне $Y$, в точках множества $Y$ ее правые и левые производные различны. Рассмотрим множество $\mathscr{G}_{Y}$ обобщенных функций (элементов пространства, двойственного к $\left.C^{\infty}\left(S^{1}\right)\right)$, имеющих вид $\varphi=\sum_{s \in Y} b_{\varphi}^{s} \delta_{s}+g_{\varphi}$, где $\delta_{s}-\delta$-функция Дирака с носителем в точке $s, b_{\varphi}^{s}$ - ненулевое число, $g_{\varphi}$ - гладкая функция на $S^{1} \backslash Y$ с регулярными нулями, имеющая ненулевые левые и правые пределы в каждой из точек $s \in Y$. (Более подробно: результат применения обобщенной функции $\varphi$ к функции $f \in C^{\infty}\left(S^{1}\right)$ есть $\left.\sum_{s \in Y} b_{\varphi}^{s} f(s)+\int_{S^{1} \backslash Y} f(q) g(q) d q.\right)$

8.2. Перемены знака. Определим знак обобщенной функции $\varphi \in \mathscr{G}_{Y}$ в точке $q \in S^{1}$ как знак числа $b_{\varphi}^{q}$ при $q \in Y$ и как знак числа $g_{\varphi}(q)$ при $q \notin Y$. Будем говорить, что функция $\varphi \in \mathscr{G}_{Y}$ меняет знак справа (слева) в точке $s \in Y$, если знак числа $b_{\varphi}^{s}$ отличен от знака правого (левого) предела функции $g_{\varphi}$ в точке $s$. Вообще говоря, функция $\varphi$ может менять знак в точке $s \in Y$ дважды. Функция $\varphi$ меняет знак в точке $q \in S^{1} \backslash Y$, если $g_{\varphi}$ меняет знак в точке $q$.

Если узел $L \lambda$-общий, то результат применения дифференциального оператора $D_{\lambda}=\frac{d^{2}}{d q^{2}}+\lambda$ к функции $H_{L}$ есть обобщенная функция из множества $\mathscr{G}_{Y}$, и $g_{D_{\lambda} H_{L}}(q)=H_{L}^{\prime \prime}(q)+\lambda H_{L}(q)$ при $q \in S^{1} \backslash Y$. Рассуждая как в доказательстве леммы 1.1 , легко увидеть, что если $D_{\lambda} H_{L}\left(q_{0}\right)=0$ при $q_{0} \notin Y$, то $F_{L, \lambda}\left(z_{0}\right)=0$, где $z_{0} \in L$ - такая точка, что $\sigma\left(z_{0}\right)=\left(q_{0}, H_{L}\left(q_{0}\right)\right)$. Из леммы 7.2 следует, что $z_{0} \in N_{\lambda}^{\mathrm{S}}(L)$. 


\section{3. Теорема Гурвица для обобщенных функций.}

ЛЕмма 8.1. Если обобщенная функиия $\varphi \in \mathscr{G}_{Y}$ аннулирует функиии $1, \cos q$, $\sin q, \ldots, \cos l q, \sin l q$, то она меняет знак по меньшей мере $2 l+2$ раза.

ДоказАТЕльство. Обозначим через $Y_{i}, i \in\{1,2\}$, множество точек окружности, в которых функция $D_{\lambda} H_{L}$ меняет знак ровно $i$ раз; заметим, что $Y_{2} \subset Y$. Предположим, что

$$
2 l^{\prime}=\#\left(Y_{1}\right)+2 \#\left(Y_{2}\right)<2 l+2 .
$$

Перемножая подходящие тригонометрические многочлены степени 1 , построим тригонометрический многочлен $Q$ степени $l^{\prime}$, которьй обрашается в нуль в точности на множестве $Y_{1} \cup Y_{2}$, меняет знак в точках множества $Y_{1}$ и не меняет знака в точках множества $Y_{2}$. Изменяя знак функции $Q$, если необходимо, можно считать, что знаки функций $D_{\lambda} H_{L}$ и $Q$ совпадают в точках множества $S^{1} \backslash\left(Y_{1} \cup Y_{2}\right)$. Докажем, что

$$
\left\langle D_{\lambda} H_{L}, Q\right\rangle=\sum_{s \in Y} b_{D_{\lambda} H_{L}}^{s} Q(s)+\int_{S^{1} \backslash Y} Q(q) g_{D_{\lambda} H_{L}}(q) d q>0
$$

Действительно, в силу выбора многочлена $Q$, второе слагаемое положительно, а первое равно

$$
\sum_{s \in Y \backslash\left(Y_{1} \cup Y_{2}\right)} b_{D_{\lambda} H_{L}}^{s} Q(s) \geqslant 0 .
$$

Значит, $D_{\lambda} H_{L}$ не аннулирует $Q$. Противоречие.

8.4. Применение леммы 8.1. Пространство $\mathrm{T}_{k}$ тригонометрических многочленов степени не вьше $k$ инвариантно относительно действия дифференциального оператора $D_{\lambda}$. Поскольку оператор $D_{\lambda}$ самосопряжен, из ортогональности $H_{L}$ пространству $\mathrm{T}_{k}$ следует, что $D_{\lambda} H_{L}$ аннулирует функции из $T_{k}$. Если $\lambda=(k+1)^{2}$, то функции $\sin (k+1) q, \cos (k+1) q$ лежат в ядре оператора $D_{\lambda}$, а следовательно, аннулируются обобщенной функцией $D_{\lambda} H_{L}$. Значит, в условиях теоремы 7.3 обобщенная функция $D_{\lambda} H_{L}$ (для $\lambda$-общего $L$ ) всегда имеет не менее $2 k+2$ перемен знака, а при $\lambda=(k+1)^{2}$ не менее $2 k+4$ перемен знака.

Для каждой перемены знака обобщенной функции $D_{\lambda} H_{L}$, происходящей в точке множества $Y$, мы найдем точку множества $N_{\lambda}^{\mathrm{S}}(L)$. Найденные таким образом $\lambda$-точки Штурма будут различными и отличными от точек, соответствующих нулям функшии $g_{D_{\lambda} H_{L}}$. Это завершит доказательство теоремы 7.3 для случая $\lambda$-общего узла.

8.5. Псевдоинволюции и ручные разбиения. Пусть $L^{+}=L \cup V_{c}$ есть прообраз $L$ в $\mathscr{L}_{1}^{+}$при проекции $\mathscr{L}_{1}^{+} \rightarrow \mathscr{L}_{1}$. Псевдоинволюция $\mathrm{P}^{+}=\mathscr{P}\left(L^{+}\right)$порождает разбиение $D_{\mathrm{P}+}$ фронта $\sigma(L) \cup \sigma\left(V_{c}\right)$. Обозначим через $D_{L}$ разбиение фронта $\Sigma=\sigma(L)$, полученное из $D_{\mathrm{P}+}$ выбрасыванием сечения $\sigma\left(V_{c}\right)$. Разбиение $D_{L}$ не зависит от выбора $L^{+}$. Определение ручного разбиения было дано в п. 6.2.

Лемма 8.2. Разбиение $D_{L}$ ручное. 
ДокАЗАТЕльСтво. Если $\mathrm{P}$ - псевдоинволющия $\sigma$-общего фронта $\Sigma^{\prime} \subset J^{0}\left(S^{1}\right)$, то всякая компонента пространства разрешения $R\left(D_{\mathrm{P}}\right)$ есть либо 2-компонента, либо 0-компонента. Это следует из того, что если у двух сечений разбиения $D_{\mathrm{P}}$ совпадают правые концы, то и левые тоже совпадают. Поэтому у пространства $R\left(D_{L}\right)$ всякая компонента есть либо 2-компонента, либо 0-компонента. У пространства $R\left(D_{L}\right)$ есть 0 -компонента, проецирующаяся в сечение $\Gamma_{H_{L}}$. Из теоремы 2.5 следует, что $\chi\left(D_{L}\right)=$ $\chi\left(\mathrm{P}^{+}\right)=0$.

8.6. Поиск нулей $F_{L, \lambda}$ для обобщенных перемен знака. График $\Gamma_{H_{L}}-$ образ единственной (согласно лемме 6.1) 0-компоненты $S^{0}$ пространства $R\left(D_{L}\right)$ под действием отображения $\psi_{D_{L}}$. Для каждой точки $s \in Y$ обозначим через $\hat{s}=\left(s, H_{L}(s)\right) \in$ $\mathrm{Sw}_{0}\left(D_{L}\right)=\Gamma_{H_{L}} \cap \mathrm{Sw}\left(D_{L}\right)$ соответствуюшую точку излома графика. Поскольку разбиение $D_{L}$ ручное, лемма 6.3 по точке $\hat{s}$ строит отрезки $J_{\hat{s}}^{r}\left(D_{L}\right), J_{\hat{s}}^{l}\left(D_{L}\right) \subset L$.

Лемма 8.3. Пусть $s \in Y$. Если обобщенная функиия $D_{\lambda} H_{L}$ меняет знак слева в точке $s$, то на отрезке $J_{\hat{s}}^{r}\left(D_{L}\right)$ найдется точка множества $N_{\lambda}^{\mathrm{S}}(L)$. Если $D_{\lambda} H_{L}$ меняет знак справа в точке $s$, то на отрезке $J_{\hat{s}}^{l}\left(D_{L}\right)$ найдется точка множества $N_{\lambda}^{\mathrm{S}}(L)$.

Пользуясь леммой 8.3, каждой перемене знака обобшенной функции $D_{\lambda} H_{L}$ в точке $s \in Y$ можно поставить в соответствие точку множества $N_{\lambda}^{\mathrm{S}}(L)$. Согласно лемме 6.3 , точки множества $N_{\lambda}^{\mathrm{S}}(L)$, отвечаюшие различньм переменам знака, различны и не лежат на $\Gamma_{H_{L}}$. Следовательно, число точек множества $N_{\lambda}^{\mathrm{S}}(L)$ не меньше числа перемен знака обобщенной функции $D_{\lambda} H_{L}$, т.е. утверждение теоремы 7.3 справедливо для $\lambda$-обшего узла. Прежде чем доказьвать лемму 8.3 , сформулируем и докажем лемму 8.4, которая будет использована также в доказательстве леммы 8.12.

8.7. Индекс Маслова и кривизна. Пусть $e: \mathbb{R} \rightarrow \mathbb{R P}^{1}$ - универсальное накрытие. Зафиксируем функцию $\mathrm{i}_{e}: \mathbb{R} \backslash e^{-1}(\{\infty\}) \rightarrow \mathbb{Z}$, которая постоянна на каждом из интервалов, из которых состоит $\mathbb{R} \backslash e^{-1}(\{\infty\})$, и удовлетворяет следующему условию: для любой точки $y \in e^{-1}(\{\infty\})$ и любых двух близких к ней точек $x_{1}, x_{2} \in \mathbb{R} \backslash e^{-1}(\{\infty\})$ таких, что $e\left(x_{1}\right)<0, e\left(x_{2}\right)>0\left(e\left(x_{1}\right), e\left(x_{2}\right) \in \mathbb{R} P^{1} \backslash\{\infty\}\right)$, справедливо равенство $\mathrm{i}_{e}\left(x_{2}\right)=\mathrm{i}_{e}\left(x_{1}\right)+1$. Пусть $l:[0, T] \rightarrow L-$ такой путь (непрерьвное отображение), что $l(0), l(T) \in G_{L}$. Рассмотрим поднятие $\widetilde{l}_{\lambda}$ отображения $F_{L, \lambda} \circ l:[0, T] \rightarrow \mathbb{R P}^{1}$ - такое непрерывное отображение $\widetilde{l}_{\lambda}:[0, T] \rightarrow \mathbb{R}$, что $e \circ \widetilde{l}_{\lambda}=F_{L, \lambda} \circ l$. Положим $\mathrm{i}_{\lambda}(l)=\mathrm{i}_{e}\left(\widetilde{l}_{\lambda}(T)\right)-\mathrm{i}_{e}\left(\widetilde{l}_{\lambda}(0)\right)$. Легко видеть, что число $\mathrm{i}_{\lambda}(l)$ не зависит от выбора поднятия. Индекс Маслова $m(l)$ пути $l$ был определен в п. 2.5 .

Лemma 8.4. $\mathrm{i}_{\lambda}(l)=m(l)$.

Из леммы 8.4 также вытекает, что число Маслова лежандрова узла $L$ совпадает со степенью отображения $F_{L, \lambda}$ при некотором выборе ориентаций.

Лемма 8.5. Пусть $x-$ клюв фронта $\Sigma=\sigma(L)$. Тогда для любой точки $y \in$ $G_{\Sigma}$, достаточно близкой $\kappa x$, значение функиии $F_{L, \lambda}$ на прообразе точки у в $L$ положительно, если у принадлежит верхней из ветвей фронта $\Sigma$, входящих в клюв, и отрицательно, если она принадлежит нижней. 
ДокАЗАТЕльство. Утверждение вытекает из определения отображения $F_{L, \lambda}$ и того, что вторая производная функции, графиком которой является ветвь, входящая в клюв, при подходе к клюву стремится к $+\infty$ для верхней ветви, и к $-\infty$ для нижней.

ДокАЗАТЕЛЬСТво ЛЕммы 8.4. Достаточно доказать утверждение для случая, когда $l:[0, T] \rightarrow L-$ гладкий путь общего положения. Обозначим через $l_{t}$ путь $l_{t}=$ $\left.l\right|_{[0, t]}:[0, t] \rightarrow L$. В силу леммы 8.5 числа $\mathrm{i}_{\lambda}\left(l_{t}\right)$ и $m\left(l_{t}\right)$ меняются на одинаковую величину, когда $t$ проходит через такое значение $t_{0}$, что $l\left(t_{0}\right) \in L \backslash G_{L}$. Это завершает доказательство.

Лемма 8.6. Пусть $L \subset J^{1}\left(S^{1}\right)$ - лежсандрово зачепление, $l:[0, T] \rightarrow L$-такой nуть, что $l(0), l(T) \in G_{L}$. Если знаки чисел $F_{L, \lambda}(l(0)), F_{L, \lambda}(l(T))$ различньи и $m(l)=0$, то найдется такое $t_{1} \in[0, T]$, что $F_{L, \lambda}\left(l\left(t_{1}\right)\right)=0$ u $m\left(l_{1}\right)=0$, где $l_{1}-$ ограничение пути $l$ на отрезок $\left[0, t_{1}\right]$.

ДокаЗАтельство. Рассмотрим отрезок $Z \subset \mathbb{R} \subset \mathbb{R P}^{1}$ с конщами в точках $F_{L, \lambda}(l(0)), F_{L, \lambda}(l(T))$. Из условия на знаки следует, что $0 \in Z$. Рассмотрим поднятие $\widetilde{l}_{\lambda}:[0, T] \rightarrow \mathbb{R}$ отображения $F_{L, \lambda} \circ l:[0, T] \rightarrow \mathbb{R P}^{1}$. Из условия $m(l)=0$ и леммы 8.4 вытекает, что точки $\widetilde{l}_{\lambda}(0), \widetilde{l}_{\lambda}(T)$ лежат в одной связной компоненте множества $\mathbb{R} \backslash e^{-1}(\{\infty\})$. Поэтому отрезок $Z^{\prime}$ с конщами в $\widetilde{l}_{\lambda}(0), \widetilde{l}_{\lambda}(T)$ переводится в $Z$ проекщией $e$. Найдется такая точка $r \in Z^{\prime}$, что $e(r)=0 \in \mathbb{R} \mathrm{P}^{1}$. Поскольку точка $r$ лежит между конщами отрезка $Z^{\prime}$, сушествует такое $t_{1} \in[0, T]$, что $\widetilde{l}_{\lambda}\left(t_{1}\right)=r$. Тогда $F_{L, \lambda}\left(l\left(t_{1}\right)\right)=0$. Кроме того, $\mathrm{i}_{e}\left(\widetilde{l}_{\lambda}(0)\right)=\mathrm{i}_{e}\left(\widetilde{l}_{\lambda}\left(t_{1}\right)\right)$, поэтому по лемме $8.4 m\left(l_{1}\right)=0$.

8.8. Доказательство леммы 8.3. Докажем утверждение леммы для левой перемены знака (доказательство для правой перемены знака аналогично). Рассмотрим отрезок $J^{r} \subset L$, получаюшийся из отрезка $J_{\hat{s}}^{r}\left(D_{L}\right)$ отбрасьванием малых окрестностей конщов. Обозначим через $a^{r}$ конец отрезка $J^{r}$, близкий к конщу отрезка $J_{\hat{s}}^{r}\left(D_{L}\right)$, проектируюшемуся в клюв, через $x^{r}$ - конец отрезка $J^{r}$, близкий к точке $z^{r}$.

Переключающая точка $\hat{s}$ содержится в образах двух сечений из $D_{L}$. Образ одного из этих сечений есть $\Gamma_{H_{L}}$, другое совпадает с сечением $\gamma_{\hat{s}}: \Lambda \rightarrow \Sigma$, определенным в лемме 6.3. Рассмотрим неособые точки фронта $\Sigma$, близкие к переключающей точке $\hat{s}$. По определению масловского потенциала $\mu_{L}$ он принимает нулевое значение на прообразах в $L$ неособых точек фронта $\Sigma$, принадлежаших графику $\Gamma_{H_{L}}$. Следовательно, $\mu_{L}$ обрашается в нуль на прообразе в $L$ малой окрестности точки $\hat{s}$. Из масловости разбиения $D_{L}$ вытекает, что значение масловского потенциала $\mu_{L}$ равно нулю на прообразах в $L$ неособых точек фронта $\Sigma$, принадлежащих $\gamma_{\hat{s}}(\Lambda)$. Поскольку $\sigma\left(a^{r}\right)$, $\sigma\left(x^{r}\right) \in \gamma_{\hat{s}}(\Lambda)$, значение масловского потенциала $\mu_{L}$ в концах отрезка $J^{r}$ равно нулю. Покажем, что числа $F_{L, \lambda}\left(x^{r}\right)$ и $F_{L, \lambda}\left(a^{r}\right)$ имеют разные знаки. После этого применение леммы 8.6 к пути, параметризуюшему отрезок $J^{r}$, завершит доказательство леммы 8.3 .

Предположим, что обобшенная функция $D_{\lambda} H_{L}$ отрицательна в точке $s$, т.е. график $\Gamma_{H_{L}}$ имеет излом “вверх" в точке $\hat{s}$ (доказательство леммы для случая положительного знака аналогично). Поскольку $D_{\lambda} H_{L}$ меняет знак слева в точке $s$, функция $F_{L, \lambda}$ положительна на некоторой окрестности точки $z^{r}$. В частности, $F_{L, \lambda}\left(x^{r}\right)>0$. Рассмотрим точку $d \in \Gamma_{H_{L}}$ с той же $q$-координатой, что и у точки $\sigma\left(x^{r}\right)$. Из того, что $\Gamma_{H_{L}}$ имеет излом "вверх" в точке $\hat{s}$, следует, что точка $\sigma\left(x^{r}\right)$ лежит выше точки $d$ 
(т.е. ее $u$-координата больше). Из положительности псевдоинволюции $\mathrm{P}^{+}{ }_{\text {вытекает }}$ теперь, что точка $\mathrm{P}^{+}\left(\sigma\left(x^{r}\right)\right)$ лежит вьше точки $\sigma\left(x^{r}\right)$. Поэтому для любой общей точки $y \in \gamma_{\hat{s}}(\Lambda)$ точка $\mathrm{P}^{+}(y)$ расположена вьше точки $y$. Следовательно, сечение $\gamma_{\hat{s}}$ входит снизу в правый клюв фронта $\Sigma$. Отсюда $F_{L, \lambda}\left(a^{r}\right)<0$ по лемме 8.5. Утверждение леммы 8.3 доказано.

8.9. Сведение к случаю $\lambda$-общего узла. Докажем утверждение теоремы 7.3 для произвольных (необших) лежандровых узлов $L \in \mathscr{L}_{1}$. Малым шевелением мы превратим узел $L$ в $\lambda$-обший без увеличения числа точек множества $N_{\lambda}^{\mathrm{S}}(L)$ (мы предполагаем, что это множество конечно). Для гладкой функции $\eta \in C^{\infty}\left(S^{1}\right)$ обозначим через $\Psi_{\eta}$ контактоморфизм

$$
(p, q, u) \mapsto\left(p+\eta^{\prime}(q), q, u+\eta(q)\right) .
$$

Утверждение теоремы сводится к случаю $\lambda$-обшего узла с помощью двух следующих лемм.

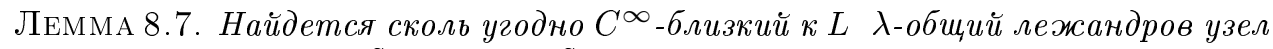
$L_{0} \in \mathscr{L}_{1}$ mакой, что $\# N_{\lambda}^{\mathrm{S}}\left(L_{0}\right) \leqslant \# N_{\lambda}^{\mathrm{S}}(L)$.

Лемма 8.8. Если $L_{0} \in \mathscr{L}_{1}-$ достаточно $C^{\infty}-$-лизкий $\kappa L \quad \lambda$-общий лежсандров узел, то найдется такая функиия $\eta \in C^{\infty}\left(S^{1}\right)$, что лежсандров узел $L_{1}=\Psi_{\eta}\left(L_{0}\right)$ удовлетворяет условиям: (1) \# $N_{\lambda}^{\mathrm{S}}\left(L_{1}\right)=\# N_{\lambda}^{\mathrm{S}}\left(L_{0}\right)$; (2) функиия $H_{L_{1}}$ ортогональна функииям $1, \sin q, \cos q, \ldots, \sin k q, \cos k q$.

ДокАЗАТЕльство лЕммы 8.7. Докажем сначала следующее утверждение.

Лемма 8.9. Пусть $F_{L, \lambda}(x)=0, U$ - такая окрестность точки $x$ в $J^{1}\left(S^{1}\right)$, что $x$ - единственный нуль отображения $F_{L, \lambda}$ на множестве $L \cap U$. Найдется сколь угодно $C^{\infty}-$-лизкий $\kappa L$ лежсандров узел $L_{U} \in \mathscr{L}_{1}$, совпадающий с $L$ вне $U$, такой, что выполнено следующее: (1) если $F_{L, \lambda}$ не меняет знак в точке $x$, то $F_{L_{U}, \lambda}$ не обращается в нуль на $L_{U} \cap U ;(2)$ если $F_{L, \lambda}$ меняет знак в точке $x$, то найдется ровно одна такая точка $x^{\prime} \in L_{U} \cap U$, что $F_{L_{U}, \lambda}\left(x^{\prime}\right)=0$, при этом $d F_{L_{U}, \lambda}\left(x^{\prime}\right) \neq 0 u \mu_{L_{U}}\left(x^{\prime}\right)=\mu_{L}(x)$.

ДокАЗАТЕЛЬСТВо. В малой окрестности точки $x=\left(p_{0}, q_{0}, u_{0}\right)$ узел $L$ совпадает с 1-графиком функции $f$, определенной на интервале $W \subset S^{1}$. Выберем функцию $g: W \rightarrow \mathbb{R}$ с компактньм носителем так: если функция $f^{\prime \prime}+\lambda f$ не меняет знак в точке $q_{0}$, то $g$ положительна и постоянна в окрестности точки $q_{0}$; если $f^{\prime \prime}+\lambda f$ меняет знак в точке $q_{0}$, то $g(q)$ совпадает с $q-q_{0}$ в окрестности точки $q_{0}$. Обозначим через $L_{t}$ лежандрово многообразие, полученное из $L$ заменой 1-графика функщии $f$ на 1-график функции $f+t g$. Для всех узлов одного из семейств $\left\{L_{\varepsilon}\right\}$ или $\left\{L_{-\varepsilon}\right\}$ при достаточно малых $\varepsilon>0$ вьполнено утверждение леммы (утверждение о совпадении значений масловских потенциалов следует из $C^{\infty}$-близости).

Применим лемму 8.9 ко всем точкам множества $N_{\lambda}^{S}(L)$, выбирая окрестности $U$ попарно не пересекаюшимися. У полученного узла $L^{\prime}$ все нули отображения $F_{L^{\prime}, \lambda}$ регулярны. Следовательно, сколь угодно $C^{\infty}$-малым шевелением узел $L^{\prime}$ можно сделать $\lambda$-обшим, не меняя числа точек множества $N_{\lambda}^{\mathrm{S}}\left(L^{\prime}\right)$. Доказательство леммы 8.7 закончено. 
ДоКАЗАТЕЛЬСТВо ЛЕммы 8.8. Пользуясь леммой 5.3, выберем отрезок $I \subset S^{1}$ таким, что множество $\sigma\left(L_{0}\right) \cap J^{0}(I)$ состоит из неособых точек фронта $\sigma\left(L_{0}\right)$ и его прообраз в $L_{0}$ не содержит точек множества $N_{\lambda}^{\mathrm{S}}\left(L^{\prime}\right)$.

Всякая непрерывная функция $\eta$ на $S^{1}$ определяет функционал $\eta^{*}$ на пространстве $\mathrm{T}^{k}$ тригонометрических многочленов степени не вьше $k$ при помощи $L^{2}$-спаривания: $\eta^{*}(f)=\int_{S^{1}} \eta(q) f(q) d q$. Следуюшее простое утверждение хорошо известно.

ЛЕмма 8.10. Найдутся такие функиии $\eta_{1}, \ldots, \eta_{2 k+1} \in C^{\infty}\left(S^{1}\right)$ с носителем в I, что функиионалы $\eta_{1}^{*}, \ldots, \eta_{2 k+1}^{*}$ линейно независимы.

Рассмотрим функционал $H_{L_{0}}^{*}$ на пространстве $\mathrm{T}^{k}$. Согласно лемме 8.10 , найдется ровно одна линейная комбинация $\eta$ функций $\eta_{1}^{*}, \ldots, \eta_{2 k+1}^{*}$ такая, что $\eta^{*}=-H_{L_{0}}^{*}$. Положим $L_{1}=\Psi_{\eta}\left(L_{0}\right)$. Из $C^{0}$-близости $L_{0}$ и $L$ следует, что функции $H_{L_{0}}$ и $H_{L}$ $C^{0}$-близки (согласно утверждению 7.1) и, значит, функция $\eta C^{\infty}$-мала. Отсюда вытекает, что узлы $L_{1}$ и $L C^{\infty}$-близки, если $L_{0}$ и $L$ достаточно $C^{\infty}$-близки. Поэтому $N_{\lambda}^{\mathrm{S}}\left(L_{1}\right)=N_{\lambda}^{\mathrm{S}}\left(L_{0}\right)$, так как $L_{1}$ и $L_{0} C^{\infty}$-близки к $L$ и могут различаться лишш на множестве $\{(p, q, u) \mid q \in I\}$.

Для завершения доказательства леммы 8.8 остается проверить, что $H_{L_{1}}^{*}=0$. Это вытекает из следующего утверждения.

Лемма 8.11. Для любого $L^{\prime} \in \mathscr{L}_{1}$ и любой функиии $\eta \in C^{\infty}\left(S^{1}\right)$ верно

$$
H_{\Psi_{\eta}\left(L^{\prime}\right)}=H_{L^{\prime}}+\eta
$$

ДокАЗАТЕЛЬСТво. Обозначим через $U$ всюду плотное подмножество $S^{1}$, состоящее из регулярных значений проекщии $L^{\prime} \rightarrow S^{1}$. Рассмотрим семейство узлов $L_{t}^{\prime}=$ $\Psi_{t \eta}\left(L^{\prime}\right), t \in[0,1]$. Непрерьвно зависящая от $t$ функция $H_{L_{t}^{\prime}}-t \eta$ в действительности не зависит от $t$, поскольку в каждой из точек множества $U$ она может принимать лишш конечное число значений. Поэтому $H_{\Psi_{\eta}\left(L^{\prime}\right)}-\eta=H_{L^{\prime}}$.

Доказательство теоремы 7.3 закончено.

8.10. Доказательство теоремы 7.4. Рассмотрим лежандров узел $L_{c}=\Psi_{c}(L) \in$ $\mathscr{L}_{1}$, полученньй из узла $L$ сдвигом на $c$ вдоль координаты $u$. Если $c=-\int_{S^{1}} H_{L}(q) d q$, то по лемме 8.11 функция $H_{L_{c}}$ ортогональна функциям $1, \cos q, \ldots, \sin k q$, т.е. узел $L_{c}$ удовлетворяет условию теоремы 7.3. При таком сдвиге $\lambda$-точки Арнольда узла $L$ переходят в $\lambda$-точки Арнольда узла $L_{c}$. Поэтому утверждение теоремы следует из теоремы 7.3 и следующей леммы, являющейся вариантом леммы Ролля.

ЛЕмма 8.12. У любого отрезка $J \subset L$ с концами в точках множества $N_{\lambda}^{\mathrm{S}}(L)$ есть по крайней мере одна внутренняя точка, принадлежащая множеству $N_{\lambda}^{\mathrm{A}}(L)$.

ДоказАТЕЛЬство. Рассмотрим гладкий путь $l:[0,1] \rightarrow L$, диффеоморфно параметризующий отрезок $J$. Из того, что его концы принадлежат множеству $N_{\lambda}^{\mathrm{S}}(L)$, получаем $m(l)=0$. По лемме 8.4 отсюда вытекает, что $\mathrm{i}_{\lambda}(l)=0$. Поэтому всякое поднятие $\widetilde{l}_{\lambda}:[0,1] \rightarrow \mathbb{R}$ отображения $F_{L, \lambda} \circ l:[0,1] \rightarrow \mathbb{R P}^{1}$ удовлетворяет условию $\widetilde{l}_{\lambda}(0)=\widetilde{l}_{\lambda}(1)$. По лемме Ролля найдется такое $\left.t \in\right] 0,1\left[\right.$, что $\widetilde{d l}_{\lambda}(t)=0$ и, следовательно, $d F_{L, \lambda}(l(t))=0$. Тем самым, $l(t)$ есть $\lambda$-точка Арнольда. 
8.11. Доказательство теоремы 7.5. Мы считаем, что число критических точек узла $L \subset \mathscr{L}_{1}$ конечно. Рассуждая как в доказательстве теоремы 7.3, мы можем свести утверждение теоремы 7.5 к случаю, когда лежандров узел $L$ является $\sigma$-общим и образы критических точек узла $L$ под действием проекции $\sigma$ являются неособыми точками фронта $\Sigma=\sigma(L)$.

Пусть функция $H_{L}: S^{1} \rightarrow \mathbb{R}$ и множество $Y=Y(L) \subset S^{1}$ определены как в доказательстве теоремы 7.3. Производная $H_{L}^{\prime}$ функции $H_{L}$ - определенная на $S^{1} \backslash Y$ гладкая функция, имеющая ненулевые односторонние пределы в точках множества $Y$. Функция $H_{L}^{\prime} L^{2}$-ортогональна тригонометрическим многочленам степени не вьше $k$. Тогда функция $H_{L}^{\prime}$ меняет знак не менее $2 k$ раз (перемены знака в точках множества $Y$ также учитьваются). Это следует из теоремы Гурвица (применимо доказательство леммы 8.1).

Если $H_{L}^{\prime}$ меняет знак в точке $s_{0} \in S^{1} \backslash Y$, то точка узла $L$, проектирующаяся в точку $\left(s_{0}, H_{L}\left(s_{0}\right)\right),-$ критическая. Согласно леммам 8.2 и 6.3 , всякая точка $s \in Y$ определяет отрезок $J_{\hat{s}}^{*}\left(D_{L}\right) \subset L$, где $\hat{s}=\left(s, H_{L}(s)\right) \in \Gamma_{H_{L}}$. Если функция $H_{L}^{\prime}$ меняет знак в точке $s \in Y$, то значения координаты $p$ в концах отрезка $J_{\hat{s}}^{*}\left(D_{L}\right)$ различны по знаку. Поэтому $J_{\hat{s}}^{*}\left(D_{L}\right)$ содержит хотя бы одну критическую точку узла $L$. Из леммы 6.3 следует, что все построенные критические точки различны.

\section{§. Гипотезы Арнольда}

9.1. Доказательство теоремы 0.1. Согласно лемме 1.1, точки касания лежандрова зацепления $L \in S T^{*} \mathbb{R}^{2}$ со слоями проекции $\rho$ - это в точности нули отображения $F_{L}$. Поскольку функция $F_{L}$ совпадает с функцией $F_{L, 1}$, определенной в п. 7.1 , то точки касания $L$ со слоями проекции $\rho$ - это в точности 1 -точки Штурма зацепления $L$ (нули отображения $F_{L, 1}$ ).

Мы можем считать, что начало координат на плоскости находится внутри фронтов $\rho\left(L_{0}\right)$ и $\rho\left(L_{1}\right)$. Используя отождествление $S T^{*} \mathbb{R}^{2}$ и $J^{1}\left(S^{1}\right)$ (см. рис. 3 ), получаем, что фронт $\sigma\left(L_{0}\right)$ - график отрицательной функции $f_{0} \in C^{\infty}\left(S^{1}\right)$, фронт $\sigma\left(L_{1}\right)$ - график положительной функции $f_{0} \in C^{\infty}\left(S^{1}\right)$. Согласно утверждению 7.1 , семейство лежандровых узлов $\left\{L_{t}\right\}$ определяет семейство $\left\{H_{t}=\mathscr{H}\left(L_{t}\right)\right\}$ непрерывных функций на $S^{1}$. Очевидно, $f_{0}=H_{0}, f_{1}=H_{1}$. Следовательно, найдется такое $t_{0} \in[0,1]$, что $\int_{S^{1}} H_{t_{0}}(q) d q=0$. Применяя к $L_{t_{0}}$ теорему 7.3 с $\lambda=1$ и $k=0$, получаем, что узел $L_{t_{0}}$ касается слоев проекции $\rho$ по меньшей мере в четырех точках. Основное утверждение теоремы 0.1 доказано.

Предположим теперь, что семейство $\left\{L_{t}\right\}$ общее в том смысле, что в семействе фронтов $\left\{\rho\left(L_{t}\right)\right\}$ встречаются лишь перестройки, изображенные на рис. $1 \mathrm{a}-\mathrm{e}$. Покажем, что среди фронтов $\left\{\rho\left(L_{t}\right)\right\}$ найдется фронт с по меньшей мере четырьмя невырожденными клювами. По уже доказанному найдется такой узел $L_{t_{0}}$, что проекция $\left.\rho\right|_{t_{0}}$ имеет не меньше четырех критических точек. Если $t_{0}-$ не момент перестройки, то утверждение доказано. Если $t_{0}$ - момент перестройки, то фронты $\left\{\rho\left(L_{t}\right)\right\}$ при $t$, принадлежащем по крайней мере одной из двух достаточно малых полуокрестностей точки $t_{0}$, имеют по меньшей мере 4 невырожденных клюва.

9.2. Доказательство теоремы 0.3. Вершины лежандрова зацепления $L$ - это в точности 1-точки Арнольда, определенные в п. 7.2. Применение теоремы 7.4 (с $\lambda=1$ и $k=0$ ) доказывает теорему 0.3 . 
9.3. Ручные узлы. Аналоги теорем 0.1 и 0.3 справедливы и для некоторых других компонент пространства лежандровых узлов в $S T^{*} \mathbb{R}^{2}=J^{1}\left(S^{1}\right)$.

По каждой связной компоненте $\mathscr{L}_{0}$ пространства лежандровых зацеплений в $J^{1}\left(S^{1}\right)$ можно построить пространство $\mathscr{L}_{0}^{+}$лежандровых зацеплений так же, как в $\S 7$ по компоненте $\mathscr{L}_{1}$ строилось пространство $\mathscr{L}_{1}^{+}$(зацепление $L^{+} \in \mathscr{L}_{0}^{+}$получается из зацепления $L \in \mathscr{L}_{0}$ добавлением компоненты $V_{c}=\{u=c, p=0\}$, фронт которой лежит вьше фронта $\sigma(L))$. Определена естественная проекщия $\mathscr{L}_{0}^{+} \rightarrow \mathscr{L}_{0}$.

Пусть $L^{+} \in \mathscr{L}_{0}^{+}-\sigma$-обшее зацепление. Назовем псевдоинволюцию $\mathrm{P}^{+}$фронта $\sigma\left(L^{+}\right)$ручной, если она положительна и $\chi\left(\mathrm{P}^{+}\right)=0$. Компонента $\mathscr{L}_{0}$ пространства лежандровых узлов называется ручной, если фронт какого-то (и тогда любого по теореме 2.5) $\sigma$-общего лежандрова зацепления из $\mathscr{L}_{0}^{+}$допускает ручную псевдоинволю-

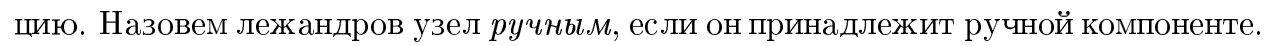

Лемма 9.1. Пусть $L \subset J^{1}\left(S^{1}\right)-$ ручной $\sigma$-общий узел. Тогда число Маслова $m(L)$ узла $L$ равно нулю. Предположим, что $\mathrm{P}^{+}-$ручная псевдоинволюиия фронта $\sigma\left(L^{+}\right)$, где $L^{+}=L \cup V_{c}-$ поднятие узла $L$ в $\mathscr{L}_{0}^{+}$. Тогда существует ровно один такой челочисленный масловский потенииал $\mu_{\mathrm{P}}+$ на $L^{+}$, равный 1 на $V_{c}$, что псевдоинволючия $\mathrm{P}^{+}$является масловской относительно $\mu_{\mathrm{P}}+$.

ДокАЗАтЕльство. Рассмотрим разбиение $D_{\mathrm{P}+}$ фронта $\sigma\left(L^{+}\right)$, порожденное псевдоинволюцией $\mathrm{P}^{+}$. Отбрасывая сечение $\sigma\left(V_{c}\right)$, получаем разбиение $D_{-}$фронта $\sigma(L)$. Рассуждая как в доказательстве леммы 8.2 , получаем, что разбиение $D_{-}$ручное в смысле определения, данного в п. 6.2. Докажем, что для любой точки $x \in \mathrm{Sw}\left(D_{-}\right)$индекс Маслова пути $l_{x}$, диффеоморфно параметризующего отрезок $J_{x}^{*}\left(D_{-}\right)$, определенньй в лемме 6.3, равен нулю. Действительно, по лемме 6.3 всякий клюв фронта $\sigma(L)$ является концом ровно одного сечения $\gamma_{x^{\prime}}$, где $x^{\prime} \in \mathrm{Sw}\left(D_{-}\right)$. Прообразы в $R(D)$ клювов, являюшихся конщами сечения $\gamma_{x^{\prime}}$, лежат в одной компоненте разрешения $R(D)$. Из леммы 6.2 следует, что прообразы этих клювов в $L$ либо оба содержатся, либо оба не содержатся в $J_{x}^{*}\left(D_{-}\right)$. Поскольку разбиение $D_{-}$получается из псевдоинволюции, сечение $\gamma_{x^{\prime}}$ либо входит в оба клюва сверху, либо в оба снизу. Поэтому при вычислении индекса Маслова пути $l_{x}$ эти клювы учитываются с противоположньми знаками. Следовательно, $m\left(l_{x}\right)=0$.

Обозначим $\Gamma=\mathrm{P}^{+}\left(\sigma\left(V_{c}\right)\right)$. Из леммы 6.3 вытекает, что число Маслова узла $L$ равно сумме по всем $x \in \mathrm{Sw}\left(D_{-}\right) \cap \Gamma$ индексов Маслова путей $l_{x}$, подсчитанных с некоторыми знаками. Значит, $m(L)=0$.

Пусть $y_{0} \in \Gamma$ - неособая точка фронта $\sigma(L), z_{0}$ - ее прообраз в $L, z_{1} \in V_{c}$ - такая точка, что $\mathrm{P}^{+}\left(y_{0}\right)=\sigma\left(z_{1}\right)$. Масловский потенщиал $\mu_{\mathrm{P}}+$ однозначно определяется тем, что он принимает значение 1 на $V_{c}$ и значение 0 в точке $z_{0}$. Для всякой переключающей точки $x$ вьполнено $m\left(l_{x}\right)=0$ и, следовательно, значения $\mu_{\mathrm{P}}+$ на ее прообразах в $L$ совпадают. Поскольку $\mu_{\mathrm{P}^{+}}\left(z_{1}\right)=\mu_{\mathrm{P}^{+}}\left(z_{0}\right)+1$, псевдоинволюция $\mathrm{P}^{+}$масловская относительно $\mu_{\mathrm{P}+}$ по утверждению 2.4. Отсюда, в свою очередь, следует, что $\mu_{\mathrm{P}+}$ обрашается в нуль на прообразах в $L$ неособых точек фронта $\sigma(L)$, принадлежаших $\Gamma$. Поэтому определение масловского потенциала $\mu_{\mathrm{P}+}$ не зависит от выбора точки $y_{0}$.

\section{4. Гипотезы Арнольда для ручных узлов.}

ТЕОрема 9.2. Пусть $\left\{L_{t \in[0,1]}\right\}$ - такой гладкий путь в ручной компоненте пространства лежандровых узлов, что ограничение координаты и на $L_{0}$ отри- 
цательно, а на $L_{1}$ положительно. Найдется такое $t_{0} \in[0,1]$, что лежсандров узел $L_{t_{0}}$ касается слоев проекичи $\rho$ по меньшей мере в четырех точках.

ТЕОРема 9.3. Всякий ручной лежандров узел имеет по меньшей мере четыре вериины.

9.5. Пример. Приведем пример ручного лежандрова узла с гладким $\rho$-фронтом, не принадлежашего изучавшейся ранее компоненте $\mathscr{L}_{1}$.

Опишем вначале удобньй геометрический способ построения по фронту $\sigma(L) \subset$ $J^{0}\left(S^{1}\right)$ фронта $\rho\left(L_{1}\right) \subset \mathbb{R}^{2}$ лежандрова узла $L_{1}$ из той же связной компоненты пространства $\mathscr{L}$, что и $L$. Фронт $\rho\left(L_{1}\right)$ есть образ фронта $\sigma(L)$ при вложении цилиндра $J^{0}\left(S^{1}\right)$ в $\mathbb{R}^{2}:(q, u) \mapsto\left(e^{u} \cos q, e^{u} \sin q\right)$, фронт $\rho\left(L_{1}\right)$ коориентирован от начала координат.

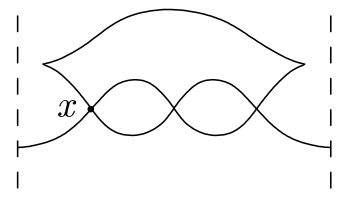

a

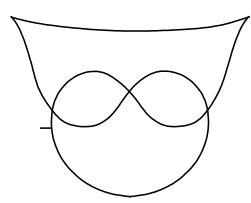

b

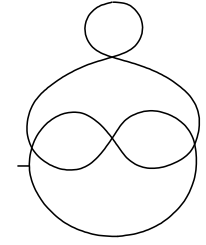

c

Рис. 13

При этом вложении $\sigma$-фронт узла $L^{\prime}$, изображенньй на рис. $13 \mathrm{a}$, переходит во фронт, диффеоморфный изображенному на рис. $13 \mathrm{~b}$. После этого несложно проверить, построив соответствующую последовательность перестроек фронтов на плоскости, что узел $L \subset S T^{*} \mathbb{R}^{2}$, фронт которого $\rho(L)$ приведен на рис. 13 с, принадлежит той же связной компоненте $\mathscr{L}^{\prime}$ пространства $\mathscr{L}$, что и узел $L^{\prime}$. Эта компонента ручная, поскольку $\sigma\left(L^{\prime}\right)$ допускает ручную псевдоинволюцию $\mathrm{P}$, определяемую условием $\mathrm{Sw}(\mathrm{P})=\{x\}$. Компоненты $\mathscr{L}^{\prime}$ и $\mathscr{L}_{1}$ различны, так как узел $L^{\prime}$ не изотопен в классе гладких вложений сечению расслоения $J^{1}\left(S^{1}\right) \rightarrow S^{1}$. По теореме 9.3 у всякого лежандрова узла из компоненты $\mathscr{L}^{\prime}$ есть по меньшей мере четыре вершины.

9.6. Теоремы Гурвица для ручных узлов. Ключевую роль в доказательстве теорем 9.2 и 9.3 играют теоремы 9.4 и 9.5 - аналоги теорем 7.3 и 7.4 для ручных компонент. Пусть $L-\sigma$-обший лежандров узел из ручной компоненты $\mathscr{L}_{0}$. Рассмотрим его поднятие $L^{+}=L \cup V_{c}$ в $\mathscr{L}_{0}^{+}$. По теореме 2.5 фронт $\sigma\left(L^{+}\right)$допускает хотя бы одну ручную псевдоинволюцию Р. Обозначим через $H_{L, \mathrm{P}}: S^{1} \rightarrow \mathbb{R}$ непрерьвную функцию, график которой совпадает с $\mathrm{P}\left(\sigma\left(V_{c}\right)\right) \in \sigma(L)$, через $\mathscr{R}(L)$ - множество таких функций $H: S^{1} \rightarrow \mathbb{R}$, что $H=H_{L, \mathrm{P}}$ для какой-нибудь ручной псевдоинволющии Р. Множество $\mathscr{R}(L)$ всегда конечно. Число элементов в $\mathscr{R}(L)$, вообще говоря, различно для узлов $L$, принадлежаших разным компонентам пространства $\mathscr{L}_{0} \backslash \mathscr{D}$.

Определим для каждого $L \in \mathscr{L}_{0} \cap \mathscr{D}$ множество $\mathscr{R}(L)$ непрерывных функций на $S^{1}$, задающих непрерьвные сечения фронта $\sigma(L)$ : функция $H$ принадлежит множеству $\mathscr{R}(L)$, если и только если найдутся последовательность $\left(L_{i}\right)$ узлов из $\mathscr{L}_{0} \backslash \mathscr{D}, C^{\infty}$-сходяшаяся к $L$, и последовательность функций $H_{i} \in \mathscr{R}\left(L_{i}\right), C^{0}$-сходяшаяся к $H$. Рас- 
суждая как в доказательстве теоремы 5.1 , можно показать, что множество $\mathscr{R}(L)$ непусто и конечно.

Из лемме 7.2 вытекает, что на всяком ручном лежандровом узле есть целочисленньй масловский потенциал, однозначно определенньй с точностью до прибавления константы. Следующие теоремы являются обобщениями теорем 7.3 и 7.4.

Tеорема 9.4. Пусть $L-$ такой ручной узел, ито найдется функиия $H \in \mathscr{R}(L)$, ортогональная $2 k+1$ функииям $1, \cos q, \sin q, \ldots, \cos k q, \sin k q$. Тогда для любого $\lambda \in \mathbb{R}$ на $L$ найдется по меньшей мере $2 k+2 \lambda$-точки Штурма, в которых значения иелочисленного масловского потенциала совпадают. Если $\lambda=(k+1)^{2}$, то на $L$ найдется по меньшей мере $2 k+4 \lambda$-точки Штурма, в которых значения челочисленного масловского потенииала совпадают.

ТеОрема 9.5. Пусть $L-$ такой ручной узел, что найдется функиия $H \in \mathscr{R}(L)$, ортогональная $2 k$ функциям $\cos q, \sin q, \ldots, \cos k q, \sin k q$. Тогда для любого $\lambda \in \mathbb{R}$ справедливьг неравенства \# $\left(N_{\lambda}^{\mathrm{A}}(L)\right) \geqslant 2 k+2, \#\left(N_{(k+1)^{2}}^{\mathrm{A}}(L)\right) \geqslant 2 k+4$.

Доказательства этих теорем практически дословно повторяют доказательства теорем 7.3 и 7.4. Единственное отличие состоит в том, что при доказательстве утверждения теоремы 9.4 для необщих узлов вместо утверждения 7.1 используется следующее свойство непрерывности: для любого $L \in \mathscr{L}_{0}$ и любой функции $H \in \mathscr{R}(L)$ верно, что если узел $L^{\prime}$ достаточно $C^{\infty}$-близок к $L$, то $\mathscr{R}\left(L^{\prime}\right)$ содержит функцию, достаточно $C^{0}$-близкую к $H$.

9.7. Двойственные проективные пространства и лежандровы проекции. Мы переходим к обсуждению некоторых вопросов В.И. Арнольда о фронтах на проективной плоскости.

Для любого многообразия $N$ контактное многообразие $S T^{*} N$ является естественным двулистным накрытием над контактным многообразием $P T^{*} N$ некоориентированных контактных элементов в $N$. Естественная проекция $\rho^{\times}: P T^{*} N \rightarrow N$ является лежандровой. Пусть $N=\mathbb{R} \mathrm{P}^{n}$. Рассмотрим проективное пространство $\left(\mathbb{R} \mathrm{P}^{n}\right)^{\vee}$, двойственное к пространству $\mathbb{R} \mathrm{P}^{n},-$ многообразие гиперплоскостей $((n-1)$-мерных проективных подпространств) пространства $\mathbb{R P}^{n}$. Помимо проекции $\rho^{\times}: P T^{*} \mathbb{R} P^{n} \rightarrow$ $\mathbb{R} \mathrm{P}^{n}$, определена естественная проекция $\rho^{\vee}: P T^{*} \mathbb{R} \mathrm{P}^{n} \rightarrow\left(\mathbb{R} \mathrm{P}^{n}\right)^{\vee}$. Проекция $\rho^{\vee}$ переводит контактньй элемент в единственную гиперплоскость, которой он касается.

9.8. Клювы и перегибы фронтов на проективной плоскости. Ограничимся рассмотрением случая $n=2$. Легко проверить, что если $x$ - точка обшего лежандрова зацепления $L \subset P T^{*} \mathbb{R} P^{2}$, то фронт $\rho^{\times}(L)$ имеет клюв в точке $\rho^{\times}(x)$ тогда и только тогда, когда фронт $\rho^{\vee}(L)$ имеет перегиб в точке $\rho^{\vee}(x)$. И наоборот $-\not$ фронт $\rho^{\times}(L)$ имеет перегиб в точке $\rho^{\times}(x)$ тогда и только тогда, когда фронт $\rho^{\vee}(L)$ имеет клюв в точке $\rho^{\vee}(x)$.

9.9. Некоориентированные фронты на плоскости. Сформулируем гипотезу, родственную гипотезе Арнольда о трех перегибах из [3]: если $\left\{L_{t}\right\}-$ общий путь в пространстве лежандровых узлов в $P T^{*} \mathbb{R} P^{2}$ такой, что $L_{0}-$ слой проекции $\rho^{\times}$, то фронт $\rho^{\times}\left(L_{1}\right)$ имеет по меньшей мере три клюва (и, следовательно, фронт $\rho^{\vee}\left(L_{1}\right)$ имеет по меньшей мере три перегиба). Верна ли эта гипотеза - неизвестно. Мы можем 
доказать лиш более слабое утверждение, о фронтах лежандровых многообразий в $P T^{*} \mathbb{R}^{2} \subset P T^{*} \mathbb{R} P^{2}$.

Рассмотрим связную компоненту $\mathscr{L}_{1}^{\times}$пространства лежандровых узлов в $P T^{*} \mathbb{R}^{2}$, содержашую слой естественной проекции $\rho^{\times}: P T^{*} \mathbb{R}^{2} \rightarrow \mathbb{R}^{2}$.

Теорема 9.6. Всякий лежандров узел $L \in \mathscr{L}_{1}^{\times}$касается слоев проекции $\rho^{\times}$ по крайней мере в трех точках. Для общего узла $L \in \mathscr{L}_{1}^{\times}$фронт $\rho^{\times}(L)$ имеет по меньшей мере три клюва.

ДокАЗАТЕльство. Обозначим через $\overline{\mathscr{L}}$ пространство лежандровых зацеплений в $J^{1}\left(S^{1}\right)$, инвариантных относительно действия контактоморфизма $\Delta: J^{1}\left(S^{1}\right) \rightarrow$ $J^{1}\left(S^{1}\right),(p, q, u) \mapsto(-p, q+\pi,-u)$. Прообразы в $S T^{*} \mathbb{R}^{2}$ лежандровых зацеплений в $P T^{*} \mathbb{R}^{2}$ - это в точности лежандровы зацепления из $\overline{\mathscr{L}}$ (мы используем отождествление $S T^{*} \mathbb{R}^{2}$ и $J^{1}\left(S^{1}\right)$ ). Обозначим через $\overline{\mathscr{L}}_{1}$ множество лежандровых узлов из $\mathscr{L}_{1}$, инвариантных относительно $\Delta$. Поскольку компонента $\mathscr{L}_{1}$ содержит лежандров узел $V_{0}=\{u=p=0\}$, являюшийся поднятием в $J^{1}\left(S^{1}\right)$ слоя проекции $\rho^{\times}$над началом координат, теорема 9.6 вытекает из следующей теоремы.

Теорема 9.7. На всяком лежандровом узле из $\overline{\mathscr{L}}_{1}$ найдется по меньшей мере шесть 1-точек Штурма.

ДоказАТельство. Пусть $L \in \overline{\mathscr{L}}_{1}$. Рассмотрим функцию $H_{L}=\mathscr{H}(L)$, определенную в утверждении 7.1 .

Лемма 9.8. Если $L \in \overline{\mathscr{L}}_{1}$, то функция $H_{L}$ нечетная в том смысле, что $H_{L}(q+\pi)=-H_{L}(q)$ для любого $q \in S^{1}=\mathbb{R} / 2 \pi \mathbb{Z}$.

Теорема 9.7 следует из леммы 9.8 и теоремы 7.3. Действительно, из леммы 9.8 вытекает, что функция $H_{L}$ ортогональна функциям $1, \cos 2 q, \sin 2 q$. Пусть $\eta(q)=a_{1} \sin q+$ $a_{2} \cos q$. Лежандров узел $L_{\eta}=\Psi_{\eta}(L)$ принадлежит пространству $\overline{\mathscr{L}}_{1}$. Согласно лемме 8.11, $H_{L_{\eta}}=H_{L}+\eta$ и можно выбрать числа $a_{1}, a_{2}$ так, что функция $H_{L_{\eta}}$ будет ортогональна функциям $1, \cos q, \sin q, \cos 2 q, \sin 2 q$. Согласно теореме 7.3, узел $L_{\eta}$ содержит по меньшей мере шесть 1-точек Штурма. Поскольку контактоморфизм $\Psi_{\eta}$ взаимно однозначно отображает 1 -точки Штурма узла $L$ в 1 -точки Штурма узла $L_{\eta}$, узел $L$ также содержит по меньшей мере шесть 1-точек Штурма.

ДоКАЗАТЕЛЬСТво ЛЕммы 9.8. Утверждение леммы достаточно доказать для обших узлов, на необшие узлы оно распространяется по непрерьвности с помошью утверждения 7.1. Пусть $L$ - обший лежандров узел, инвариантньй относительно инволюции $\Delta$. Возьмем такое $c>0$, что фронт $\sigma\left(V_{c}\right)$ лежит вьше фронта $\sigma(L)$, а фронт $\sigma\left(V_{-c}\right)$ лежит ниже фронта $\sigma(L)$. Положим $L_{+}=L \cup V_{c}, L_{-}=L \cup V_{-c}$. Обозначим через $B\left(L_{ \pm}\right)$множество положительных псевдоинволюций фронта $\sigma\left(L_{ \pm}\right)$. Взаимно однозначное отображение $T: B\left(L_{+}\right) \rightarrow B\left(L_{-}\right)$определим следующим условием: $T(\mathrm{P})(x)=\mathrm{P}(x)$, если $x \notin \sigma\left(V_{c}\right)$ и $\mathrm{P}(x) \notin \sigma\left(V_{c}\right)$. Пусть $\Delta^{\prime}: J^{0}\left(S^{1}\right) \rightarrow J^{0}\left(S^{1}\right),(q, u) \mapsto$ $(q+\pi,-u)$. Зададим взаимно однозначное отображение $T_{\Delta}: B\left(L_{+}\right) \rightarrow B\left(L_{-}\right)$формулой $T_{\Delta}(\mathrm{P})(x)=\Delta^{\prime} \mathrm{P} \Delta^{\prime}(x)$. Согласно утверждению 2.6 , множество $B\left(L_{+}\right)$содержит ровно одну псевдоинволюцию, обозначим ее Р. Утверждение леммы сводится к тому, 
что сечение $\mathrm{P}\left(\sigma\left(V_{c}\right)\right) \subset \sigma(L)$ инвариантно относительно действия инволюции $\Delta^{\prime}$. Эта инвариантность вытекает из равенства

$$
\Delta^{\prime}\left(\mathrm{P}\left(\sigma\left(V_{c}\right)\right)=\left(T^{-1} T_{\Delta}(\mathrm{P})\right)\left(\sigma\left(V_{c}\right)\right)=\mathrm{P}\left(\sigma\left(V_{c}\right)\right) .\right.
$$

Доказательство леммы 9.8 и теоремы 9.7 закончено.

9.10. Неустранимость клювов. Если $L$-лежандров узел в $S T^{*} \mathbb{R}^{2}$ с $m(L)=0$, то его можно продеформировать в классе лежандровых иммерсий в лежандров узел, $\rho$-фронт которого является гладкой иммерсированной кривой. Если запретить самопересечения, то это, вообше говоря, неверно: для любого $k$ найдется такая компонента $\mathscr{L}^{k}$ пространства лежандровых узлов, что индекс Маслова любого лежандрова узла из $\mathscr{L}^{k}$ равен нулю и $\rho$-фронт всякого общего лежандрова узла из $\mathscr{L}^{k}$ имеет по меньшей мере $2 k$ клювов [14], [15]. Это утверждение несложно также доказать, используя методы настояшей работы.

\section{$\S$ 10. Критические точки лежандровых зацеплений}

Критической точкой лежсандрова подмногообразия $L \subset J^{1}(M)$ называется такая точка $(p, q, u) \in L$, что $p=0$. Это определение обобщает определение критической точки функции на $M$. Пусть $M=S^{n}$. Точка $z \in L$ является критической тогда и только тогда, когда (при отождествлении $J^{1}\left(S^{n}\right)$ и $S T^{*} \mathbb{R}^{n+1}$ ) вектор $\rho(z) \in \mathbb{R}^{n+1}$ ортогонален гиперплоскости, касательной к фронту $\rho(L)$ в точке $\rho(z)$. Таким образом, критическим точкам $L$ соответствуют нормали к фронту $\rho(L)$, проходяшие через начало координат.

10.1. Положительные зацепления и их критические точки. Пусть $M=$ $S^{1}$. Назовем $\sigma$-общее лежандрово зацепление $L \subset J^{1}\left(S^{1}\right)$ положительнылм, если фронт зацепления $L^{+}=L \cup V_{c}$ (такого, что $\sigma\left(V_{c}\right)$ лежит выше $\sigma(L)$ ) допускает положительную псевдоинволюцию. Компонента $\mathscr{L}_{0}$ пространства лежандровых зацеплений называется положительной, если какое-нибудь (и тогда любое по теореме 2.5) $\sigma$-обшее лежандрово зацепление из $\mathscr{L}_{0}^{+}$положительно. Назовем лежандрово зацепление положительным, если оно принадлежит положительной компоненте. Определим для положительного лежандрова зацепления $L$ множество $\mathscr{R}^{\prime}(L)$ непрерывных функций на $S^{1}$ так же, как в п. 9.6 определялось множество $\mathscr{R}(L)$ для древесного узла $L$; единственное отличие состоит в том, что в определении участвуют все (а не только ручные) положительные псевдоинволюции фронта $\sigma\left(L \cup V_{c}\right)$. Множество $\mathscr{R}^{\prime}(L)$ непусто и конечно. Следующая теорема является обобщением теоремы 7.5.

ТеОрема 10.1. Пусть $L$ - такое положительное лежандрово зачепление, что найдется функция $H \in \mathscr{R}^{\prime}(L)$, ортогональная $2 k$ функциям $\cos q, \sin q, \ldots$, $\cos k q, \sin k q$. Тогда на $L$ найдется по меньшей мере $2 k+2$ критические точки.

В частности, любое положительное лежандрово зацепление имеет по меньшей мере две критические точки. Отметим, что можно привести пример положительного зацепления, состоящего из двух связных компонент, для каждой из которых найдется не содержаший критических точек лежандров узел, лежащий в той же компоненте пространства лежандровых узлов, что и она. 
Критическая точка $z$ лежандрова зацепления $L \subset J^{1}\left(S^{1}\right)$ назьвается невырожденной, если $\sigma(z)$ является неособой точкой фронта $\sigma(L)$ и ограничение координаты $p$ на $L$ имеет невырожденный нуль в точке $z$. Назовем лежандрово зацепление $L \subset J^{1}\left(S^{1}\right)$ (и его фронт $\left.\sigma(L)\right)$ С-общим, если зацепление $L$ является $\sigma$-обшим и все его критические точки невырождены. В доказательстве теоремы 10.1 ключевую роль играет следуюшее утверждение.

УТВЕРЖДЕНИЕ 10.2. Пусть L - С-общее положительное лежандрово зацепление, $H \in \mathscr{R}^{\prime}(L)$. Тогда число критических точек зачепления $L$ не меньше числа локальных әкстремумов функиии $H$.

Прежде чем перейти к доказательству утверждения 10.2 и теоремы 10.1, дадим необходимые определения и докажем некоторые вспомогательные утверждения.

10.2. Внешние и внутренние критические точки. Назовем невырожденную критическую точку $z \in L$ точкой максимума (минимума), если ограничение координаты $u$ на $L$ имеет в точке $z$ локальньй максимум (минимум). Пусть $\mathrm{P}-$ псевдоинволюция С-обшего фронта $\sigma(L)$. Назовем критическую точку $z \mathrm{P}$-внешней, если либо $z$ - точка максимума и точка $\mathrm{P}(\sigma(z))$ лежит ниже точки $\sigma(z)$, либо $z$ - точка минимума и точка $\mathrm{P}(\sigma(z))$ лежит вьше точки $\sigma(z)$. Назовем критическую точку $\mathrm{P}$-внутренней, если она не является Р-внешней точкой. Обозначим через $B_{L}$ число критических точек зацепления $L$, через $B_{L, \mathrm{P}}^{\mathrm{E}}\left(\right.$ соответственно $\left.B_{L, \mathrm{P}}^{\mathrm{I}}\right)$ число Р-внешних (соответственно Р-внутренних) критических точек зацепления $L$.

10.3. Сглаживание. Пусть $X$ - подмножество множества точек самопересечения С-обшего фронта $\sigma(L)$ лежандрова зацепления $L$. Назовем сәлаживанием зацепления $L$ относительно множества $X$ (неоднозначно определенное) лежандрово зацепление $L^{\prime}$, фронт которого получается следующей конструкцией. Зафиксируем для каждой точки $x \in X$ ее малую окрестность $U_{x} \subset J^{0}\left(S^{1}\right)$. Фронт $\sigma\left(L^{\prime}\right)$ лежандрова зацепления $L^{\prime}$ совпадает с $\sigma(L)$ вне объединения $U$ окрестностей $U_{x}$. Пересечение фронта $\sigma\left(L^{\prime}\right)$ с каждой из окрестностей $U_{x}$ состоит из двух гладких непересекающихся ветвей. Выберем эти гладкие ветви так, что либо на каждом из их прообразов в $L^{\prime}$ найдется по одной критической точке (если $p$-координаты прообразов в $L$ точки $x$ имеют разные знаки), либо на их прообразах в $L^{\prime}$ нет критических точек (если $p$-координаты прообразов в $L$ точки $x$ имеют один знак). Если $\mathrm{P}$ - положительная псевдоинволюция фронта $\sigma(L)$ и $X \subset \mathrm{Sw}(\mathrm{P})$, то сушествует ровно одна такая положительная псевдоинволюция $\mathrm{P}^{\prime}$ фронта $\sigma\left(L^{\prime}\right)$, что для любой неособой точки $y$ фронта $\sigma\left(L^{\prime}\right)$, удовлетворяющей условиям $y \notin U, \mathrm{P}(y) \notin U$, вьполнено $\mathrm{P}^{\prime}(y)=\mathrm{P}(y)$. Назовем псевдоинволюцию $\mathrm{P}^{\prime}$ сглаживанием псевдоинволюиии $\mathrm{P}$ относительно множества $X$.

Лемма 10.3. Пусть Р - положительная псевдоинволюиия б-общего фрон$m a \sigma(L), X \subset \mathrm{Sw}(\mathrm{P})$. Тогда

$$
B_{L^{\prime}, \mathrm{P}^{\prime}}^{\mathrm{E}}-B_{L^{\prime}, \mathrm{P}^{\prime}}^{\mathrm{I}} \geqslant B_{L, \mathrm{P}}^{\mathrm{E}}-B_{L, \mathrm{P}}^{\mathrm{I}}
$$

где $L^{\prime}, \mathrm{P}^{\prime}$ получаются из $L, \mathrm{P}$ сглаживанием относительно множества $X$. 
ДокАЗАТЕльСТво. Множество критических точек лежандрова зацеплении $L^{\prime}$, не принадлежащих прообразу никакой из окрестностей $U_{x} \subset J^{0}\left(S^{1}\right)$, где $x \in X$, совпадает с множеством критических точек $L^{\prime}$. При этом Р-внутренние критические точки являются $\mathrm{P}^{\prime}$-внутренними, $\mathrm{P}$-внешние $-\mathrm{P}^{\prime}$-внешними. Прообраз в $L^{\prime}$ всякой окрестности $U_{x}$ либо не содержит критических точек лежандрова зацепления $L^{\prime}$, либо содержит ровно две критические точки. В последнем случае из положительности псевдоинволюции $\mathrm{P}$ следует, что либо они обе $\mathrm{P}^{\prime}$-внешние, либо одна $\mathrm{P}^{\prime}$-внешняя, а другая $\mathrm{P}^{\prime}$-внутренняя.

Лемма 10.4. Пусть $L_{0} \subset J^{1}\left(S^{1}\right)$ - С-общее лежандрово зачепление, $\mathrm{P}_{0}-$ положительная псевдоинволюиия фронта $\sigma\left(L_{0}\right)$. Тогда число $\mathrm{P}_{0}$-внутренних критических точек не меньше числа $\mathrm{P}_{0}$-внешних критических точек.

ДоказАтельство. Предположим, что множество $\mathrm{Sw}\left(\mathrm{P}_{0}\right)$ пусто. Тогда число $\mathrm{P}_{0^{-}}$ внутренних критических точек равно числу $\mathrm{P}_{0}$-внешних критических точек. Это следует из того, что на каждой из связных компонент зацепления $L_{0} \mathrm{P}_{0}$-внутренние и $\mathrm{P}_{0}$-внешние критические точки чередуются.

Предположим теперь, что множество $\mathrm{Sw}\left(\mathrm{P}_{0}\right)$ непусто. Рассмотрим сглаживание $L_{0}^{\prime}$ лежандрова зацепления $L_{0}$ относительно $\mathrm{Sw}\left(\mathrm{P}_{0}\right)$. Обозначим через $\mathrm{P}_{0}^{\prime}$ положительную псевдоинволюцию фронта $\sigma\left(L_{0}^{\prime}\right)$, получающуюся при сглаживании из $\mathrm{P}_{0}^{\prime}$. Тогда $\mathrm{Sw}\left(\mathrm{P}_{0}^{\prime}\right)$ пусто и $0=B_{L_{0}^{\prime}, \mathrm{P}_{0}^{\prime}}^{\mathrm{E}}-B_{L_{0}^{\prime}, \mathrm{P}_{0}^{\prime}}^{\mathrm{I}} \geqslant B_{L_{0}, \mathrm{P}_{0}}^{\mathrm{E}}-B_{L_{0}, \mathrm{P}_{0}}^{\mathrm{I}}$ по лемме 10.3.

10.4. Доказательство утверждения 10.2. Обозначим через $X$ подмножество графика $\Gamma \subset J^{0}\left(S^{1}\right)$ функции $H$, состоящее из точек излома графика. Рассмотрим сглаживание $L^{\prime}$ лежандрова зацепления $L$ относительно $X$. Пусть $\mathrm{P}$ - такая положительная псевдоинволюция фронта $\sigma\left(L \cup V_{c}\right)$ (где $\sigma\left(V_{c}\right)$ лежит выше $\sigma(L)$ ), что $\Gamma=\sigma\left(V_{c}\right)$. Тогда $X \subset \mathrm{Sw}(P)$. Обозначим через $\mathrm{P}^{\prime}$ сглаживание псевдоинволюции Р относительно $X$. Рассмотрим лежандрово зацепление $L^{\prime \prime}$, фронт которого есть $\sigma\left(L^{\prime}\right) \backslash \mathrm{P}^{\prime}\left(V_{c}\right)$. Ограничивая псевдоинволюцию $\mathrm{P}^{\prime}$, получаем положительную псевдоинволюцию фронта $\sigma\left(L^{\prime \prime}\right)$, обозначим ее $\mathrm{P}^{\prime \prime}$.

Обозначим через $W$ множество критических точек $L^{\prime \prime}$, являюшихся критическими точками $L$, через $B_{H}$ - число критических точек $L$, проектируюшихся в график $\Gamma$, через $b$ - число точек, в которых функция $H$ негладкая и имеет локальный экстремум. Мы должны доказать, что $B_{L} \geqslant B_{H}+b$. Из каждого негладкого локального экстремума функции $H$ при сглаживании появляется одна критическая точка зацепления $L^{\prime \prime}$; все критические точки $L^{\prime \prime}$, не принадлежашие $W$, возникают таким образом. Из положительности псевдоинволюции Р вытекает, что все критические точки $L^{\prime \prime}$, не принадлежашие $W$, являются $\mathrm{P}^{\prime \prime}$-внешними. Поэтому $\#(W) \geqslant B_{L^{\prime \prime}, \mathrm{P}^{\prime \prime}}^{\mathrm{I}}$ и $B_{L^{\prime \prime}, \mathrm{P}^{\prime \prime}}^{\mathrm{E}} \geqslant b$. Из этих неравенств и неравенства $B_{L^{\prime \prime}, \mathrm{P}^{\prime \prime}}^{\mathrm{I}} \geqslant B_{L^{\prime \prime}, \mathrm{P}^{\prime \prime}}^{\mathrm{E}}$, доставляемого леммой 10.4 , вытекает $\#(W) \geqslant b$. Поскольку $B_{L}=B_{H}+\#(W)$, получаем $B_{L} \geqslant B_{H}+b$.

10.5. Замечание об индексах Маслова критических точек. Пусть $L-$ С-обший ручной лежандров узел, $\mathrm{P}$ - ручная псевдоинволюция фронта $\sigma\left(L \cup V_{c}\right)$, $\mu_{\mathrm{P}}$ - определяемый псевдоинволюцией $\mathrm{P}$ масловский потенциал на $L$ (см. лемму 9.1), $H \in \mathscr{R}(L)$ - непрерьвная функция на $S^{1}$, график которой есть $\mathrm{P}\left(\sigma\left(V_{c}\right)\right)$. Обозначим через $\nu_{i}(L)$ число критических точек зацепления $L$, значение масловского потенциала $\mu_{\mathrm{P}}$ в которых равно $i$. Можно показать, что справедливо следующее усиление утверж- 
дения 10.2: если функция $H$ имеет $2 k$ локальных экстремумов, то $\nu_{-1}(L)+\nu_{0}(L) \geqslant k$, $\nu_{1}(L)+\nu_{0}(L) \geqslant k, \nu_{-1}(L)+\nu_{0}(L)+\nu_{1}(L) \geqslant 2 k$.

10.6. Доказательство теоремы 10.1. Рассуждая как в доказательстве теоремы 7.3, можно показать (мы опускаем подробности), что утверждение достаточно проверить для С-общего зацепления $L$. Функция $\frac{d}{d q} H$ - гладкая функция на $S^{1} \backslash Y$, где $Y \subset S^{1}$ - конечное множество. В каждой из точек множества $Y$ производная $H^{\prime}$ функции $H$ имеет ненулевые левые и правые пределы. Функщия $H^{\prime} L^{2}$-ортогональна тригонометрическим многочленам степени не выше $k$. Функция $H^{\prime}$ меняет знак не меньше $2 k+2$ раз (перемены знака в точках множества $Y$ также учитьваются). Это следует из теоремы Гурвица (применимо доказательство леммы 8.1). То есть функция $H$ имеет по меньшей мере $2 k+2$ локальных экстремума. Теорема теперь следует из утверждения 10.2 .

\section{§11. Инварианты лежандровых зацеплений}

11.1. Классические инварианты. Теория псевдоинволюций позволяет определить инварианты лежандровых зацеплений в $J^{1}(M)$ (инварианты - это локально постоянные функции на пространстве лежандровых зацеплений $\mathscr{L}$ ). Для простоты ограничимся описанием инвариантов лежандровых узлов в $J^{1}(\mathbb{R})$. Напомним определение классических инвариантов (неориентированного) лежандрова узла. Это, во-первых, гладкий тип узла, во-вторых, число Маслова $m(L)$, определенное в $\S 2$, и, в-третьих, число Терстона-Беннекена $\beta(L)$. Число Терстона-Беннекена определяется следуюшим образом. Зафиксируем ориентацию на $L$ (результат не будет зависеть от ее выбора). Коэффищиент зацепления узла $L$ и узла $L^{\prime}$, полученного из $L$ небольшим сдвигом вдоль координаты $u$, назьвается числом Терстона-Беннекена $\beta(L)$. Для общего лежандрова узла число $\beta(L)$ получается подсчетом числа правых клювов и самопересечений со знаками:

$$
\beta(L)=\#(\not)+\#(X)-\#(X)-\#(\not)-\#(\searrow)
$$

Число Маслова и число Терстона-Беннекена - единственные инварианты конечного порядка (в смысле Васильева), не сводяшиеся к инвариантам конечного порядка гладких узлов [17].

11.2. Псевдоинволюции и инварианты. Обозначим через $\mu_{L}$ единственньй (с точностью до константы) масловский потенциал на лежандровом узле $L \in J^{1}(\mathbb{R})$, принимающий значения в $\mathbb{Z} / m(L) \mathbb{Z}$. Для $\sigma$-общего лежандрова узла $L$ обозначим через $\mathrm{PI}(L)$ число масловских относительно $\mu_{L}$ положительных псевдоинволюций фронта $\sigma(L)$, через $\mathrm{PI}^{\prime}(L)$ число всех положительных псевдоинволюций фронта $\sigma(L)$. Согласно утверждению 2.6 , каждая из функций PI, PI' принимает одно и то же значение на всех $\sigma$-общих лежандровых узлах из одной связной компоненты пространства лежандровых узлов. Поэтому, распространяя функции PI и PI' на необщие узлы, получаем инварианты лежандровых узлов.

11.3. Пример. Рассмотрим лежандровы узлы $L$ и $L^{\prime}$, фронты которых $\Sigma=\sigma(L)$ и $\Sigma^{\prime}=\sigma\left(L^{\prime}\right)$ изображены на рис. 14 . Их классические инварианты совпадают: $m(L)=$ $m\left(L^{\prime}\right)=0, \beta(L)=\beta\left(L^{\prime}\right)=1$, оба узла имеют гладкий тип $5_{2}$. Покажем, что они 
различаются инвариантом РІ. Следовательно, содержащие их компоненты пространства $\mathscr{L}$ различны. То, что эти компоненты не совпадают, было ранее показано другим способом в [11] и независимо Я. М. Элиашбергом (не опубликовано).

$\Phi$ ронт $\Sigma$ допускает ровно две положительные псевдоинволющии. Эти псевдоинволюции $\mathrm{P}_{0}, \mathrm{P}_{1}$ задаются условиями $\mathrm{Sw}\left(\mathrm{P}_{0}\right)=\left\{x_{1}, x_{2}, x_{5}, x_{6}\right\}$ и $\mathrm{Sw}\left(\mathrm{P}_{1}\right)=\left\{x_{1}, x_{2}, x_{3}\right.$, $\left.x_{4}, x_{5}, x_{6}\right\}$. Фронт $\Sigma^{\prime}$ также допускает ровно две положительные псевдоинволюции. Эти псевдоинволюции $\mathrm{P}_{0}^{\prime}, \mathrm{P}_{1}^{\prime}$ задаются условиями $\mathrm{Sw}\left(\mathrm{P}_{0}^{\prime}\right)=\left\{y_{1}, y_{4}, y_{5}\right\}$ и $\mathrm{Sw}\left(\mathrm{P}_{1}^{\prime}\right)=$ $\left\{y_{1}, y_{2}, y_{3}, y_{4}, y_{5}\right\}$. Таким образом, $\mathrm{PI}^{\prime}(L)=\mathrm{PI}^{\prime}\left(L^{\prime}\right)=2$. Все точки самопересечения фронта $\Sigma$ масловские, а из точек самопересечения фронта $\Sigma^{\prime}$ масловскими (относительно целочисленного масловского потенциала) являются лишь $y_{1}, y_{4}, y_{5}$. Поэтому псевдоинволюции $\mathrm{P}_{0}, \mathrm{P}_{1}, \mathrm{P}_{0}^{\prime}$ масловские, а $\mathrm{P}_{1}^{\prime}$ нет. Значит, $\operatorname{PI}(L)=2, \operatorname{PI}\left(L^{\prime}\right)=1$.
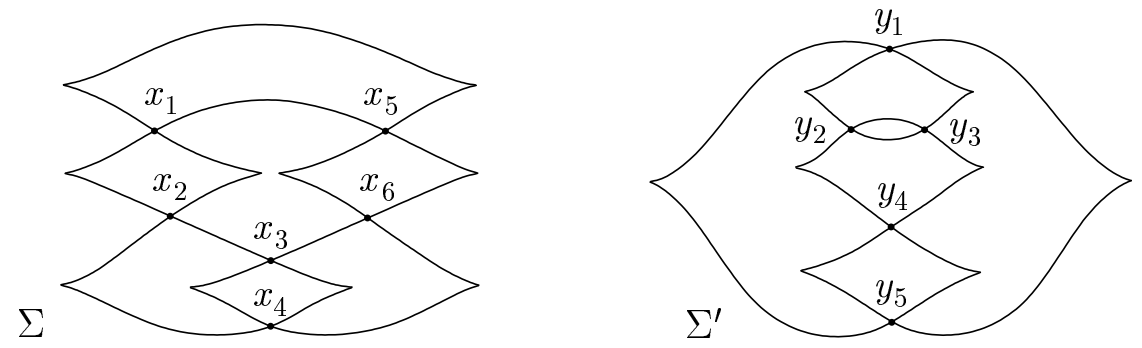

Рис. 14

Заметим, что примеры, в которых инвариант $\mathrm{PI}^{\prime}$ различал бы лежандровы узлы с совпадающими классическими инвариантами, неизвестны.

11.4. Уточнение инвариантов. В действительности, теория псевдоинволюций позволяет определить и более тонкие инварианты, родственные инвариантам PI, PI' Пусть $m$ и $k$-неотрицательные целые числа. Для общего лежандрова узла $L$ определим число $\mathrm{PI}_{m, k}(L)$ следуюшим образом. Если $m$ - делитель числа $m(L)$, то $\mathrm{PI}_{m, k}(L)$ есть число таких положительных псевдоинволюций $\mathrm{P}$ фронта $\sigma(L)$, масловских относительно масловского потенциала $\mu_{L} \otimes \mathbb{Z} / m \mathbb{Z}$ (принимающего значение в $\mathbb{Z} / m \mathbb{Z}$ ), что $\chi(\mathrm{P})=1-k$. В случае, когда $m=1$, это определение говорит, что $\mathrm{PI}_{1, k}(L)$ есть число таких положительных псевдоинволюций $\mathrm{P}$ фронта $\sigma(L)$, что $\chi(\mathrm{P})=1-k$. Наконец, полагаем $\mathrm{PI}_{m, k}(L)=0$, если $m$ не делит $m(L)$.

Согласно утверждению 2.6 , каждая из функций $\mathrm{PI}_{m, k}$ является инвариантом лежандровых узлов. Определим многочлены Пуанкаре $\mathrm{PI}_{m}^{L}$ следуюшим образом:

$$
\mathrm{PI}_{m}^{L}(t)=\sum_{k} \mathrm{PI}_{m, k}(L) t^{k}
$$

Заметим, что

$$
\mathrm{PI}(L)=\mathrm{PI}_{m(L)}^{L}(1), \quad \mathrm{PI}^{\prime}(L)=\mathrm{PI}_{1}^{L}(1) .
$$

Если $L \in J^{1}(\mathbb{R})-\sigma$-общий лежандров узел, то всякая $\sigma$-общая петля в $\mathscr{L}$ с началом в $L$ определяет в силу утверждения 2.6 взаимно однозначное отображение множества 
положительных псевдоинволющий фронта $\sigma(L)$ в себя. Эти отображения образуют группу монодромии положительных псевдоинволюций фронта $\sigma(L)$. В $\S 4$ было показано, что эта группа может быть нетривиальна. Пользуясь действием групшы монодромии, можно определить дополнительные инварианты лежандровых узлов (например, вычисляя число орбит действия). Отметим, что множество положительных псевдоинволюций, подсчитанных числом $\mathrm{PI}_{m, k}(L)$, инвариантно относительно действия группы монодромии.

\section{5. Эйлерова характеристика и число Терстона-Беннекена.}

Лемма 11.1. Пусть $\mathrm{P}$ - положительная псевдоинволюиия фронта $\sigma(L) \sigma$-общего лежандрова узла $L \in J^{1}(\mathbb{R})$. Тогда $\chi(\mathrm{P}) \equiv \beta(L)(\bmod 2)$.

Таким образом, $\mathrm{PI}_{m, k}(L)=0$, если число $k+\beta(L)$ четно.

ДокАЗАТЕльство ЛЕммы 11.1. Число Терстона-Беннекена $\beta(L)$ сравнимо по модулю 2 с суммой числа правых клювов и числа точек самопересечений фронта $\Sigma=$ $\sigma(L)$. Эйлерова характеристика $\chi(\mathrm{P})$ равна разности числа правых клювов и числа переключаюших точек псевдоинволюции Р. Поэтому достаточно показать, что число непереключающих точек псевдоинволюции Р четно. Обозначим через $D$ разбиение фронта $\Sigma$, определяемое псевдоинволюцией Р. Компоненты пространства разрешения $R(D)$ (см. п. 6.1) гомеоморфны окружностям. Пусть $S, S^{\prime}$ - две различные компоненты. Рассмотрим их образы $\psi_{D}(S), \psi_{D}\left(S^{\prime}\right)$ под действием проекции $\psi_{D}: R(D) \rightarrow \Sigma$. Докажем, что число точек пересечения $\psi_{D}(S)$ и $\psi_{D}\left(S^{\prime}\right)$, являюшихся непереключающими для $\mathrm{P}$, четно. Это следует из того, что индекс пересечения по модулю 2 циклов $\psi_{D}(S)$ и $\psi_{D}\left(S^{\prime}\right)$ равен нулю, каждая непереключающая точка из $\psi_{D}(S) \cap \psi_{D}\left(S^{\prime}\right)$ дает вклад 1 в индекс пересечения, а каждая переключающая точка из $\psi_{D}(S) \cap \psi_{D}\left(S^{\prime}\right)$ дает вклад 0 . Поскольку всякая непереключающая точка самопересечения фронта $\Sigma$ принадлежит образам ровно двух компонент пространства $R(D)$, общее число непереключающих точек четно, и утверждение леммы доказано.

11.6. Связная сумма и инварианты $\mathrm{PI}_{m, k}$. Пусть ориентированные лежандровы узлы $L_{1}, L_{2}$ в $J^{1}(\mathbb{R})$ таковы, что их фронты $\Sigma_{1}=\sigma\left(L_{1}\right), \Sigma_{2}=\sigma\left(L_{2}\right)$ вьглядят как показано на рис. 15 . Связной суммой узлов $L_{1}$ и $L_{2}$ называется узел $L, \phi р$ ронт которого изображен на рис. 15 справа. Можно показать, что операция связной суммы задает однозначную операцию над связньми компонентами пространства ориентированных лежандровых узлов, т.е. в соответствующих компонентах можно выбрать узлы требуемого вида и связная компонента, в которой лежит получающийся узел, не зависит от этого выбора.
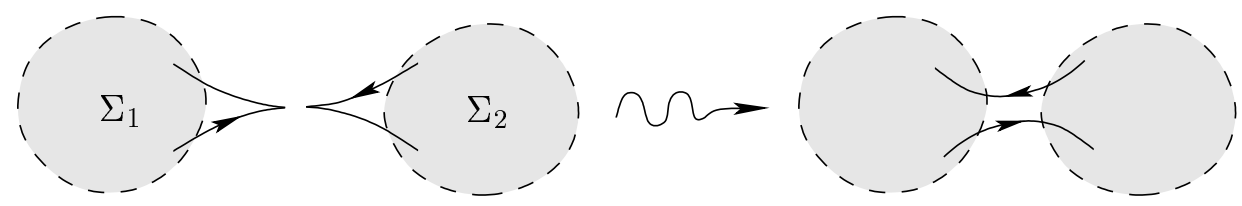

Рис. 15 
УТВЕРЖДЕНИЕ 11.2. Пусть лежандров узел L есть связная сумма лежандровихх узлов $L_{1}, L_{2} \in J^{1}(\mathbb{R})$. Тогда $\mathrm{PI}_{1}^{L}(t)=\mathrm{PI}_{1}^{L_{1}}(t) \cdot \mathrm{PI}_{1}^{L_{2}}(t), \mathrm{PI}_{2}^{L}(t)=\mathrm{PI}_{2}^{L_{1}}(t) \cdot \mathrm{PI}_{2}^{L_{2}}(t)$ и если $m\left(L_{1}\right)=m\left(L_{2}\right)=0($ и тогда $m(L)=0), m o \mathrm{PI}_{0}^{L}(t)=\mathrm{PI}_{0}^{L_{1}}(t) \cdot \mathrm{PI}_{0}^{L_{2}}(t)$.

ДокАЗАТЕЛЬСТво. Если $\mathrm{P}_{1}$ - положительная псевдоинволюция фронта $\sigma\left(L_{1}\right)$, $\mathrm{P}_{2}$ - положительная псевдоинволюция фронта $\sigma\left(L_{2}\right)$, то существует ровно одна положительная псевдоинволюция $\mathrm{P}$ фронта $\sigma(L)$ с $\mathrm{Sw}(\mathrm{P})=\operatorname{Sw}\left(\mathrm{P}_{1}\right) \cup \mathrm{Sw}\left(\mathrm{P}_{2}\right)$. Более того, всякая положительная псевдоинволюция фронта $\sigma(L)$ получается такой конструкцией. Отсюда следует, что $\mathrm{PI}_{1}^{L}(t)=\mathrm{PI}_{1}^{L_{1}}(t) \cdot \mathrm{PI}_{1}^{L_{2}}(t)$. Пусть $x$ - точка самопересечения фронта $\sigma(L)$. Тогда ее прообразы в $L$ можно соединить в $L$ путем, которьй целиком лежит в $L_{1}$ или в $L_{2}$. Значит, при $m\left(L_{1}\right)=m\left(L_{2}\right)=0$ точка $x$ масловская для $L$ тогда и только тогда, когда $x$ масловская для $L_{1} \cup L_{2}$. Утверждение о масловости точки $x$ для масловских потенщиалов со значениями в $\mathbb{Z} / 2 \mathbb{Z}$ справедливо всегда, так как числа Маслова лежандровых узлов четны. Это завершает доказательство.

11.7. Уточнение теоремы о продолжении для лежандровых зацеплений. В более сложных случаях (когда зацепление состоит из нескольких компонент или база $M$ есть окружность) для построения дополнительных инвариантов может быть использована следующая конструкция фактора по псевдоинволюции.

Пусть $\mathrm{P}$ - псевдоинволюция $\sigma$-общего фронта $\Sigma \subset J^{0}(M)$, где $M=I, M=\mathbb{R}$ или $M=S^{1}$. На пространстве разрешения $R\left(D_{\mathrm{P}}\right)$ определена естественная непрерьвная инволюция $i_{\mathrm{P}}$, которая переставляет прообразы точек $x$ и $\mathrm{P}(x)$ при $\{x, \mathrm{P}(x)\} \subset \Sigma \backslash X_{\Sigma}$. Рассмотрим факторпространство $R\left(D_{\mathrm{P}}\right) / i_{\mathrm{P}}$; для $y \in R\left(D_{\mathrm{P}}\right)$ обозначим через $y / i_{\mathrm{P}}$ образ $y$ в $R\left(D_{\mathrm{P}}\right) / i_{\mathrm{P}}$. "Факторпространство" $\Sigma / \mathrm{P}$ получается из $R(\mathrm{P}) / i_{\mathrm{P}}$ склеиванием для каждой переключающей точки $x \in \mathrm{Sw}(\mathrm{P})$ двух таких точек $y / i_{\mathrm{P}}$ и $y^{\prime} / i_{\mathrm{P}}$, что $\psi_{D_{\mathrm{P}}}(y)=\psi_{D_{\mathrm{P}}}\left(y^{\prime}\right)=x$. Проекция $\Sigma \rightarrow M$ определяет естественное непрерьвное отображение $\mathrm{H}_{\mathrm{P}}: \Sigma / \mathrm{P} \rightarrow M$. Пространство $\Sigma / \mathrm{P}$ есть одномерньй клеточньй комплекс с вершинами двух типов: одновалентные вершины получаются из клювов фронта $\Sigma$ (и краевых точек при $M=I$ ), четырехвалентные - из переключаюших точек псевдоинволюции $\mathrm{P}$.

Пусть $\mathrm{P}_{i}$ - псевдоинволющия $\sigma$-общего фронта $\Sigma_{i}, i \in\{1,2\}$. Назовем псевдоинволюции $\mathrm{P}_{1}$ и $\mathrm{P}_{2}$ гомотопически подобными если существует такая гомотопическая эквивалентность $h: \Sigma_{1} / \mathrm{P}_{1} \rightarrow \Sigma_{2} / \mathrm{P}_{2}$, что отображения $\mathrm{H}_{\mathrm{P}_{2}} \circ h$ и $\mathrm{H}_{\mathrm{P}_{1}}$ гомотопически эквивалентны.

Пользуясь явным продолжением псевдоинволюции, описанным в доказательстве теоремы 2.5, можно показать, что верно следуюшее утверждение.

УТВеРЖДЕНИЕ 11.3. В условиях теоремы 2.5 отображсение $\mathrm{P}_{a} \mapsto \mathrm{P}_{b}$ переводит псевдоинволюцию в гомотопически подобную ей.

Для связных лежандровых зацеплений в $J^{1}(I)$ или $J^{1}(\mathbb{R})$ гомотопическое подобие псевдоинволюций эквивалентно совпадению их эйлеровых характеристик. В этой ситуации утверждение 11.3 не дает новой информации. А в других случаях, пользуясь этим утверждением, несложно построить дополнительные инварианты лежандровых зацеплений. 


\section{§12. Производящие семейства и псевдоинволюции}

В этом параграфе обсуждается связь теории производяших семейств лежандровых многообразий и теории псевдоинволюций.

12.1. Производящие семейства. Напомним вкратце конструкцию производящего семейства лежандрова многообразия (подробности см. в [5]). Пусть $M$ и $W-$ гладкие многообразия, $F: M \times W \rightarrow \mathbb{R}$ - гладкая функция. Для каждой точки $q \in M$ рассмотрим множество $B_{q} \subset\{q\} \times W$, которое состоит из критических точек ограничения функции $F$ на $\{q\} \times W$. Обозначим $B_{F}=\bigcup_{q \in M} B_{q} \subset M \times W$. Предположим, что ранг матрицы из вторых производных $\left(F_{w q}, F_{w w}\right)$ максимален (равен размерности многообразия $M$ ) в каждой точке множества $B_{F}$; для функции $F$ общего положения это условие вьполнено. Тогда множество $B_{F} \subset M \times W$ является гладким подмногообразием той же размерности, что и $M$, ограничение на $B_{F}$ отображения $(q, w) \stackrel{l_{F}}{\longmapsto}\left(q, d_{M}(F(q, w)), F(q, w)\right)\left(d_{M}\right.$ - первьй дифференциал вдоль $\left.M\right)$ задает лежандрову иммерсию многообразия $B_{F}$ в $J^{1}(M)$. В этом случае функция $F$ называется производящим семейством (иммерсированного) лежандрова многообразия $L_{F}=l_{F}\left(B_{F}\right)$.

Мы опишем некоторьй специальньй класс $\mathscr{G}$ производящих семейств и покажем, что всякая общая функция $F \subset \mathscr{G}$ определяет некоторую комбинаторную структуру на фронте $\sigma\left(L_{F}\right)$. Эта структура в случае $\operatorname{dim} M=1$ эквивалентна заданию положительной масловской псевдоинволюции на фронте.

Будем предполагать для простоты, что многообразие $M$ замкнуто. Пусть многообразие $W$ имеет вид $W=W_{0} \times \mathbb{R}^{N}$, где $W_{0}$ замкнуто, $N \geqslant 1$. Класс функций $\mathscr{G}$ состоит из производящих семейств, представимых в виде суммы ненулевой линейной функции на $\mathbb{R}^{N}$ и функции на $W$ с компактным носителем. Назовем такие семейства линейными на бесконечности. Также будем назьвать линейными на бесконечности функции на $W=W_{0} \times \mathbb{R}^{N}$, представимые в виде суммы ненулевой линейной функции на $\mathbb{R}^{N}$ и функции с компактным носителем.

Пусть $F \in \mathscr{G}$, точка $q_{0} \in M$ такова, что $F\left(q_{0}, \cdot\right): W \rightarrow \mathbb{R}$ - строго морсовская функция, т.е. такая морсовская функция, что все ее критические значения различны. Это условие вьполнено для точки обшего положения. Рассмотрим фронт $\Sigma_{F}=\sigma\left(L_{F}\right)$ лежандрова многообразия $L_{F}$ - образ $L_{F}$ под действием проекции $\sigma: J^{1}(M) \rightarrow J^{0}(M)$, $(p, q, u) \mapsto(q, u)$. Он состоит из таких точек $\left(q_{0}, u_{0}\right)$, что $u_{0}-$ критическое значение функции $F\left(q_{0}, \cdot\right): W \rightarrow \mathbb{R}$.

12.2. Комплексы Морса. Любая строго морсовская линейная на бесконечности функция $f$ на $W=W_{0} \times \mathbb{R}^{N}$ определяет разбиение множества своих критических значений на пары (это разбиение зависит также, как от параметра, от выбора поля коэффициентов, участвуюшего в конструкции). Построение этого разбиения на пары использует комплекс Морса, к описанию которого мы сейчас переходим. Зафиксируем на $W=W_{0} \times \mathbb{R}^{N}$ метрику $g_{0}$, являющуюся произведением евклидовой метрики на $\mathbb{R}^{N}$ и некоторой метрики на $W_{0}$. Пусть $g$-метрика на $W$, представимая в виде $g=g_{0}+h$, где $h$-тензорная 2-форма с компактным носителем на $W$. Обозначим через $\mathscr{M}_{g}\left(x^{\prime}, x^{\prime \prime}\right)$ множество траекторий $\gamma: \mathbb{R} \rightarrow M$ антиградиентного векторного поля $-\nabla_{g} f$, стремяшихся к $x^{\prime}$ при $t \rightarrow-\infty$ и к $x^{\prime \prime}$ при $t \rightarrow+\infty$. Обозначим $\widehat{\mathscr{M}}_{g}\left(x^{\prime}, x^{\prime \prime}\right)=\mathscr{M}_{g}\left(x^{\prime}, x^{\prime \prime}\right) / \mathbb{R}$, где $\mathbb{R}$ действует сдвигом параметра. 
Предположим, что метрика $g$ такова, что траектории множества $\widehat{\mathscr{M}}_{g}\left(x^{\prime}, x^{\prime \prime}\right)$, где $\operatorname{ind}\left(x^{\prime}\right)=\operatorname{ind}\left(x^{\prime \prime}\right)+1$ (ind - это индекс Морса критической точки), устойчивы относительно малых возмущений метрики $g$. Это условие вьполнено для метрик общего положения. Пусть $x_{1}, \ldots, x_{K}$ - критические точки функции $f$, занумерованные в порядке возрастания критических значений. Рассмотрим свободный градуированный $\mathbb{Z}$-модуль $C_{f}=\mathbb{Z} \otimes\left\{x_{1}, \ldots, x_{K}\right\}$, порожденньй образующими $x_{i}$, степень образующей равна ее индексу Морса. Определим оператор $\partial_{g}: C_{f} \rightarrow C_{f}$, задавая его действие на образующих формулой

$$
\partial_{g}\left(x_{k}\right)=\sum_{\operatorname{ind}\left(x_{j}\right)=\operatorname{ind}\left(x_{k}\right)-1} \#\left(\widehat{\mathscr{M}}_{g}\left(x_{k}, x_{j}\right)\right) x_{j}
$$

где траектории множества $\widehat{\mathscr{M}}_{g}\left(x_{k}, x_{j}\right)$ считаются с учетом должньм образом определенных знаков (строго говоря, для однозначного определения этих знаков надо фиксировать некоторые ориентации; произвол в этом месте приводит к преобразованию $\partial_{g}$ при помоши автоморфизма $\mathbb{Z}$-модуля $C_{f}$, меняюшего знаки некоторых образуюших). Отметим, что $\partial_{g}\left(x_{j}\right)$ есть линейная комбинация образуюших $x_{1}, \ldots, x_{j-1}$.

Хорошо известно (см., например, [22]), что $\partial_{g}^{2}=0$. Гомологии ker $\partial_{g} / \mathrm{im} \partial_{g}$ нулевые, так как они совпадают с целочисленнымигомологиями пары $(\{f \leqslant c\},\{f \leqslant-c\})$ топологических пространств, где $c$ - достаточно большое положительное число.

Рассмотрим групу $\operatorname{Aut}_{T}\left(C_{f}\right)$ верхнетреугольных автоморфизмов $\mathbb{Z}$-модуля $C_{f}$. Она состоит из градуированных автоморфизмов, которые сохраняют каждьй из подмодулей $\mathbb{Z} \otimes\left\{x_{1}, \ldots, x_{j}\right\} \subset C_{f}, j \in\{1, \ldots, K\}$. Можно показать, что различные дифференциалы $\partial_{g_{1}}$ и $\partial_{g_{2}}$, соответствующие разным выборам допустимых метрик $g_{1}$ и $g_{2}$, связаны соотношением

$$
\partial_{g_{2}}=A \partial_{g_{1}} A^{-1}
$$

где $A$ - некоторый элемент $\operatorname{Aut}_{\mathrm{T}}\left(C_{f}\right)$ (зависяший от $g_{1}$ и $\left.g_{2}\right)$.

12.3. $M$-дифференциалы и комбинаторика образующих $M$-комплекса. Аксиоматизируя свойства комплекса (дифференциального градуированного модуля) $\left(C_{f}, \partial_{g}\right)$, дадим следуюшее алгебраическое определение. Пусть $\mathbb{E}$ - коммутативное кольцо, $C$ - свободньй градуированньй $\mathbb{E}$-модуль с выделенной системой образующих $\left(x_{1}, \ldots, x_{K}\right)$. Назовем $M$-дифференииалом такое $\mathbb{E}$-линейное отображение $\partial: C \rightarrow C$ степени -1, что $\partial^{2}=0$ и $\partial\left(x_{j}\right) \in \mathbb{E} \otimes\left\{x_{1}, \ldots, x_{j-1}\right\}, j \in\{1, \ldots, K\} ;$

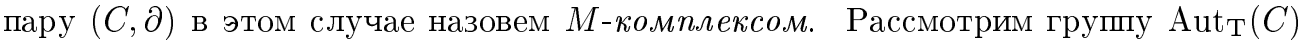
верхнетреугольных автоморфизмов $\mathbb{E}$-модуля $C$, состояшую из всех автоморфизмов, переводящих в себя каждый из подмодулей $\mathbb{E} \otimes\left\{x_{1}, \ldots, x_{j}\right\}, j \in\{1, \ldots, K\}$. Назовем два $M$-дифференциала $\partial_{1}, \partial_{2}: C \rightarrow C$ әквивалентными, если найдется такое $A \in \operatorname{Aut}_{\mathrm{T}}(C)$, что $\partial_{2}=A \partial_{1} A^{-1}$. Примером $M$-комплекса является пара $\left(C_{f} \otimes \mathbb{E}, \partial_{g} \otimes \mathbb{E}\right) ; M$-дифференциалы $\partial_{g_{1}} \otimes \mathbb{E}$ и $\partial_{g_{2}} \otimes \mathbb{E}$, построенные по различным метрикам $g_{1}$ и $g_{2}$, эквивалентны.

Назовем $M$-дифференциал $\partial$ әлементарным, если вьполнены следуюшие два условия: (1) для всякого $j \in\{1, \ldots, K\}$ либо $\partial\left(x_{j}\right)=0$, либо найдется такое $m \in\{1, \ldots, K\}$, что $\partial\left(x_{j}\right)=x_{m} ;(2)$ если $\partial\left(x_{j}\right)=\partial\left(x_{j^{\prime}}\right)=x_{m}$, то $j=j^{\prime}$. 
Теорема 12.1 [8], [6]. Пусть $(C, \partial)-M$-комплекс над полем $\mathbb{E}$. Тогда $M$-дифференииал д әквивалентен ровно одному из элементарных $М$-дифференииалов на $C$.

Элементарный $M$-дифференциал $\partial$ задает следующую комбинаторную структуру на множестве образующих: часть их представляет базис в гомологиях комплекса $(C, \partial)$, а остальные разбиты на пары $\left(x_{i}, x_{j}\right)$, где $\partial\left(x_{i}\right)=x_{j}$. В случае, когда $\mathbb{E}$ - поле, по теореме 12.1 произвольньй $M$-дифференциал определяет такую же структуру на множестве образуюших, и эти структуры одинаковы для эквивалентных $M$-дифференщиалов.

Отметим, что утверждение теоремы 12.1 неверно для случая $\mathbb{E}=\mathbb{Z}$ (уже в части сушествования). Тензорно домножая $M$-комплекс на различные поля $\mathbb{E}$, мы получаем, вообще говоря, разные комбинаторные структуры на множестве образующих. Можно показать, что если эти структуры совпадают для всех полей $\mathbb{Z} / p \mathbb{Z}$, то утверждение теоремы верно и для $\mathbb{E}=\mathbb{Z}$.

Пусть $f$ - строго морсовская линейная на бесконечности функция. Зафиксируем поле $\mathbb{E}$ раз и навсегда. По теореме 12.1 критические точки (и, следовательно, критически значения) функции $f$ канонически (т.е. независимо от выбора допустимой метрики $g$ ) разбиты на пары, так как гомологии комплекса $\left(C_{f} \otimes \mathbb{E}, \partial_{g} \otimes \mathbb{E}\right)$ равны нулю.

Можно показать, что сформулированное вьше дифференциально-алгебраическое определение разбиения на пары эквивалентно следуюшему топологическому определению. Два критических значения $a>b$ функции $f$ образуют пару, если и только если для любого достаточно малого $\varepsilon>0$ вьполнено условие

$$
\begin{aligned}
\operatorname{dim} H_{*}(\{f \leqslant a+\varepsilon\},\{f \leqslant b+\varepsilon\}) & =\operatorname{dim} H_{*}(\{f \leqslant a-\varepsilon\},\{f \leqslant b-\varepsilon\}) \\
& =\operatorname{dim} H_{*}(\{f \leqslant a-\varepsilon\},\{f \leqslant b+\varepsilon\})+1 \\
& =\operatorname{dim} H_{*}(\{f \leqslant a+\varepsilon\},\{f \leqslant b-\varepsilon\})+1 .
\end{aligned}
$$

12.4. От производящего семейства к псевдоинволюции. Пусть вложенное лежандрово многообразие $L_{F}$ задается производяшим семейством $F \in \mathscr{G}$. Пусть точка $q_{0} \in M$ такова, что прямая $\left\{q=q_{0}\right\} \subset J^{0}(M)$ пересекает фронт $\Sigma_{F}=\sigma\left(L_{F}\right)$ лишь в его неособых точках. Тогда функция $F\left(q_{0}, \cdot\right)$ строго морсовская. Тем самым на точках множества $\Sigma^{q_{0}}=\pi^{-1}\left(q_{0}\right) \cap \Sigma_{F}$ возникает свободная инволюция $i_{F}^{q_{0}}$. Из топологического определения разбиения на пары следует, что отображение $i_{F}^{q_{0}}$ непрерьвно зависит от точки $q_{0}$ и, более того, совокупность отображений $i_{F}^{q_{0}}$ продолжается до непрерьвного отображения $\mathrm{P}_{F}^{G}: G_{\Sigma_{F}} \rightarrow \Sigma_{F}$, где $G_{\Sigma_{F}}-$ множество неособых точек фронта $\Sigma_{F}$.

Предположим теперь, что $M=S^{1}$. Тогда непрерьвное отображение $\mathrm{P}_{F}^{G}$, определенное разбиением на пары, продолжается до псевдоинволюции $\mathrm{P}_{F}$ фронта $\Sigma_{F}$. Действительно, достаточно проверить, что ветви, входяшие в клюв, переставляются отображением $\mathrm{P}_{F}^{G}$, а ветви, входящие в точку трансверсального самопересечения, не переставляются. Рассмотрим неособые точки $\left(q, u_{1}\right),\left(q, u_{2}\right)$ фронта $\Sigma_{F}$, близкие к особой точке $\left(q_{0}, u_{0}\right) \in \Sigma_{F}$. Пусть $u_{1}>u_{2}$. Из теории Морса вытекает, что число $\operatorname{dim} H_{*}\left(\left\{F\left(q_{1}, \cdot\right) \leqslant u_{1}+\varepsilon\right\},\left\{F\left(q_{1}, \cdot\right) \leqslant u_{2}-\varepsilon\right\}\right)$ равно нулю, если $\left(q_{0}, u_{0}\right)-$ клюв, и равно двум, если $\left(q_{0}, u_{0}\right)$ - точка самопересечения. После этого требуемое утверждение легко вьводится из приведенного вьше топологического определения пары критических значений и теории Морса. 
На $L_{F}$ естественно возникает масловский потенциал $\mu_{F}: G_{L_{F}} \rightarrow \mathbb{Z}$, значение которого в точке $x=\left(p_{0}, q_{0}, u_{0}\right) \in G_{L_{F}}$ равно индексу Морса соответствующей критической точки функции $F\left(q_{0}, \cdot\right)$ с критическим значением $u_{0}$. Псевдоинволюция $\mathrm{P}_{F}$ масловская относительно $\mu_{F}$. Это следует из того, что если два критических значения $a>b$ функции $F\left(q_{0}, \cdot\right)$ образуют пару, то индекс Морса критической точки с критическим значением $a$ на единицу больше индекса критической точки с критическим значением $b$. Псевдоинволюция $\mathrm{P}_{F}$ положительна. Этот факт можно проверить, изучив перестройки разбиения критических значений на пары для обших однопараметрических семейств функций на многообразии $W$ (см. [6]). Само определение положительной (и масловской) псевдоинволюции является аксиоматизацией комбинаторных структур, возникаюших на фронте лежандрова многообразия, заданного производяшим семейством.

Можно также показать, что для всякой положительной псевдоинволюции $\mathrm{P}$ фронта $\sigma(L) \sigma$-общего зацепления $L$, масловской относительно некоторого целочисленного масловского потенциала $\mu$, найдется такое производящее семейство $F \in \mathscr{G}$, что $L=L_{F}, \mathrm{P}=\mathrm{P}_{F}$ и $\mu$ отличается от $\mu_{F}$ на константу (П.Е. Пушкарь, не опубликовано).

12.5. Сравнение семейств псевдоинволюций. Пусть $F: M \times W_{0} \times \mathbb{R}^{N} \rightarrow \mathbb{R}$ - производящее семейство лежандрова многообразия в $J^{1}(M)$. Назовем его стабилизацией производящее семейство

$$
\widetilde{F}: M \times W_{0} \times \mathbb{R}^{N} \times \mathbb{R}^{k} \rightarrow \mathbb{R}, \quad \widetilde{F}(x, y)=F(x)+Q(y),
$$

где $x \in M \times W_{0} \times \mathbb{R}^{N}, y \in \mathbb{R}^{k}, Q$ - невырожденная квадратичная форма на $\mathbb{R}^{k}$. Назовем два производящих семейства из $\widetilde{G}$ эквивалентньми, если некоторые их стабилизации, определенные на одном и том же многообразии $M \times W_{0} \times \mathbb{R}^{N}$, переводятся друг в друга расслоенным над $M$ диффеоморфизмом $M \times W_{0} \times \mathbb{R}^{N}$. Несложно проверить, что при $M=S^{1}$ псевдоинволющии, порождаемые эквивалентными производяшими семействами, совпадают.

Пусть $\left\{L_{t \in[0,1]}\right\}$ - гладкое семейство лежандровых многообразий в $J^{1}(M)$ и многообразие $L_{0}$ задается производяшим семейством $F \in \mathscr{G}$. Тогда, рассуждая как в [10], [13], [21], [9], можно показать, что найдется такое семейство $\left\{F_{t \in[0,1]}\right\}$ производящих семейств из $\mathscr{G}$, что $L_{F_{t}}=L_{t}$ и $F_{0}$ эквивалентно $F$. Интересно отметить, что для случая $\operatorname{dim} M=1$ доказательство сушествования такого семейства неявно содержится уже в [18]. Рассуждая как в [25], [24], можно показать, что если $\left\{\widetilde{F}_{t}\right\}-$ другое семейство, удовлетворяющее тем же требованиям, что и $\left\{F_{t}\right\}$, то при любом $t \in[0,1]$ производящее семейство $\widetilde{F}_{t}$ эквивалентно производящему семейству $F_{t}$.

Пусть $M=S^{1}$ и семейство зацеплений $L_{t} \sigma$-общее. Тогда семейство $\left\{F_{t}\right\}$ порождает непрерывное семейство $\left\{\mathrm{P}_{F_{t}}\right\}$ положительных псевдоинволюций фронтов $\sigma\left(L_{t}\right)$. Если $\left\{\widetilde{F}_{t}\right\}$ - другое семейство, удовлетворяющее тем же требованиям, что и $\left\{F_{t}\right\}$, то порождаемое им семейство псевдоинволюций совпадает с $\left\{\mathrm{P}_{F_{t}}\right\}$. Тем самым теория производящих семейств определяет каноническоепродолжение псевдоинволюции $\mathrm{P}_{F}$. Это наблюдение было отправной точкой для формулировки теоремы 2.5 .

Теорема 2.5 определяет продолжение псевдоинволюции $\mathrm{P}_{0}=\mathrm{P}_{F_{0}}$ до семейства $\left\{\mathrm{P}_{t \in[0,1]}\right\}$ псевдоинволюций фронтов $\sigma\left(L_{t}\right)$. Сравним семейства псевдоинволюций 
$\left\{\mathrm{P}_{t}\right\}$ и $\left\{\mathrm{P}_{F_{t}}\right\}$. Если у фронта $\sigma\left(L_{0}\right)$ есть ровно одна положительная псевдоинволюция, то по теореме 2.5 семейства $\left\{\mathrm{P}_{t}\right\}$ и $\left\{\mathrm{P}_{F_{t}}\right\}$ совпадают. Из этого, в частности, вытекает, что для $L \in \mathscr{L}_{1}$ непрерывное сечение $\Gamma_{\mathscr{H}(L)}$ фронта $\sigma(L)$ совпадает с минимаксным сечением, определенньм в [9]. В обшем случае семейство $\left\{\mathrm{P}_{F_{t}}\right\}$ может, в отличие от $\left\{\mathrm{P}_{t}\right\}$, не быть характеристическим.

Ограничиваясь рассмотрением семейства $\left\{F_{t}\right\}$, для которого пересечение пути $\left\{L_{F_{t}}\right\}$ с дискриминантом $\mathscr{D}$ единственно, отметим, что, согласно сказанному в $\S 4$, непрерьвное нехарактеристическое семейство псевдоинволюций может существовать лишш для перестроек типа III и XX. И для перестройки типа III, и для перестройки типа XX существуют примеры таких семейств $\left\{F_{t}\right\}$, что семейство $\left\{\mathrm{P}_{F_{t}}\right\}$ нехарактеристично. (Более того, изучая перестройки комплексов Морса для двупараметрических семейств функщий, можно показать, например, что при $\mathbb{E}=\mathbb{Z} / 2 \mathbb{Z}$ для перестройки $\mathrm{III}_{3}$ (см. п. 3.13) семейство $\left\{\mathrm{P}_{F_{t}}\right\}$ не может быть характеристическим и, следовательно, никогда не совпадает с семейством $\left\{\mathrm{P}_{t}\right\}$.) Возможная нехарактеристичность семейства псевдоинволюций $\left\{\mathrm{P}_{F_{t}}\right\}$ не позволяет без проведения дополнительных рассуждений заменить теорему 2.5 результатами теории производящих семейств в доказательстве гипотез Арнольда.

Из свойств производяших семейств можно вывести, что для семейства $\left\{F_{t}\right\}$, которое определяет петлю $\left\{L_{t}\right\}$, стягиваемую в $\mathscr{L}$, справедливо равенство $\mathrm{P}_{F_{1}}=\mathrm{P}_{F_{0}}$. В $\S 4$ было показано, что для семейства $\left\{\mathrm{P}_{t}\right\}$ равенство $\mathrm{P}_{1}=\mathrm{P}_{0}$, вообше говоря, не вьполняется.

Отметим также, что два различных семейства $\left\{F_{t}\right\}$ и $\left\{\widetilde{F}_{t}\right\}$, задающие одно и то же семейство лежандровых зацеплений $\left\{L_{t}\right\}$, могут определять разные семейства псевдоинволюций $\left\{\mathrm{P}_{F_{t}}\right\},\left\{\mathrm{P}_{\widetilde{F}_{t}}\right\}$, даже если $\mathrm{P}_{F_{0}}=\mathrm{P}_{\widetilde{F}_{0}}$.

\section{СПИСОК ЛИТЕРАТУРЫ}

[1] V. I. Arnol'd. Topological Invariants of Plane Curves and Caustics. Princeton, RI: Amer. Math. Soc., 1994. (Univ. Lecture Ser. V. 5.)

[2] В.И. Арнольд. Инварианты и перестройки фронтов на плоскости // Труды МИАН. 1995. T. 209. C. $14-64$.

[3] В.И. Арнольд. Топологические проблемы теории распространения волн // УМН. 1996. T. 51. № 1. C. 3-50.

[4] В.И. Арнольд. Что такое математика? М.: МЦНМО, 2002.

[5] В.И. Арнольд, А. Б. Гивенталь. Симплектическая геометрия // Итоги науки и техники. Сер. Соврем. проблемы матем. Фундам. направления. Т. 4. М.: ВИНИТИ, 1985. C. $5-139$.

[6] S.A. Barannikov. The framed Morse complex and its invariats // Adv. Soviet Math, 1994. V. 21. P. 93-115.

[7] W. Blaschke. Kreis und Kugel. Berlin: de Gruyter, 1956. (Рус. пер.: В. Бляшке. Круг и шар. M.: Наука, 1967.)

[8] J. Cerf. Stratification naturalle des espaces de fonctions différentiables réeles et le théorème de la pseudo-isotopie // Inst. Hautes Études Sci. Publ. Math. 1970. V. 39. P. 5-173.

[9] M. Chaperon. On generating families // The Floer Memorial Volume / ed. H. Hofer et al. Basel: Birkhäuser, 1995. P. 283-296. (Progr. Math. V. 133.)

[10] Ю. В. Чеканов. Критические точки квазифункций и производящие семейства лежандровых многообразий // Функц. анализ и его прил. 1996. Т. 30. № 2. С. 56-69.

[11] Yu. V. Chekanov. Differential algebra of Legendrian links // Invent. Math. 2002. V. 150. №3. P. 441-483. 
[12] Я. М. Элиашберг. Теорема о структуре волновых фронтов и ее применения в симплектической топологии // Функц. анализ и его прил. 1987. Т. 21. № 3. С. 65-72.

[13] Y. Eliashberg, M. Gromov. Lagrangian intersections theory: finite-dimensional approach // Geometry of Differential Equations. Providence, RI: Amer. Math. Soc., 1998. P. 27-118. (Amer. Math. Soc. Transl. Ser. 2. V. 186.)

[14] M. Entov. On the necessity of Legendrian fold singularities // Internat. Math. Res. Notices. 1998. V. 20. P. 1055-1077.

[15] E. Ferrand, P. E. Pushkar'. Non-cancellation of cusps on wave fronts // C. R. Acad. Sci. Paris Sér. I Math. 1998. V. 327. № 9. P. 827-831.

[16] D. Fuchs. Chekanov-Eliashberg invariant of Legendrian knots: existence of augmentations // J. Geom. Phys. 2003. V. 47. № 1. P. 43-65.

[17] D. Fuchs, S. Tabachnikov. Invariants of Legendrian and transverse knots in the standard contact space // Topology. 1997. V. 36. № 5. P. 1025-1053.

[18] A. Hatcher, J. Wagoner. Pseudo-isotopies of compact manifolds // Astérisque. 1973. V. 6. P. 3-274.

[19] A. Hurwitz. Über die Fourierschen Konstanten integrierbarer Funktionen // Math. Ann. 1903. V. 57. P. 425-446.

[20] S. Mukhopadhyaya. New methods in the geometry of a plane arc. I: Cyclic and sextactic points // Bull. Calcutta Math. Soc. 1909. V. 1. P. 31-37.

[21] П. Е. Пушкарь. Обобщение теоремы Чеканова. Диаметры иммерсированных многообразий и волновых фронтов // Труды МИАН. 1998. Т. 221. С. 289-304.

[22] M. Schwarz. Morse homology. Basel: Birkhäuser, 1993. (Progr. Math. V. 111.)

[23] В. Д. Седых. Теорема о четьрех вершинах выпуклой пространственной кривой // Функц. анализ и его прил. 1992. Т. 26. № 1. С. 35-41.

[24] D. Théret. A complete proof of Viterbo's uniqueness theorem on generating functions // Topology Appl. 1999. V. 96. № 3. P. 249-266.

[25] C. Viterbo. Symplectic topology as the geometry of generating functions // Math. Ann. 1992. V. 292. № 4. P. 685-710.

Московский государственньй университет им. М.В.Ломоносова;

Поступила в редакцию Московский центр непрерьвного математического образования 20.05 .2004

E-mail: pushkar@mccme.ru 